\title{
Verification and Validation Technologies for Gas Dynamic Simulations
}

\author{
A.L. Zheleznyakova \\ A.Yu. Ishlinsky Institute for Problems in Mechanics Russian Academy of Sciences, \\ 119526, Moscow, Russia \\ a.zheleznyakova@mail.ru
}

\begin{abstract}
This paper presents a review of the literature on terminology, methodology and technology of verification and validation processes related to the field of computational gas dynamics. The interdisciplinarity of the used approaches that are developed by the efforts of specialists in various branches of knowledge such as operations research, statistical analysis, and computational physics is highlighted. Such fundamental problems as comprehension of the verification procedure features for computational code and solution; comprehension of the validation process specificity for model and computational solution, identification of sources of errors and uncertainty, definition of the role of validation in predictive modeling are discussed. The basic principles of the validation based on identification and quantification of errors in the computer simulation model and numerical solutions obtained using this model are formulated. Particular attention is paid to methods for estimating the accuracy of calculations. The importance of computational software testing in the framework of verification is highlighted. The validation strategy that involves a hierarchical representation of the complex interrelated physical processes occurring in the simulated engineering system is described. Approaches to planning and conducting validation experiments are presented. Methods for estimating the experimental error, random and systematic errors are described. The necessity of using nondeterministic calculations in the validation activity to take into account the factor of experimental uncertainties in the numerical simulation process is substantiated.

Some examples of the application of the described methods are given. Verification and validation of a two-dimensional model for inviscid perfect compressible medium are performed. This model occupies the lower level in the hierarchy of integrated models of complex physicochemical processes which are developed in IPMech RAS for solving coupled problems of thermogasdynamics.
\end{abstract}

Keywords: verification, validation, computational gas dynamics, computational aerodynamics, numerical simulation, software systems.

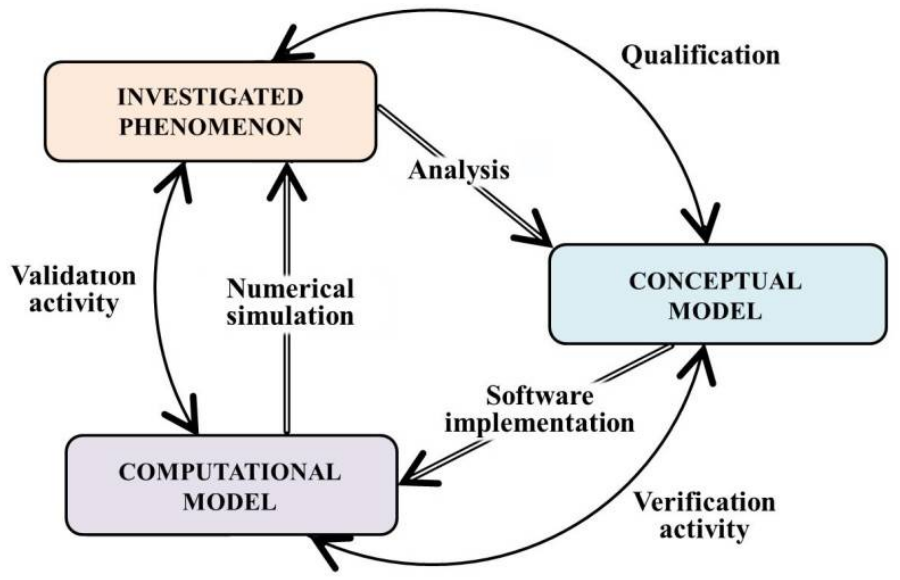

The significance of verification and validation activities at all stages of the gas dynamic simulation 


\title{
Технологии верификации и валидации в численном газодинамическом моделировании
}

\author{
А.Л. Железнякова \\ Институт проблем механики им. А.Ю. Ишлинского Российской академии наук, \\ 119526, Москва, проспект Вернадского, 101-1 \\ a.zheleznyakova@mail.ru
}

\begin{abstract}
Аннотация
В работе представлен обзор литературы, посвященный вопросам терминологии, методологии и технологии процессов верификации и валидации применительно к области вычислительной газовой динамики. Подчеркивается междисциплинарность используемых подходов, которые развиваются усилиями специалистов различных отраслей знания - исследования операций, статистического анализа, вычислительной физики. Обсуждаются такие фундаментальные проблемы, как раскрытие особенностей процедур верификации программного кода и численного решения, специфики процессов валидации модели и численного решения; выявление источников неопределенностей и ошибок; определение роли валидации в предсказательном моделировании. Формулируются основные принципы верификации, которые заключаются в выявлении и количественной оценке погрешности компьютерной модели и получаемых с ее помощью численных решений. Особое внимание уделяется методам оценки точности вычислений, подчеркивается важность тестирования программного обеспечения в рамках верификации. Излагается стратегия валидации, предполагающая иерархическое представление сложных взаимосвязанных физических процессов, протекающих в моделируемой инженерной системе. Приводятся подходы к планированию и проведению валидационных экспериментов. Описываются методы оценки экспериментальной погрешности, случайных и систематических ошибок. Обосновывается необходимость использования недетерминированных расчетов в процессе валидации для учета фактора экспериментальных неопределенностей в численном моделировании.

Приводятся примеры использования изложенных методик. Выполняется верификация и валидация двумерной модели невязкой совершенной сжимаемой среды, занимающей нижний уровень в иерархии интегрированных моделей сложных физико-химических процессов, которые разрабатываются в ИПМех РАН для решения сопряженных задач термогазодинамики.
\end{abstract}

Ключевые слова: верификация, валидация, вычислительная газовая динамика, вычислительная аэродинамика, математическое моделирование, программные комплексы.

\section{1. Введение}

Активное становление и развитие вычислительной газовой динамики (CFD) как прикладной дисциплины, в частности, при решении современных задач освоения высоких скоростей в авиастроении, стимулирует установление строгих стандартов определения степени достоверности и области применимости создаваемых компьютерных моделей. Это особенно актуально при рассмотрении пространственных течений, когда используются не полностью адекватные модели, а для их численного моделирования применяются различные допущения [1].

При современном уровне развития технологий автоматизированного проектирования полный цикл разработки новых образцов аэрокосмической техники, от создания концепции 
до летных испытаний прототипов не должен превышать трех, четырех лет. В условиях сжатых сроков этапы разработки и производства выполняются практически одновременно. При таком подходе принимать максимально эффективные инженерные решения необходимо на самой начальной стадии. Первая фаза проектирования, в ходе которой необходимо быстро сформировать технический облик изделия, выполняется главным образом на основе численного анализа. На данном этапе проводится лишь ограниченная серия натурных испытаний, необходимых для валидации компьютерных моделей. При этом от результатов, полученных методами численного моделирования, требуется предельная точность.

После того, как конфигурация изделия полностью определена, начинается фаза полномасштабных наземных и летных испытаний. На данном этапе численное моделирование может эффективно применяться для экстраполяции результатов наземных экспериментов на реальные условия высокоскоростного полета в атмосфере, численного экспресс-анализа для выбора оптимальной траектории, быстрой интерпретации экспериментальных данных.

Сочетание классических инженерных подходов и современных вычислительных технологий на всех этапах разработки перспективных изделий аэрокосмической техники позволяет повысить эффективность принятия важных конструкторских решений и снизить возможные риски.

Использование так называемых виртуальных прототипов, отчасти заменяющих натурные модели, позволяет решить множество практических задач в процессе проектирования сложных инженерных систем авиационной промышленности. Создание виртуального прототипа представляет собой сложный многостадийный процесс, включающий построение компьютерной модели поверхности, поверхностных и объемных сеточных моделей, численное решение уравнений механики сплошной среды. Последний этап - получение полного набора газодинамических данных для множества вариантов компоновок и конструкций отдельных элементов изделия в широком диапазоне условий эксплуатации [2] и визуализация результатов.

Наиболее широко для моделирования пространственного обтекания применяется осредненная по Рейнольдсу система уравнений Навье-Стокса (RANS), замыкание которой основано на использовании полуэмпирических моделей турбулентности. Подобные модели содержат различное число эмпирических констант, справедливых при определенных условиях. За более чем полувековой период развития моделей турбулентности, опыт их применения при решении различных задач показал, что та или иная модель, как правило, позволяет получить приемлемые результаты только для ограниченного класса течений. В качестве примера можно привести популярные $\mathrm{k}-\omega[3,4]$ и k- $\varepsilon$ [5] модели турбулентности в своих классических реализациях. Если первая модель корректно работает при описании пристеночной области, то вторая демонстрирует хорошие результаты при расчете свободных сдвиговых течений в отсутствии влияния стенок. Наиболее успешными для инженерных приложений считаются комбинированные подходы [6], сочетающие устойчивость и точность стандартной k$\omega$ модели в пристеночных областях с ошибкоустойчивостью и экономичностью k- $\varepsilon$ модели на удалении от стенок. Гибридный метод реализуется посредством плавного перехода от одной модели к другой, в зависимости от расстояния до обтекаемой поверхности.

Недостатки технологии, основанной на решении уравнений Рейнольдса, привели к созданию большого количества моделей турбулентности: алгебраических, дифференциальных (с различным числом дифференциальных уравнений), моделей рейнольдсовых напряжений, однако ни один из предложенных методов не обладает достаточной универсальностью. В настоящее время также активно развиваются метод прямого численного моделирования (DNS), метод моделирования с выделением крупных вихрей (LES) и др. Все подходы объединяет проблема подтверждения их достоверности при решении конкретных задач пространственного обтекания. Верификация и валидация являются основными инструментами оценки точности, надежности и достоверности вычислительных моделей, а также корректно- 
сти их программных реализаций [7-11]. Поэтому серьезное развитие концепций верификации и валидации [7-11] произошло во многом благодаря усилиям специалистов в области вычислительной газовой динамики.

Примеры тщательной верификации и валидации численных моделей и методов в рамках расчета внешних и внутренних пространственных течений, реализующихся при определенных условиях, представлены в работах $[1,7,12,13]$.

До недавнего времени национальные стандарты Российской Федерации по верификации и валидации численных методов, научного программного обеспечения и результатов моделирования $[14,15]$ разрабатывались преимущественно на базе официального перевода международных аналогов $[16,17]$, основанных на зарубежном опыте. Последние несколько лет Федеральным агентством по техническому регулированию и метрологии стали разрабатываться отечественные стандарты в области численного моделирования газодинамических течений $[18,19]$. Данные документы включают исчерпывающие постановки тестовых задач с точными аналитическими решениями или условно эталонными численными решениями, покрывающими широкий спектр течений с различными граничными и начальными условиями.

В настоящее время наблюдается стремительное развитие компьютерных технологий и совершенствование вычислительных методов, что способствует укреплению взаимодействия и росту доверия экспериментаторов к результатам математического моделирования. В обоих сообществах активно обсуждаются вопросы развития баз данных, содержащих тестовые случаи сопоставления численных решений с экспериментальными данными.

Настоящее исследование было инициировано необходимостью оптимизации и систематизации деятельности по валидации и верификации компьютерных кодов аэротермодинамики высокоскоростных летательных аппаратов, которые разрабатываются расчетно-теоретической группой ИПМех РАН в течение многих лет [2, 20-29]. Серия расчетных программ реализует иерархическую структуру компьютерных моделей различной сложности. При этом степень упрощенности каждой модели строго очерчивает круг ее применимости. Для математического описания различных течений газовой среды на структурированных и нерегулярных сетках используются: двух- и трехмерные уравнения Эйлера / Навье-Стокса, уравнения Рейнольдса с различными моделями турбулентного смешения, интегрированные модели физической и химической кинетики, модели переноса излучения и др.

В последнем разделе приводятся примеры использования изложенных ниже технологий верификации и валидации применительно к одной из подмоделей - 2D версии программы, реализующей расчет двумерного течения невязкого сжимаемого газа. Для данного класса задач могут быть получены аналитические решения, которые необходимы для верификации тестируемого программного обеспечения, оценки возможностей численных схем в выявлении свойств реальных потоков [19]. Кроме этого, для невязких сжимаемых течений получено большое число условно эталонных численных решений высокой точности, которые были многократно воспроизведены различными авторами [30-35] с использованием разнообразных численных схем [19].

\section{2. Терминология верификации и валидации}

Фундамент для современных концепций верификации и валидации заложили известные философы науки двадцатого века Поппер К. [36, 37] и Карнап Р. [38]. Обширное исследование по истории и философии науки с точки зрения проблем верификации и валидации проведено в [39]. Достаточно подробные библиографические обзоры по данной тематике опубликованы в [40-42].

Первая техническая дисциплина, в которой начали затрагиваться вопросы верификации и валидации - исследование операций [43]. Эта наука занимается разработкой и применением методов математического моделирования для нахождения и обоснования оптимальных решений в различных областях человеческой деятельности [27]. Авторы научно-прикладных исследований [30-35, 44-120] внесли существенный вклад в решение задач верификации и 
валидации вычислительных моделей. В приведенных работах получены численные решения высокой точности или аналитические решения, которые широко используются для верификации. В некоторых публикациях предложены специальные численные методы, позволяющие увеличить точность результатов, например, метод экстраполяции Л. Ричардсона [52]. В последние годы такие эталонные исследования стали упоминаться как верификационные “бенчмарки".

Значительная часть работ [44-120] посвящена созданию методологии валидации и постановке так называемых валидационных экспериментов. Последние существенно отличаются от классических опытов и проводятся с целью проверки адекватности математической модели. Такие эксперименты выполнятся в четко определенных условиях, требуют точного контроля и строгой оценки неопределенности измерений. Для проведения валидирующих экспериментов необходимо более тесное сотрудничество между специалистами-вычислителями и экспериментаторами.

Как отмечалось выше, численные реализации представляют собой аппроксимацию реальных физических процессов, а, следовательно, являются только приближением к действительности. Они нуждаются в оценке степени достоверности путем верификации и валидации.

Верификация - это процесс определения степени полноты, адекватности и уровня точности численной реализации принятой концептуальной модели. Проблема верификации математических моделей газодинамических явлений затрагивает вопросы сплошной среды, дискретной математики и верности сложных логических структур (компьютерных кодов).

Задача валидации - установить, в какой мере адекватность формальных теоретических конструкций (математических моделей) может быть проверена данными физических наблюдений. Валидация позволяет определить, насколько точно выбранная концептуальная модель описывает исследуемое физическое явление путем сопоставления численных и экспериментальных данных [121]. Валидационный процесс должен строиться на количественной оценке расхождения опытных данных с результатами моделирования и включать обязательное определение погрешностей и неопределенностей как экспериментального, так и численного подходов. Кроме этого валидация требует проведения множества недетерминированных расчетов, для моделирования неопределенности в постановке граничных и начальных условий в эксперименте. Часто под стандартной процедурой валидации в вычислительной газовой динамике (как и в других областях) ошибочно понимают простое графическое сопоставление экспериментальных и расчетных данных. Если результаты соотносятся удовлетворительно, то полученное численное решение считается валидированным. Очевидно, что сравнение эмпирических и численных результатов на графике немногим лучше качественного сравнения. С помощью графического сопоставления невозможно количественно оценить численные ошибки или ошибки, возникающие вследствие неопределенности задания граничных и начальных условий, параметров моделирования.

Схематически процессы верификации и валидации, включающие как вычислительные, так и физические аспекты [51], представлены на рис.1. Рисунок иллюстрирует место рассматриваемых процедур в единой стратегии оценки достоверности вычислительных моделей. Достоверность моделирования подтверждается на основании оценки соответствия выбранной концептуальной модели реальному явлению и результатов сравнения с экспериментальными данными. При этом проверка адекватности математической модели подразумевает: определение порядка сходимости численных решений в сравнении с точным аналитическим решением, если оно существует, или, когда размер элементов расчетной сетки стремится к нулю; оценку чувствительности алгоритма дискретизации к изменениям граничных условий, при адаптации сетки к геометрии обтекаемой модели, и т.д.

На рис.1 отражены два типа моделей - концептуальная (математическая) и компьютерная. Концептуальная модель содержит исходные данные численного моделирования и математические уравнения, которые описывают исследуемую физическую систему, процесс или явление. 


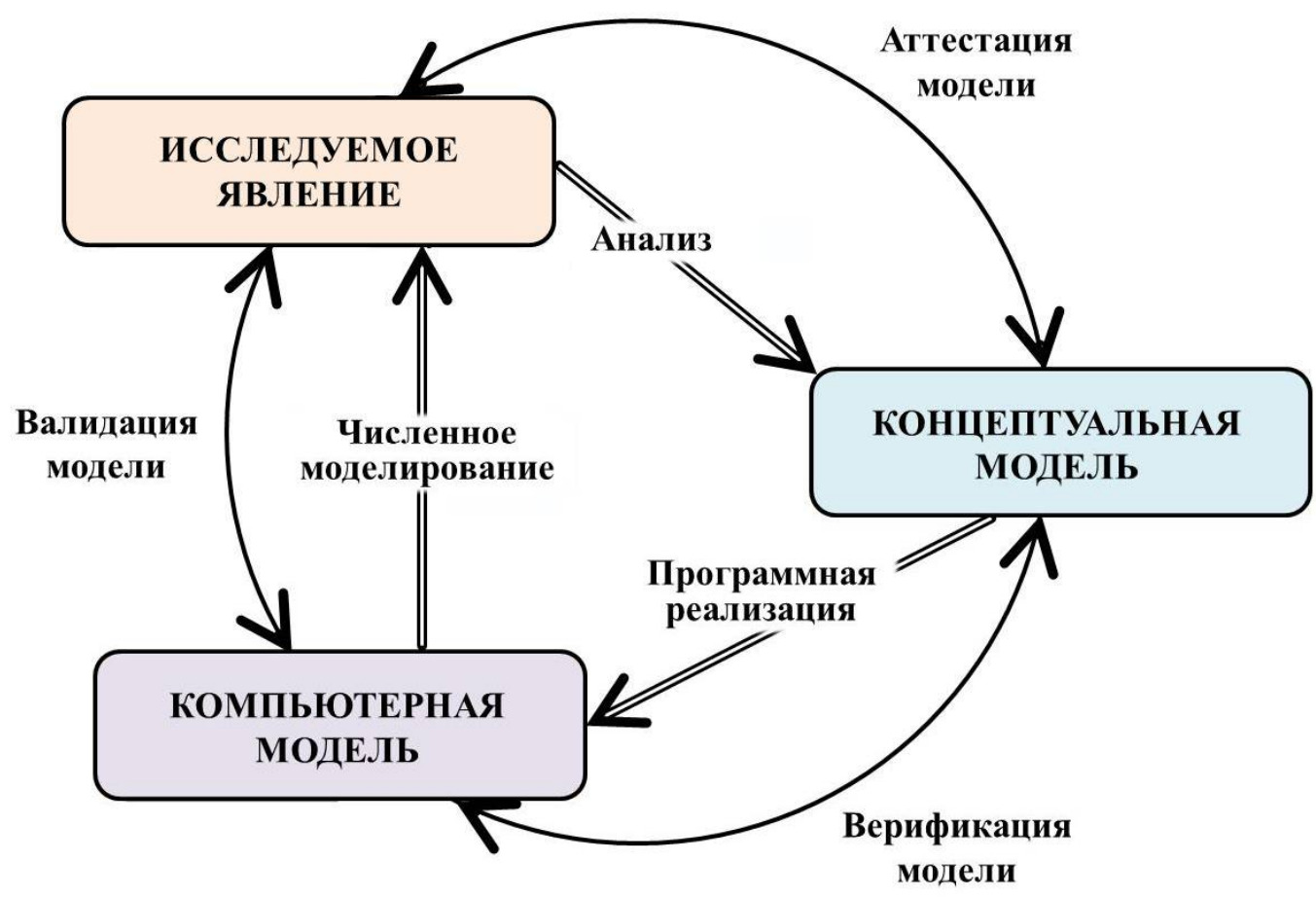

Рис. 1. Роль верификации и валидации на различных стадиях имитационного моделирования [121]

Концептуальная модель строится на основе анализа и наблюдения рассматриваемого физического объекта. В вычислительной газовой динамике концептуальная модель обычно представлена системой уравнений в частных производных, выражающих законы сохранения массы, импульса и энергии, а также начальными и граничными условиями для рассматриваемой системы. Математическая модель может быть дополнена уравнениями химической кинетики, уравнениями модели турбулентности и т.д.

Компьютерная модель представляет собой исполняемую программу (вычислительный код), реализующую принятое математическое описание исследуемой системы (концептуальную модель).

Из рис. 1 видно, что верификация устанавливает взаимосвязь между концептуальной и компьютерной моделями. Валидация, в свою очередь, определяет корреляцию между компьютерной моделью и исследуемым физическим явлением или процессом. По существу, верификация и валидация являются инструментами для оценки точности концептуальных и компьютерных моделей. Основные задачи верификационной и валидационной деятельности определение степени достоверности моделей и меры доверия к ним. Под аттестацией понимается проверка адекватности математической модели для области предполагаемого применения.

При решении сопряженных задач сверхзвуковой аэротермодинамики используемая математическая модель должна описывать множество процессов (радиационно-конвективный теплообмен, химические реакции), учитывать эффекты реального газа, влияние каталитичности поверхности и др. Принимая во внимание, что для многих инженерных приложений количественный анализ точности является обязательным, его проведение для подобных интегрированных моделей становится очень серьезной проблемой. В некоторых случаях оценка точности комплексной модели требует проведения анализа точности всех физических моделей низшего уровня, описывающих отдельные процессы.

Для преодоления перечисленных трудностей необходимо разрабатывать универсальные методы оценки качества для множества условий, в которых применение данной модели является целесообразным. 
В 1992 году комитет стандартов по вычислительной газовой динамике Американского института аэронавтики и астронавтики (AIAA) начинает проект по формулировке и стандартизации терминологии и методологии верификации и валидации численных реализаций моделей газовой динамики [122].

\section{3. Методология верификации}

Верификация вычислительного кода подразумевает подтверждение того, что программная реализация модели точно отражает принятую разработчиком концептуальную модель и соответствующее данной модели решение. В процессе верификации устанавливается, что математическая модель решена правильно с использованием методов дискретной математики, реализованных в компьютерной программе.

Ниже приводится основные методологические принципы верификации, на которые рекомендуется опираться при использовании методов конечных разностей, конечных элементов, контрольных объемов и граничных элементов [51].

Стратегия верификации заключается в идентификации и количественной оценке ошибок расчетной модели. Для количественной оценки погрешности численного решения используются высокоточные, надежные, эталонные решения (“бенчмарки”), которые могут быть как численными, так и аналитическими. Как правило, прецизионно точные решения получают для задач, где используются упрощенные модели. При этом не требуется, чтобы концептуальная модель как-то соотносилась с реальным миром, так как верификация рассматривает вопросы математики и программирования, но не физики. На рис.2 схематически показана процедура верификации, которая проводится путем сравнения численного решения с эталонным [122]. Процесс выполняется на ранних стадиях цикла разработки компьютерного кода, и повторяется при каждой последующей модификации программы. Хотя достоверность численных результатов, получаемых в процессе верификации, зависит от конкретной задачи и области предполагаемого применения кода, к точности решений предъявляются более жесткие требования, чем при валидации.

Для устойчивой, согласованной и надежной численной схемы существует пять источников ошибок в решениях: недостаточная пространственная дискретизация; недостаточная временная дискретизация; недостаточная степень сходимости в итерационной процедуре; ошибки округления, неизбежно возникающие при вычислениях на компьютере; ошибки программирования. Таким образом, задачами верификации являются: выявление и количественная оценка указанных ошибок, а также демонстрация устойчивости и надежности численной схемы. Выводы об успешности верификации должны быть четко аргументированы и строго обоснованы. Аналитический или формальный подход к оценке ошибок является неприемлемым для верификационной деятельности.

Для количественной оценки ожидаемой степени расхождения между численным и эталонным решениями (рис. 2) вычисляют как локальную погрешность численного метода, вносимую после одного шага интегрирования, так и глобальную ошибку, накопленную за определенное количество временных шагов.

Как правило, в литературе, посвященной численным методам решения задач газовой динамики, проблемам верификации предлагаемых кодов уделяется недостаточно внимания. Большая часть работ носит исследовательский характер и сосредотачивается на таких аспектах моделирования, как вычислительные алгоритмы, построение расчетных сеток, визуализация и т.д. 


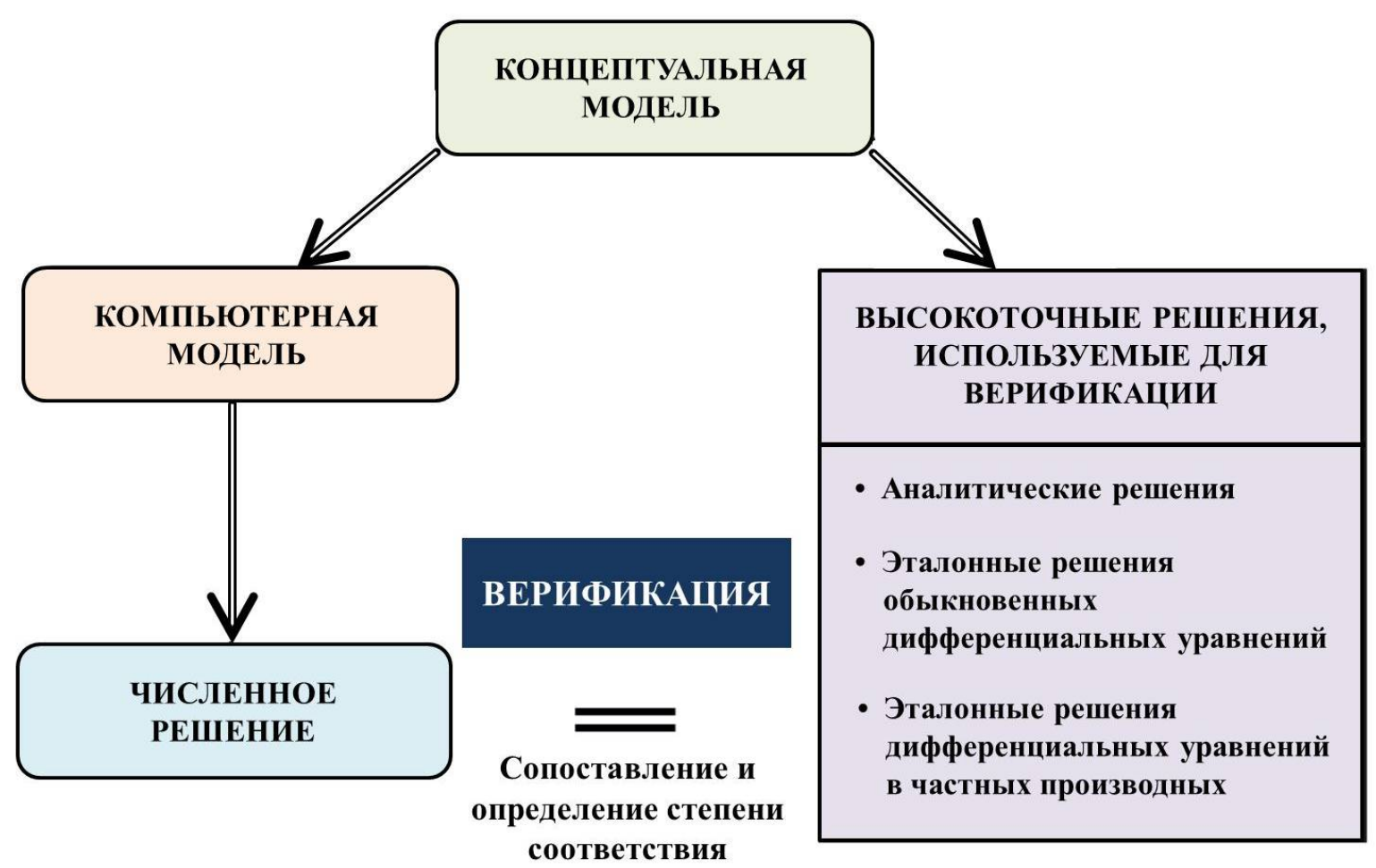

Рис. 2. Процесс верификации [122]

\section{4. Технология верификации газодинамических кодов}

Верификация - равноправный партнер валидации в единой стратегии проверки качества программных средств вычислительной аэродинамики. Для численных методов решения дифференциальных уравнений, где используются сложные схемы дискретизации, высока вероятность, что ошибка в коде будет принята за погрешность приближенных вычислений. Поэтому исследование погрешности математического моделирования проводится исключительно эмпирическим путем. В идеале необходимо продемонстрировать, что ошибка вычислений мала для всех возможных применений программного обеспечения (ПО), что является невозможным для сложных кодов. Однако поставленная цель может быть достигнута для ограниченной серии расчетов.

Основной задачей верификации научного ПО является оценка точности численных решений, полученных с использованием расчетного кода, с учетом, особенностей уравнений, лежащих в его основе. При этом необходимо доказать, что компьютерная модель сохраняет адекватность для случаев отличных от верификационных тестов. Таким образом, основным требованием при проведении верификации является обеспечение максимальной уверенности в точности новых расчетов (т.е. решение так называемой задачи оценки доверия). Прогностический анализ точности, которую будет обеспечивать вычислительная программа при решении новых задач, проводится на основе опыта предыдущего применения расчетного кода.

В процессе верификации вычислительных кодов используются следующие принципы, методы и подходы, позаимствованные из различных областей знаний:

1) Классическая теория сходимости разностных схем в приложении к уравнениям в частных производных [123-125].

2) Тестирование на основе формальных моделей [8, 126, 127].

3) Использование верификационных “бенчмарков” как стандартов для количественной оценки точности.

4) Контроль качества программного обеспечения [8]. 
Как отмечалось ранее, верификация проводится на начальных этапах жизненного цикла ПО и повторяется после любой модификации вычислительного кода. В процессе верификации необходимо решить следующие задачи:

1) Строго доказать, что компьютерная реализация полностью соответствует построенной концептуальной модели и ее решению. Это, в свою очередь, требует подтверждения того, что реализованные численные алгоритмы правильно аппроксимируют лежащие в ее основе уравнения с заданным набором начальных и граничных условий.

2) Доказать, что реализованный алгоритм обеспечивает сходимость к правильному решению этих уравнений при любых условиях, для которых вычислительный код будет применен.

Процедура верификации вычислений является одним из этапов многопланового процесса верификации компьютерного кода. Основная цель верификации вычислений - подтверждение верности и точности конкретного расчета, но даже этот промежуточный шаг может представлять значительную трудность. Например, верификация численного решения требует доказательства сходимости по сетке, что для сложных инженерных задач выполнить просто невозможно, вследствие ограниченности вычислительных ресурсов.

Наличие высокоточных результатов численного моделирования по схожей задаче может служить веским аргументом того, что сошедшееся решение верно. Однако при рассмотрении незначительно различающихся случаев данная логика может быть нарушена. Поэтому для обоснования корректности решения конкретной задачи используется метод доказательства “от противного". Подход подразумевает тщательный сбор данных об отсутствии неверных вычислений, выполненных с использованием верифицируемого кода (доказать ошибочность расчета намного проще). Это очень длительный и сложный процесс, поэтому в случае коммерческих пакетов основную работу по верификации выполняют пользователи после выхода программного продукта.

В руководстве [122] ошибка вычислений определяется как распознаваемый недостаток, возникающий на любом этапе моделирования, который не является следствием пробелов в знаниях или недопонимания сущности исследуемого явления.

Построение любой математической модели и ее реализация связаны с упрощением исходного объекта или явления и внесением погрешностей (ошибок). Последние называются погрешностями модели и относятся к неустранимым ошибкам. При переходе от математической модели к численному методу возникают погрешности, которые носят название ошибок метода. К данному классу погрешностей относятся ошибки дискретизации и усечения (обрыва). При реализации численного метода на ЭВМ возникают погрешности округления. Таким образом, основными источниками ошибок являются:

1) Погрешность математической модели, которая связана с ее несоответствием физической реальности (различные аппроксимации и допущения, такие как приближения сплошной среды, несжимаемости низкоскоростного течения и т.д.).

2) Погрешность исходных данных, принятых для расчета.

3) Погрешность метода, связанная с дискретным характером любого численного алгоритма.

4) Погрешность округления, которая связана с использованием в вычислительных машинах чисел с конечной точностью представления.

5) Ошибки программирования, ошибки компилятора.

Количественная оценка ошибок имеет первостепенное значение для процесса верификации. Под ошибкой вычислений $E$ в газовой динамике обычно понимают следующее [122]:

$$
E=u_{\text {exact }}-u_{\text {discrete }}
$$


Здесь $u_{\text {exact }}$ - точное решение системы уравнений математической модели для заданного набора начальных и граничных условий; $u_{\text {discrete }}$ - численное решение дискретной аппроксимации данных уравнений при тех же условиях, полученное с использованием конкретного кода на определенной вычислительной машине.

Численная ошибка $E$ зависит от пространственной и временной дискретизации. Дискретизация по пространству характеризуется размером шага расчетной сетки $h$. Шаг одно-, двух- или трехмерной сетки может варьироваться как по пространству, так и по времени для адаптированных к геометрическим особенностям и адаптивных динамических сеток соответственно. Временная дискретизация определяется шагом по времени $\tau$.

Пусть дискретное отображение непрерывного решения исходной системы уравнений $u_{h, \tau \rightarrow 0}$ стремится к точному решению при $h \rightarrow 0$ и $\tau \rightarrow 0$. Тогда для ошибки численного решения будет справедливо [128]

$$
E \leq\left\|u_{\text {exact }}-u_{h, \tau \rightarrow 0}\right\|+\left\|u_{h, \tau \rightarrow 0}-u_{h, \tau, I, c}\right\|<\varepsilon,
$$

где $u_{h, \tau, I, c}$ - дискретное решение, полученное с использованием данного численного алгоритма при определенных параметрах пространственной $h$ и временной дискретизации $\tau$, коэффициенте итеративной сходимости $I$, на конкретной вычислительной машине $c ; \varepsilon-$ произвольно малое число, задающее точность численного решения.

Первое слагаемое $\left\|u_{\text {exact }}-u_{h, \tau \rightarrow 0}\right\|$ в соотношении (2) это ошибка, вносимая аппроксимацией исходной системы уравнений ее дискретным аналогам (погрешность, возникающая вследствие точного решения дискретных уравнений при $h, \tau \rightarrow 0)$. Исходя из принципов построения алгоритмов для численного решения дифференциальных уравнений в частных производных, можно принять, что это слагаемое равно нулю. Однако последнее справедливо только для линейной системы с постоянными коэффициентами и согласованных, устойчивых численных схем. Для современных прикладных вычислений, при описании физики многомасштабных нелинейных процессов используются сложные нелинейные, трехмерные, нестационарные математические модели. Сложность доказательства равенства нулю первого слагаемого (2) иллюстрируется в [129] на различных вычислительных примерах.

Второе слагаемое $\left\|u_{h, \tau \rightarrow 0}-u_{h, \tau, I, c}\right\|$ в формуле (2) отлично от нуля, т.к. параметры $h, \tau$ и $I$, имеют конечные значения, а также вследствие использования компьютерной арифметики ограниченной точности, ошибок программирования в исходных кодах вычислительной программы и операционной системы.

Как отмечалось ранее, дискретная аппроксимация исходной системы уравнений в частных производных и ее последующее решение с использованием конечно-разностного или конечно-объемного подходов приводит к возникновению ошибок усечения и дискретизации [128].

Различие между решением дискретного аналога исходной системы уравнений и ее точным решением будет определяться свойствами используемой численной схемы, такими как устойчивость, согласованность и сходимость. Проверка на согласованность заключается в подстановке точного решения в конечно-разностные уравнения для каждого узла расчетной сетки, с последующим разложением в ряд Тейлора в окрестности узлового значения. При этом бесконечный ряд усекают (обрывают), заменяя усеченную часть остаточным членом. После замены дискретный аналог математической модели преобразуется в исходную систему дифференциальных уравнений (дискретная аппроксимация согласуется с исходной системой дифференциальных уравнений в частных производных) с точностью до остаточного члена. Последний принимают за погрешность усечения. Таким образом, дискретизация считается строго согласованной, если численное решение сходится к точному с нулевой ошибкой усечения при стремлении к нулю временного и пространственного шагов [128].

При установлении согласованности принимается предположение о гладкости дискретной аппроксимации, которое, строго говоря, является ошибочным для широкого спектра 
сжимаемых течений. Использование производных, определенных в узлах сетки, для вычисления ошибки усечения приводит к локальному нарушению условия сходимости и согласованности в окрестности разрывных решений (например, ударных волн, контактных разрывов). Наличие разрывов в решении дифференциальных уравнений существенно усложняет поставленную задачу.

Для согласованной схемы дискретизации (в одномерном случае) погрешность усечения оценивается как $O\left(h^{p}, \tau^{q}\right), p>0, q>0$, где $p$ и $q$ - порядок точности соответственно пространственной и временной дискретизации [128].

Количественная оценка ошибки дискретизации проводится на основании предположения о ее пропорциональной зависимости от погрешности усечения [130], справедливого при использовании достаточно хороших расчетных сеток и дополнительных упрощающих допущений (линейности и постоянства шага пространственной дискретизации). Связь между погрешностями дискретизации и усечения (при выполнении условия сходимости численного решения и достаточно малых $h$ и $\tau$ ) устанавливается следующим образом [130]:

$$
\left\|u_{\text {exact }}-u_{h, \tau}\right\|=O\left(h^{p}, \tau^{q}\right)
$$

Соотношение (3) может использоваться для локальной оценки ошибки дискретизации, если шаг сетки непостоянный.

Свойство согласованности является важнейшим для всего процесса верификации. Для дискретизации, не удовлетворяющей условию согласованности, сходимость решения будет отсутствовать. Согласованность - необходимое условие, при котором первое слагаемое в соотношении (2) может обратиться в нуль. Однако данное условие не является достаточным и не обеспечивает гарантированную сходимость численного решения к точному решению исходной системы дифференциальных уравнений в частных производных, даже если оно единственное. Дополнительным условием является устойчивость дискретизации.

Только одна общая теорема - теорема эквивалентности Лакса (ее строгое доказательство приводится в [131]) подтверждает, что устойчивые согласованные схемы действительно сходятся. Теорема эквивалентности Лакса устанавливает эквивалентность устойчивости и сходимости для линейных систем. Хотя теорема не может быть непосредственно применена к нелинейным уравнениям, соотношение (3), полученное в предположении линейности, может также эффективно использоваться для оценки локальной ошибки дискретизации для нелинейных задач.

Для случая нелинейных систем широко применяют анализ устойчивости по фон Нейману [132]. Однако эта технология также основывается на допущении о постоянстве шага пространственной дискретизации. В работе [128] подробно рассматриваются различные элементы метода анализа устойчивости нелинейных систем, основанного на понятии монотонности и концепции ограниченности полной вариации.

Количественный анализ погрешности дискретизации позволяет ответить на следующие ключевые вопросы верификации:

1) Сходится ли численное решение к точному, при уменьшении пространственного шага?

2) Каков истинный (эффективный) порядок точности пространственной $p$ и временной $q$ дискретизации, наблюдаемый в расчетах?

3) Какова фактическая ошибка дискретизации, наблюдаемая в конкретном расчете на сетке с конечным шагом?

Выше были рассмотрены методы анализа погрешности вычислений, которые основаны на априорной информации. В рамках данного подхода оценка проводится без прямого наблюдения за ходом выполнения реализованных алгоритмов и в отсутствие результатов численного моделирования, например, с использованием соотношения (3). Априорный ана- 
лиз погрешностей базируется на классическом численном анализе дифференциальных уравнений, лежащих в основе газовой динамики [128, 130, 133, 134], и является важнейшим элементом верификации.

Апостериорную оценку погрешности дискретизации проводят на основе сопоставления расчетных данных, полученных при различных значениях шага дискретизации по времени и пространству. Учитывая, что пространственная и временная сходимость решения имеют асимптотический характер, с уверенностью можно постулировать лишь следующее:

$$
\left\|u^{r 1}-u^{r 2}\right\| \leq \varepsilon
$$

где $r 1$ - степень пространственного и временного разрешения; $r 2$ - более высокая степень разрешения; $\varepsilon$ - требуемая точность решения. Для анализа сходимости по сетке, имеющей монотонный характер (различают также осциллирующую сходимость и отсутствие сходимости) используется обобщенный метод экстраполяции Ричардсона $[51,135]$, позволяющий оценить точность решения на сетке с шагом $h$, если имеется несколько решений задачи на различных сетках: $h_{1}=h, h_{2}=r h, h_{3}=r^{2} h$, (обычно принимают $r=0.5$ ).

Остановимся подробнее на вопросе сеточной сходимости. Для простоты рассмотрим стационарную одномерную задачу. Область, в которой ищется решение, разбивается однородной сеткой с постоянным шагом. Для применения экстраполяции Ричардсона необходимо сделать ряд допущений:

1) $u$ - гладкое решение (условие обеспечивает существование всех необходимых производных для применения разложения в ряд Тейлора).

2) Схема дискретизации по пространству имеет формальный порядок сходимости $p$, известный априори. Кроме этого, компьютерный код на практике демонстрирует сходимость порядка $p$.

С учетом сделанных предположений, точное решение представимо в виде [135]

$$
u_{\text {exact }}=u_{h}+\alpha h^{p}+O\left(h^{p+1}\right),
$$

где $\alpha$ - константа. Обозначения остальных величин приводились ранее. Если $u_{h}$ и $p$ известны, то для расчета двух неизвестных величин в соотношении (5) требуется два численных решения, полученных на сетках различной подробности (характеризующихся шагом $h_{1}$ и $h_{2}$ ). Таким образом, оценка ошибки дискретизации может быть выполнена на основе двух численных решений (предположим, что $h_{2} / h_{1}<1$ )

$$
\begin{aligned}
& u_{\text {exact }}=u_{h 1}+\alpha h_{1}^{p}+O\left(h_{1}^{p+1}\right), \\
& u_{\text {exact }}=u_{h 2}+\alpha h_{2}^{p}+O\left(h_{2}^{p+1}\right)
\end{aligned}
$$

Отсюда находим

$$
\begin{gathered}
\alpha=\left[\frac{u_{h 1}-u_{h 2}}{h_{2}^{p}-h_{1}^{p}}\right]+O\left(h_{1}^{p+1}\right)+O\left(h_{2}^{p+1}\right) \\
u_{\text {exact }}=\left[\frac{h_{2}^{p} \cdot u_{h 1}-h_{1}^{p} \cdot u_{h 2}}{h_{2}^{p}-h_{1}^{p}}\right]+O\left(h_{1}^{p+1}\right)+O\left(h_{2}^{p+1}\right)
\end{gathered}
$$

Соотношение в скобках представляет собой экстраполяцию численного решения к точному. Достоверность экстраполяции будет зависеть от того, сколько решений $\left(u_{h 1}, u_{h 2}, u_{h 3}, \ldots\right)$ использовалось.

Таким образом, апостериорная ошибка дискретизации для численного решения (например $u_{h 1}$ ) может быть определена как 


$$
\left\|u_{\text {exact }}-u_{h 1}\right\| \cong\left|\left[\frac{u_{h 2}-u_{h 1}}{h_{2}^{p}-h_{1}^{p}}\right] h_{1}^{p}\right|+O\left(h_{1}^{p+1}\right)+O\left(h_{2}^{p+1}\right)
$$

Соотношение (9) может быть расширено на двумерные и трехмерные нестационарные задачи.

Рассмотренный метод экстраполяции Ричардсона имеет существенные недостатки. Вопервых, высокая ресурсоемкость и необходимость значительных временных затрат существенно ограничивают применение подхода. Во-вторых, если численный метод формально имеет порядок точности $p$, то совсем не обязательно, что при практическом использовании его программной реализации полученное решение будет того же порядка.

Наличие разрывов (ударные волны, контактные разрывы) существенно усложняют процесс верификации и, естественно, влияют на процедуру экстраполяции. Проблема в том, что производные высокого порядка, которые не учитываются при разложении в ряд Тейлора в процессе дискретизации, нельзя считать малыми в окрестности разрывов. В таких случаях применяют специальные математические преобразования, позволяющие исключить особенности. Если это невозможно (например, для сложных сверхзвуковых течений, характеризующихся наличием ударных волн и разрывов), то простого уменьшения пространственного и временного шага будет недостаточно для достижения хорошего сеточного разрешения. Для подобных задач дополнительно проводят анализ влияния разрывов на сеточное решение в остальной области.

В работе [136] подробно рассмотрен вопрос о порядке сходимости численных схем с динамической адаптацией, применяющихся для решения двумерных задач газовой динамики без явного выделения разрывов. Результаты исследования показали, что для всех схем сквозного счета на фронтах ударных волн порядок аппроксимации снижается до первого, независимо от точности численного метода.

Допущение о гладкости решения, лежащее в основе экстраполяции Ричардсона, удалось ослабить авторам работы [137] при моделировании сверхзвукового обтекания сферически затупленных конусов. В исследовании подчеркивается важность учета членов более высокого порядка при применении экстраполяции Ричардсона для вычисления погрешности дискретизации. Предложенный подход предполагает использование трех численных решений, полученных на сетках с исходным и увеличенным разрешением.

$$
\begin{gathered}
u_{\text {exact }}=u_{h 1}-g_{1} h_{1}-g_{2} h_{1}^{2}, \\
g_{1}=\frac{u_{h 3}-u_{h 2}-r^{2}\left(r^{2}-1\right) u_{h 2} h_{1}^{2}}{r(r-1) h_{1}}, \quad g_{2}=\frac{u_{h 3}-u_{h 2}-r\left(u_{h 2}-u_{h 1}\right)}{r(r-1)\left(r^{2}-1\right) h_{1}^{2}}
\end{gathered}
$$

Вместо известного априори формального порядка дискретизации (см. второе допущение метода Ричардсона) численной схемы, целесообразней использовать его действительное значение, наблюдаемое в ходе экстраполяции [51]. В работе [138] предлагается следующая зависимость для определения действительного порядка дискретизации, включающая численные результаты, полученные на трех сетках различной подробности при достижении асимптотической сходимости

$$
\begin{gathered}
\tilde{p}=\ln \left(\frac{u_{h 3}-u_{h 2}}{u_{h 2}-u_{h 1}}\right) / \ln (r), \\
r=\frac{h_{3}}{h_{2}}=\frac{h_{2}}{h_{1}}
\end{gathered}
$$


Оценка апостериорных ошибок имеет первостепенное значение для конечно-элементных методов, где широко применяется адаптация расчетных сеток (так называемая $h$-адаптация), а также используется подстройка порядка аппроксимации численной схемы $p$-адаптация).

Современные задачи численного моделирования сложных инженерных систем решаются на пределе доступных на сегодняшний день вычислительных ресурсов. Если дальнейшее сгущение сетки не представляется возможным для получения двух уровней уточнения (9), сеточное разрешение, напротив, уменьшают [51]. Следует отметить, что огрубление сетки недопустимо, если это нарушит асимптотическую устойчивость дискретного решения. Для моделирования сжимаемых течений разрешение сетки должно быть достаточным для того, чтобы на область выделенного разрыва приходилось, например, не менее четырех расчетных узлов. В этом случае огрубление сетки нарушит ключевые принципы, лежащие в основе экстраполяции. Следовательно, необходимо разрабатывать методы оценки апостериорных ошибок на основе результатов, полученных на единственной сетке.

Отметим, что для сложных инженерных приложений хорошее пространственное разрешение не может быть достигнуто одновременно во всей моделируемой области. Поэтому, возникает необходимость в дополнительном анализе влияния ошибки дискретизации, возникающей в недостаточно разрешенных областях, на соответствующую погрешность в зонах с хорошим пространственным разрешением.

\section{5. Верификационное тестирование}

Тестирование - один из ключевых элементов многопланового процесса контроля качества компьютерного кода и важнейший этап верификации вычислительных моделей газовой динамики.

В работе [139] приводятся результаты всестороннего анализа качества более чем сотни кодов научного программного обеспечения, при этом автор обрисовывает весьма мрачную картину. Тестируемые программные продукты поставлялись компаниями, правительственными учреждениями и университетами различных стран мира. Коды предназначались для применения в самых различных сферах, затрагивающих графику, ядерные технологии, машиностроение, химическую и аэрокосмическую отрасли, строительство, связь, базы данных, медицинские системы. Многие предназначались для критически важных вычислений и достигли высокого уровня зрелости (регулярно применялись своим кругом пользователей и были одобрены для применения).

Важнейшие выводы, полученные автором в ходе тестирования, заключаются в следующем:

1) В исходных текстах на языках Си и Фортран в среднем присутствовало соответственно 8 и 12 серьезных ошибок на 1000 исполняемых строк кода. Очевидно, что эти ошибки не приводили к сбою работы программы, однако оказывали существенное влияние на конечный результат. Ошибки, не нарушающее ход выполнения алгоритмов, однако приводящие к умеренно неправильным результатам, представляют наибольшую опасность - их почти невозможно обнаружить.

2) Результаты, которые были получены при идентичных входных данных с использованием девяти программных реализаций одного и того же математического алгоритма, написанных на одном языке, согласовывались только в одной или двух значащих цифрах.

3) Программные реализации, которые предназначены для решения одного круга задач, написанные на Фортран, в среднем, в 2.5 раза длиннее, чем их аналоги на языке Си. Функции, запрограммированные на Фортран, имели больше параметров и аргументов, чем соответствующие программные единицы на языке Си. Хотя количество ошибок с увеличением длины кода возрастает, ни один язык программирования, используемый в разработке научного кода, не застрахован от их появления. 
4) Проблемы, выявленные в ходе всестороннего тестирования [139], свойственны конкретным подходам, которые используются для разработки вычислительных кодов, и не зависят от деталей их программной реализации и языка программирования.

По результатам проведенных исследований автор [139] дает неутешительное заключение (с которым многие могут не согласиться), что степень доверия большинства программных продуктов не выше, чем у неподтвержденного физического эксперимента.

В настоящем исследовании под тестированием (или формальным тестированием) будет пониматься процесс выявления ошибок в компьютерном коде в рамках верификационной деятельности с целью отладки и контроля качества научного программного обеспечения с соблюдением требований стандартов и практических руководств [140-143]. Тестирование, в ходе которого основное внимание уделяется корректности программной реализации с точки зрения технологии программирования (Software Quality Testing), является частью стратегии по обеспечению качества программного обеспечения.

Формальное тестирование вычислительных кодов газовой динамики не является общепринятой практикой по нескольким причинам:

1) Формальный процесс тестирования программного обеспечения, в первую очередь, сосредоточен на оценке программной реализации, тогда как в вычислительной газовой динамике, при корректной реализации, акцент традиционно ставится на численной эффективности.

2) Создатели газодинамических кодов тратят основные усилия на разработку математической модели, численных методов и эффективных алгоритмов, однако, по сравнению с инженерами-программистами, уделяют меньше внимания технической проверке и детальной проработке своих кодов.

3) CFD-коды не относятся к критически важным приложениям по обеспечению безопасности, поэтому предполагаемый риск, обусловленный неправильным функционированием CFD-кодов, не так значителен.

Современные методы тестирования не позволяют однозначно и полностью устранить все ошибки и дефекты, а также установить корректность функционирования научного программного обеспечения. По этой причине производители программных продуктов выпускают так называемые "патчи" (автоматизированные отдельно поставляемые программные средства, используемые для устранения проблем в программном обеспечении или изменения его функциональности), пакеты обновлений, обновленные версии программ.

Как неоднократно отмечалось, в вычислительной газовой динамике используется комплексный подход к верификации, который включает разнообразные виды верификационной деятельности. Комплекс проверочных мероприятий, включающих инженерию качества вычислительных кодов (Software Quality Engineering) [140-143], которые составляют процедуру верификации, представлен на рис.3. При этом верификация кода и верификация вычислений выделяются в иерархической структуре процесса как отдельные ветви.

При оценке качества программного обеспечения проводится два класса испытаний, различающихся по критерию запуска программы - статическое и динамическое тестирование $[126,127,144]$.

При статическом тестировании программный код не выполняется, а само тестирование может быть как ручным, так и автоматизированным. Статическое тестирование начинается на ранних этапах жизненного цикла программного обеспечения и подразумевает вычитку синтаксиса программного кода, проверку его структуры, последовательности выполнения алгоритмов, а также установление соответствия требованиям проектной документации и функциональной спецификации. Статическое тестирование включает в себя следующие виды деятельности [145]:

1) Инспекция - анализ, основанный на визуальной проверке текста программы с целью поиска ошибок, нарушений стандартов разработки и несоответствий документации $[146,147]$. 
2) Аудит - независимая оценка программного обеспечения с целью установления соответствия стандартам, рекомендациям, спецификациям и документам, которые определяют: форму и содержание программного продукта; процесс, согласно которому программный продукт будет произведен; как будет измеряться соответствие стандартам или рекомендациям [147].

3) Анализ потока данных - вид статического анализа, в процессе которого: определяются зависимости между входными и выходными переменными; составляется полный список значений, используемых в программе; определяются условия, влияющие на значения переменных.

\section{Верификация в вычислительной газовой динамике}

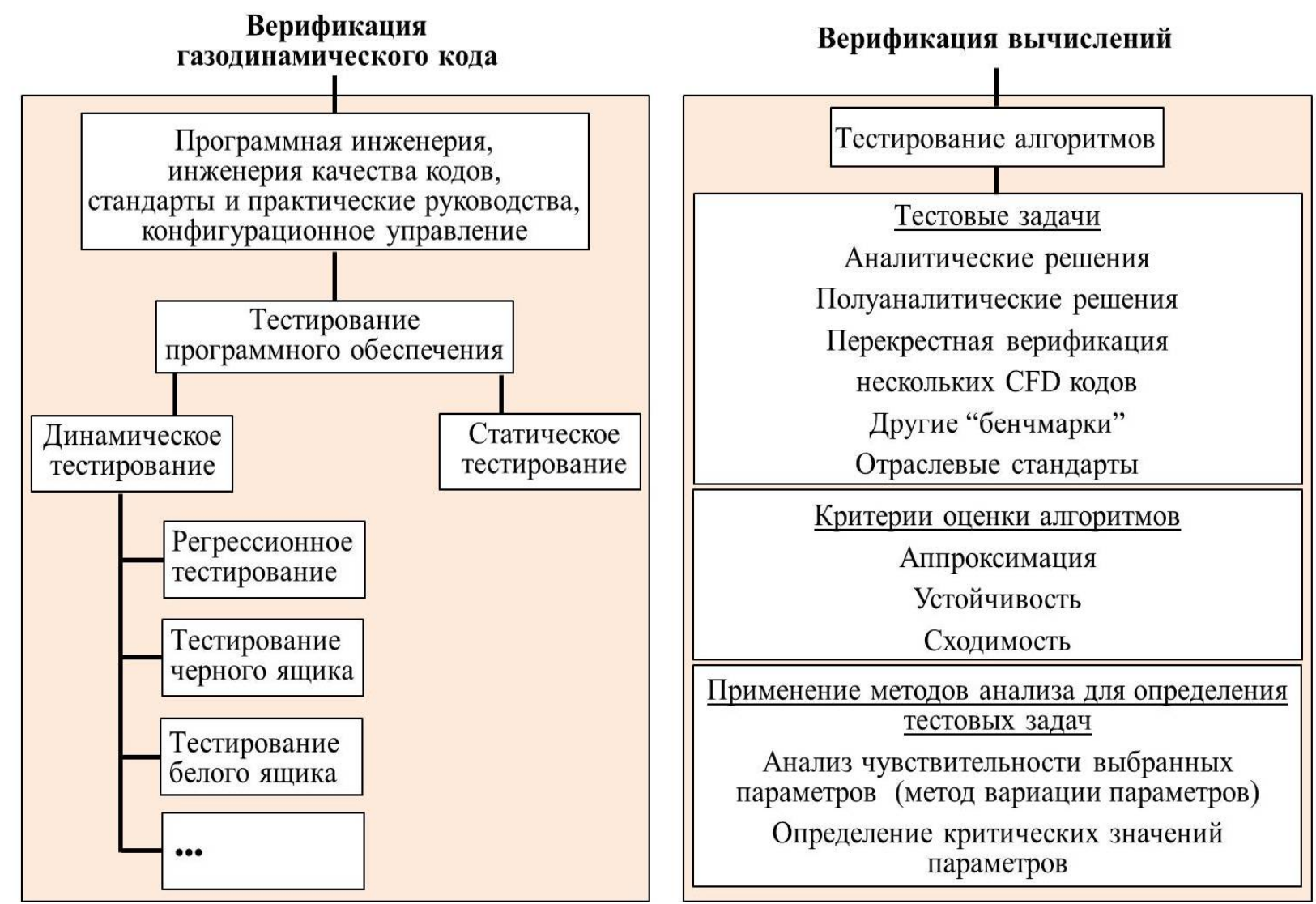

Рис. 3. Комплексный подход к верификации в вычислительной газовой динамике

Динамическое тестирование - тип тестирования, который предполагает запуск программного кода и анализ поведения приложения во время его работы. По результатам выполнения программы обнаруживаются ошибки в коде и потенциальные уязвимости, которые могут привести к ошибкам. Для выполнения динамического тестирования необходимо чтобы тестируемый программный код был написан, скомпилирован и запущен. В ходе динамического тестирования проверяются внешние параметры работы программы и ее производительность (загрузка процессора, использование памяти, время отклика и т.д.). Динамическое тестирование, в свою очередь, подразделяется на несколько видов, которые классифицируются по различным признакам [126,127]:

1) По доступу к исходному коду различают: тестирование методом белого ящика; тестирование методом черного ящика; тестирование методом серого ящика.

2) По объекту тестирования выделяют: функциональное тестирование; нагрузочное тестирование (тестирование производительности, тестирование стабильности); тестирование 
удобства использования; тестирование интерфейса пользователя; тестирование безопасности; тестирование совместимости.

3) По степени автоматизированности тестирование дифференцируют на ручное, автоматизированное и полуавтоматизированное.

4) По уровню тестирования проводят: компонентное (модульное) тестирование; интеграционное тестирование; системное тестирование.

5) По этапам жизненного цикла программного обеспечения выделяют: альфа тестирование, которое проводится до передачи пользователю (тестирование при приемке, тестирование новых функциональностей, регрессионное тестирование, тестирование при сдаче); бета тестирование, выполняющееся после передачи пользователю.

6) По критерию позитивности сценариев различают позитивное и негативное тестирование.

Из перечисленных типов наиболее широко в практике тестирования газодинамических кодов применяются: тестирование черного ящика, тестирование белого ящика, регрессионное тестирование (рис.3).

Техника тестирования черного ящика (функциональное тестирование), основана на работе исключительно с внешним интерфейсом тестируемого программного продукта и не предполагает знания его внутреннего устройства. Для газодинамических кодов данный тип тестирования обычно выполняется пользователями.

Тестирование белого ящика предполагает полную осведомленность обо всех особенностях тестируемого приложения, а также знание архитектуры программного обеспечения, поэтому доступно, как правило, только разработчику. В данном случае главной целью тестирования является выбор подходящих верификационных тестов на основе анализа внутренней структуры программы.

Регрессионное тестирование представляет собой повторное тестирование, выполняемое после внесения изменений в код программного продукта. Проводится для уверенности в том, что процесс модификации не внес или не активизировал ошибки в областях, не подвергавшихся изменениям.

Вторую ветвь структуры процесса верификации на рис.3 представляет тестирование алгоритмов. Данный процесс позволяет выявить логические ошибки построения алгоритмов и проверить корректность их работы на специально заданных тестовых примерах - задачах с известными входными данными и результатами. При этом основное внимание уделяется правильности численной реализации и верификации вычислений. Главной проблемой на данном этапе является выбор подходящих тестовых задач, которые используются для определения точности вычислений и оценки производительности по сравнению с тестовым случаем. Следует отметить, что в процессе тестирования не может быть доказано, что сложное научное программное обеспечение не содержит никаких ошибок и дефектов.

План тестирования алгоритмов строится на основе следующих принципов:

1) В ходе тестирования необходимо придерживаться четко поставленной цели и хорошо продуманной логике.

2) Методика и принципы, используемые при подготовке верификационных тестов, должны быть подробно описаны, а выбор тестовых задач - тщательно обоснован.

3) Требуется четко сформулировать критерии сравнительной оценки полученных результатов с эталонными, определить критерии “успеха" и “неудачи” при решении тестовой задачи.

Главной проблемой верификации газодинамических кодов остается разработка и подбор подходящих базовых тестов [19]. Как правило, в качестве последних используются тестовые задачи с известными точными аналитическими и полуаналитическими решениями. Аналитические решения системы дифференциальных уравнений, лежащей в основе концептуальной модели, обычно представлены виде бесконечных рядов, комплексных интегралов и асимптотических разложений. Для вычисления последних также применяются численные 
методы, однако точность этих расчетов может строго контролироваться. Недостаток использования высокоточных расчетных данных для оценки точности получаемых решений, в том, что прецизионные аналитические решения могут быть получены только для ограниченного круга задач в упрощенной постановке (тривиальной геометрии и простой физической модели).

В качестве эталонных также могут применяться частные решения дифференциальных уравнений, не имеющие явного практического применения, однако подходящие для сравнительной оценки точности кода. Тестовые задачи должны подбираться с учетом специфики инженерной или физической области, в которой программное приложение будет использоваться, и затрагивать наиболее важные проблемы. При отсутствии отраслевых стандартов набор верификационных тестов для программного обеспечения определяется разработчиками на основе его функциональных возможностей, с учетом требований и потребностей пользователей. Верификационные тесты могут быть также созданы искусственно, исходя из поставленных целей тестирования.

В вычислительной газовой динамике существует ряд общеизвестных “бенчмарков”. К таким тестам, например, относится классическая задача о сильном точечном взрыве, которая используется для проверки выполнения закона сохранения энергии в кодах, предназначенных для расчета ударно-волновых процессов [51]. Широко применяются тесты, направленные на проверку полного сохранения других физических величин, а также тесты на влияние граничных условий. Среди последних рассматривают тестовые варианты, проверяющие сохранение симметрии решения. Если в концептуальной модели предполагается плоскость симметрии, которая определяется путем задания нулевого нормального градиента для соответствующих переменных, то в процессе тестирования проводят вычисления для полной области, где условия симметрии не наложены. При этом должны быть получены идентичные результаты. Если математическая модель предполагает, что границы области решения концептуально удалены на бесконечность, то реальные размеры области численного интегрирования должны быть настолько большими, что ее дальнейшее увеличение никак не сказывается на решении. Вопросы, связанные с корректностью задания граничных условий, подробно рассмотрены в [51].

Для верификации часто применяют аналитические решения обыкновенных дифференциальных уравнений, которые являются частным случаем общей системы уравнений в частных производных при принятии ряда допущений и упрощений, позволяющих ввести автомодельные переменные. Примерами таких эталонных решений в газовой динамике являются: решение Блазиуса уравнений пограничного слоя при ламинарном обтекании плоской пластинки [148], решение Тэйлора - Маккола для невязкого течения около острых конусов [148] и т.д.

Верификацию также проводят с применением высокоточных решений системы дифференциальных уравнений в частных производных для определенных граничных условий. Например, используют решения, полученные для случаев: несжимаемого ламинарного течения над полубесконечной плоской пластиной $[70,74,78]$, несжимаемого ламинарного течения жидкости в квадратной каверне с движущейся крышкой [149-153], несжимаемого ламинарного течения около круглого цилиндра бесконечной длины $[44,45,57,154]$. Очевидно, что гарантируемая точность эталонных решений снижается при переходе от обыкновенных дифференциальных уравнений к уравнениям в частных производных.

В процессе количественного сравнения численного и высокоточного решений проводят оценку нормы ошибки (так называемой невязки). При этом для каждой зависимой переменной вычисляется точность ее определения (функционал невязки). Следует отметить, что требуемая точность численного решения может значительно варьироваться для различных переменных. 
На основании результатов моделирования простых тестовых случаев можно получить достоверную количественную оценку точности, с которой верифицируемый код решает поставленную задачу, однако подобные упрощенные постановки, как правило, далеки от практических приложений сложных вычислительных кодов.

В вычислительной газовой динамике, как и в любой области научных вычислений, “бенчмарки”, выступающие в роли эталонных оценочных критериев, должны быть стандартизованы. При разработке стандартов следует руководствоваться следующими принципами:

1) Ни одно численное решение не может считаться эталонным, пока аналогичный результат не будет получен другими независимыми исследователями, желательно с использованием различных численных подходов.

2) Эталонный тест должен иметь подробное описание, содержащее строгую формулировку задачи и исчерпывающие руководство по его применению. Описание должно быть задокументировано профессиональным сообществом, академическим институтом или некоммерческой организацией.

3) Четкая и однозначная постановка верификационной задачи должна быть стандартизована и обнародована.

4) Роль теста в комплексном плане верификации кода должна быть четко определена.

5) Необходимо сформулировать, обнародовать и стандартизировать требования к процедуре сравнения выходных данных верифицируемого кода с эталонным решением.

6) Необходимо определить, обнародовать и стандартизировать критерии "успеха" и “неудачи" при сопоставлении численного результата, полученного с использованием программного приложения, с точным решением.

В настоящее время специалистами в области вычислительной газовой динамики ведется активная работа по созданию базы верификационных и валидационных “бенчмарков" [19]. Усилия исследователей направлены на повышение доверия к промышленной CFD.

Как упоминалось выше, процесс верификации включает решение отдельных задач, относящихся к области программной инженерии и управления качеством программного обеспечения. В работе [144] предлагается рассматривать верификацию как управленческую деятельность, включающую соответствующие мероприятия (табл.1).

Имеется значительное количество специализированной литературы [54,144,155-159], посвященной различным аспектам программной инженерии и связанных с ней дисциплин: концепции инженерии качества; программным требованиям; проектированию, тестированию, сопровождению ПО; подходам к оценке качества ПО и способам его повышения; контролю и гарантии качества; стандартам в области инженерии качества и т.п.

Большая часть опубликованных работ в области инженерии качества программного обеспечения посвящена критическим компьютеризированным системам (системы управления безопасностью полетов, системы управления и защиты энергетических установок и т.д.). Последние управляются с помощью ПО, к которому должны предъявляться повышенные требования по надежности. В настоящее время наблюдается расширение области применения научных компьютерных вычислений в отраслях повышенного риска. Следовательно, сообщество разработчиков научного программного обеспечения может многое почерпнуть о стандартах программной инженерии из этих исследований.

\section{6. Методология валидации}

В процессе валидации определяют, насколько точно созданная компьютерная модель воспроизводит физическую действительность с позиции ее предполагаемого применения, посредством сравнения результатов вычислений с экспериментальными данными. При этом должно быть выполнено обязательное условие - вычислительная модель должна корректно воспроизводить математическую модель, отображающую отдельные стороны реального процесса. Поэтому, успех валидации зависит как от точности численного решения, так и от точ- 
ности эксперимента. Например, погрешность моделирования, возникшая вследствие недостаточной сходимости решения, может привести как к сокращению, так и к увеличению расхождения между расчетными и экспериментальными данными. Если такая вычислительная ошибка окажется достаточно серьезной, то она будет доминировать над этим несоответствием.

Таблийа 1

Задачи верификационной деятельности, направленные на достижение соответствия стандартам инженерии качества [144]

\begin{tabular}{|c|c|}
\hline $\begin{array}{c}\text { Виды верификационной } \\
\text { деятельности }\end{array}$ & Задачи \\
\hline $\begin{array}{l}\text { Управление верификацией } \\
\text { программного обеспечения }\end{array}$ & $\begin{array}{l}\text { Планирование верификации } \\
\text { Верификационный мониторинг (контроль заданных харак- } \\
\text { теристик поведения проверяемого ПО) } \\
\text { Анализ результатов } \\
\text { Составление отчета }\end{array}$ \\
\hline $\begin{array}{l}\text { Проверка соответствия тре- } \\
\text { бованиям к программному } \\
\text { обеспечению }\end{array}$ & $\begin{array}{l}\text { Изучение концептуальных положений и анализ норматив- } \\
\text { ной документации } \\
\text { Трассировка требований (установка связей и выявление за- } \\
\text { висимостей между требованиями) } \\
\text { Оценка требований к программному обеспечению } \\
\text { Анализ пользовательского интерфейса } \\
\text { Предварительное планирование системного тестирования } \\
\text { Подготовка отчета }\end{array}$ \\
\hline $\begin{array}{l}\text { Проверка соблюдения тех- } \\
\text { нического задания на разра- } \\
\text { ботку программного обес- } \\
\text { печения }\end{array}$ & $\begin{array}{l}\text { Трассировка требований } \\
\text { Оценка качества проектирования ПО } \\
\text { Анализ интерфейса } \\
\text { Предварительное планирование модульного тестирования } \\
\text { Предварительное планирование интеграционного тестиро- } \\
\text { вания } \\
\text { Составление отчетной документации }\end{array}$ \\
\hline $\begin{array}{l}\text { Проверка } \\
\text { кода }\end{array}$ & $\begin{array}{l}\text { Трассировка } \\
\text { Анализ исходного кода } \\
\text { Анализ интерфейса } \\
\text { Завершение подготовки к модульному тестированию } \\
\text { Составление отчета }\end{array}$ \\
\hline Модульное тестирование & $\begin{array}{l}\text { Выполнение модульного тестирования } \\
\text { Подготовка отчета }\end{array}$ \\
\hline $\begin{array}{l}\text { Интеграционное } \\
\text { тестирование программного } \\
\text { обеспечения }\end{array}$ & $\begin{array}{l}\text { Завершение подготовки к интеграционному тестированию } \\
\text { Выполнение интеграционного тестирования } \\
\text { Подготовка отчетной документации }\end{array}$ \\
\hline Системное тестирование & $\begin{array}{l}\text { Завершение подготовки к системному тестированию } \\
\text { Выполнение системного тестирования } \\
\text { Составление отчета }\end{array}$ \\
\hline $\begin{array}{l}\text { Тестирование установки } \\
\text { (проверка успешной ин- } \\
\text { сталляции и настройки) }\end{array}$ & $\begin{array}{l}\text { Аудит исполнения программного приложения } \\
\text { Подготовка отчета }\end{array}$ \\
\hline $\begin{array}{l}\text { Эксплуатация и сопровож- } \\
\text { дение программного обес- } \\
\text { печения }\end{array}$ & $\begin{array}{l}\text { Оценка влияния вносимых изменений } \\
\text { Повторное выполнение деятельности по управлению вери- } \\
\text { фикацией } \\
\text { Повторное выполнение всех этапов тестирования }\end{array}$ \\
\hline
\end{tabular}


Процедура валидации не решает вопрос о том, как модифицировать компьютерную модель, чтобы улучшить согласование между расчетными и экспериментальными результатами, однако требует выводов о точности модели для конкретных условий, реализующихся в рамках рассматриваемого валидационного эксперимента. В задачи валидации входит:

1) Идентификация и количественная оценка ошибки и неопределенности в концептуальной и компьютерной моделях;

2) Количественная оценка численной ошибки решения;

3) Оценка неопределенности в экспериментальном исследовании;

4) Сравнение экспериментальных и расчетных данных.

В процессе валидации точность измеряется по отношению к экспериментальным данным, которые принимаются за эталонные. Методология верификации не предполагает, что экспериментальные измерения более точные, чем результат вычислений. Постулируется только то, что эксперимент является наиболее объективным отражением реальности. Валидация требует, чтобы анализ ошибок и неопределенности проводился как с позиций математической физики, так и со стороны эксперимента. На рис. 4 показан процесс сопоставления данных численного моделирования с результатами поуровневого экспериментального исследования сложной инженерной системы.

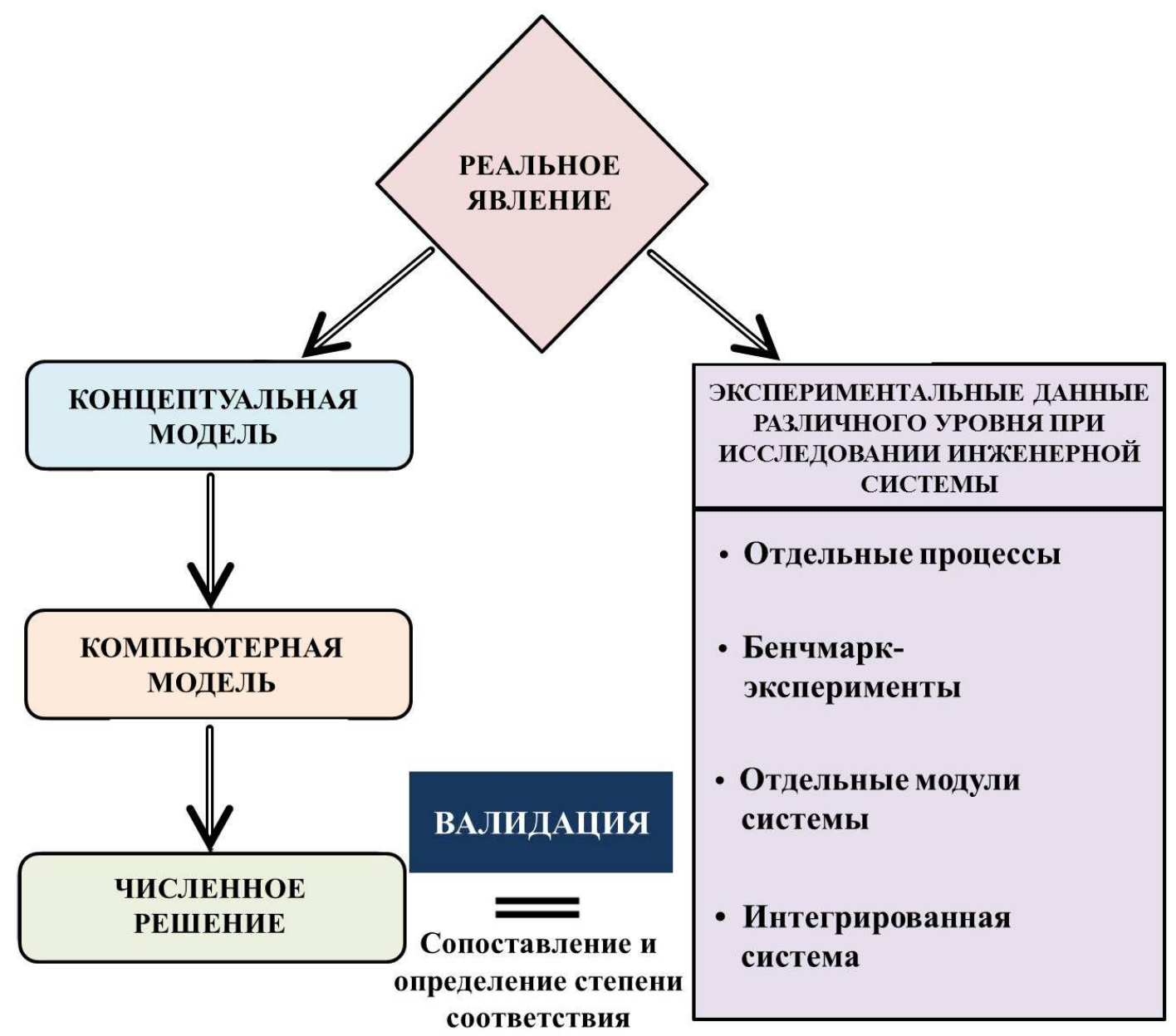

Рис. 4. Процесс валидации [122]

В процессе валидации приходится сталкиваться с рядом специфических проблем:

1) В реальной инженерной системе процессы протекают одновременно и взаимосвязано.

2) Измерения проводятся на инженерном оборудовании в реальных условиях и их точность ограничена. 
3) Обычно для реальной инженерной системы проблематично или невозможно определить количественно все условия эксперимента, необходимые для задания входных параметров валидируемой численной модели, например, термодинамические свойства многокомпонентной среды в сочетании с переменными, зависящими от времени граничными условиями и т.П.

4) Многие параметры, необходимые для численного сопровождения соответствующего эмпирического исследования не измеряются, даже анализ неопределенности задания условий эксперимента обычно не проводится.

Нецелесообразность постановки валидационных экспериментов для сложной инженерной системы, требует применения модульного принципа. Метод подразумевает разбиение комплексной системы на иерархическую структуру, содержащую три или более уровня (рис. 5): подсистемы, отдельные физические процессы или их группы, объединенные единой природой явлений. Искусство постановки подобных экспериментов заключается в необходимости выделения изучаемого процесса в “чистом виде". В этом случае преобразующее действие эксперимента (создание особенных условий специальными техническими средствами), направлено на разделение сложной совокупности взаимодействий на изолированные явления.

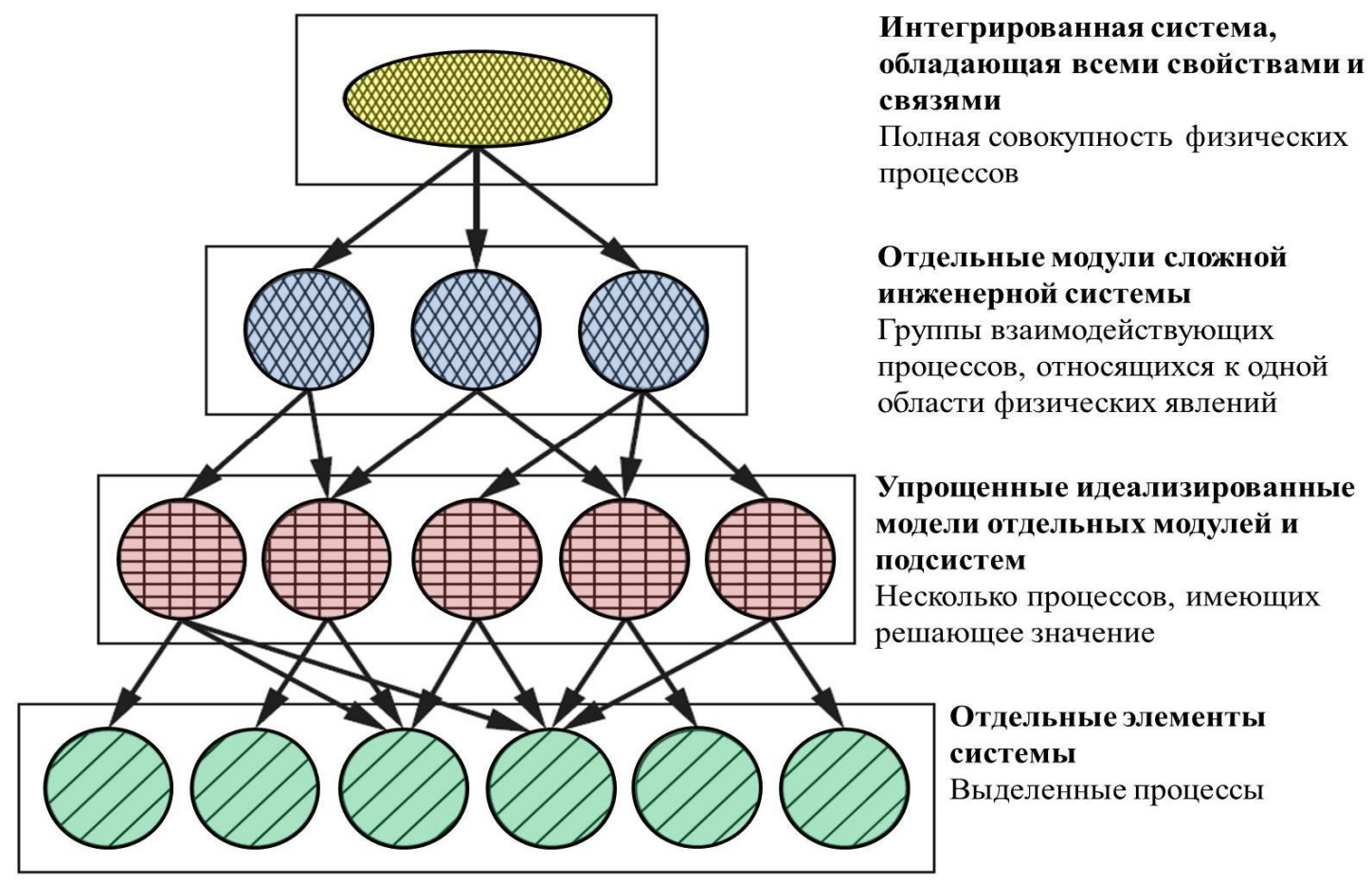

Рис. 5. Уровни валидационных данных [122]

В первом приближении сложную совокупность физических процессов, связанных с данным техническим объектом, разлагают на ряд независимых компонентов, каждый из которых содержит несколько взаимодействующих явлений, имеющих различную физическую природу (относящихся к газовой динамике, динамике конструкции, динамике твердых тел, физике химических реакций, акустике и т.д.). Для каждой отдельной группы перечисленных физических явлений имеется намного больше экспериментальных данных, в том числе с поправками на геометрические особенности.

Построение иерархической структуры процедуры валидации начинается с ее вершины. Верхний уровень занимает интегрированная инженерная система (см. рис. 5). Экспериментальные данные для сложной инженерной системы или ее отдельных модулей могут быть получены только в ходе реализации крупномасштабных программ. При этом усилия экспериментаторов направлены главным образом на решение задач функциональности, производительности, безопасности и надежности системы или ее подсистем. В условиях жесткой 
конкуренции между поставщиками аппаратного обеспечения, сжатых сроков и ограниченного бюджета невозможно получить полный набор параметров, необходимых для численного моделирования (начальных и граничных условий, а также беспристрастную и достоверную информацию о точности их определения).

Следующий ярус в рассматриваемой трехуровневой иерархии комплексной системы (см. рис. 5) занимают упрощенные идеализированные модели каждой подсистемы, которые имеют неполную функциональность. Для идеализированной подсистемы учитываются два или три взаимосвязанных физических процесса, например, турбулентность, горение, многофазность и т.д. Один или несколько второстепенных процессов, свойственных рассматриваемой подсистеме, могут быть исключены из рассмотрения. Идеализированные подсистемы имеют упрощенную геометрию. Сохраняются только те геометрические особенности, которые имеют решающее значение для исследуемой группы процессов. На данном уровне есть возможность оценки неопределенности в задании начальных и граничных условий, определения недостающих данных численного моделирования, характеристик материалов, проведения контроля условий окружающей среды, воссоздаваемых на экспериментальном оборудовании.

На нижнем уровне иерархической структуры валидационных данных расположены выделенные процессы. Только на данном уровне детализации можно обеспечить максимально высокую точность экспериментальных результатов. Изолированное изучение отдельного процесса требует специального тщательно протестированного аппаратного обеспечения. При этом используется самая простая геометрия. На данном этапе в ходе высокотехнологичных экспериментов подготавливают высокоточные валидационные данные и проводят анализ их неопределенности. Последние используются для критической оценки адекватности математических моделей. В ходе экспериментов аккуратно измеряются все входные данные, необходимые для последующего численного моделирования, точно определяются начальные и граничные условия.

Главная цель иерархической декомпозиции - представление исследуемого технического объекта в виде совокупности связанных физических моделей различной сложности и степени полноты описания процессов, соответствующих данной инженерной системе. Подобное рассмотрение позволяет спланировать серию экспериментов, результаты которых, будут использоваться для валидации специализированных компьютерных кодов, отвечающих за вычислительное сопровождение соответствующих структурных компонентов иерархии. При этом создаваемая древовидная структура должна ориентироваться на конкретное практическое приложение.

В настоящее время фокус разработки научного программного обеспечения смещается с универсальных вычислительных приложений, предназначенных для решения широкого круга задач, на коды, моделирующие отдельные группы физических процессов. Однако данный подход подразумевает, что при упрощении вычислительных моделей необходимо четко прослеживать связь между отдельными компонентами комплексной системы и учитывать реальные условия ее функционирования. При этом каждому элементу иерархии соответствует свой специализированный вычислительный код и серия валидирующих экспериментов. При переходе от верхних уровней структуры к нижним физическая сложность моделей уменьшается на один порядок. Под снижением порядка подразумевается понижение размерности или геометрической сложности, предположение стационарности, исключение из рассмотрения одного или нескольких физических процессов, пренебрежение взаимосвязанностью последних. Вычислительная модель всей системы строится на основе разноуровневых моделей ее составных частей в предположении о линейности связей между структурными элементами.

Главная проблема валидации состоит в грамотном и тщательном подборе экспериментов для каждого элемента иерархии, с целью получения качественных валидационных данных для проверки численных решений. Отбор осуществляется исходя из следующих принципов: 
1) Эксперименты должны быть физически выполнимыми с помощью доступного инструментального оснащения и финансового обеспечения в условиях жестких временных ограничений.

2) В ходе проведения эксперимента необходимо обеспечить возможность определения количественного отклика системы, в виде набора выходных параметров, фиксируемых датчиками, приборами, измерительными системами, для последующего использования при валидации вычислительных кодов.

3) Чем выше уровень рассматриваемой подсистемы в иерархической структуре инженерного объекта, тем сложнее постановка соответствующих валидирующих экспериментов. При переходе на нижние уровни акцент переносится с инженерно-технических аспектов на фундаментальные физические процессы, лежащие в основе функционирования системы.

Методику построения иерархической структуры комплексной инженерной системы удобно изложить на примере автономного аэрокосмического летательного аппарата. Наиболее общими элементами подобной системы являются: двигательная установка, планер, системы управления движением и навигацией (разбиение на подсистемы может быть и более подробным). Перечисленные модули располагаются на первом подуровне инженерной системы (см. рис. 5).

На следующем уровне “упрощенных моделей” осуществляется переход от конструктивных элементов системы к физическим процессам и явлениям, определяющим ее функционирование. Для рассматриваемого объекта этот ярус занимают отдельные группы связанных процессов, среди которых: абляция теплозащитного покрытия; прогрев конструкций летательного аппарата вследствие теплопроводности; ламинарное и турбулентное сверхзвуковое течение с учетом абляции; ламинарно-турбулентный переход и т.д. Экспериментальные исследования, соответствующие данному уровню декомпозиции исходной системы, посвящены изучению соответствующих комплексных процессов. На данном этапе постановка валидационных экспериментов проводится с полным или частичным учетом геометрических особенностей компонентов полной системы, поэтому получение высокоточных экспериментальных данных все еще остается серьезной проблемой.

Для валидационных задач самого нижнего яруса характерна максимально упрощенная геометрия. При этом из сложной физики течения выделяют одно или два явления. Например, исследуются: ламинарное или турбулентное сверхзвуковое течение около тел простой формы, ламинарное или турбулентное сверхзвуковое обтекание плоской пластины с вдувом, взаимодействие турбулентного пограничного слоя с ударной волной, ламинарно-турбулентный переход на телах простой геометрии, анизотропная теплопроводность и т.п. Данные, полученные из валидирующих экспериментов последнего яруса, имеют самую высокую достоверность и обладают наибольшей ценностью для разработчиков газодинамических кодов.

Валидационные эксперименты по исследованию идеализированных моделей и выделенных процессов (так называемые валидационные “бенчмарки”) планируются с учетом обеспечения требуемой точности измерения входных данных для последующего численного моделирования.

При отсутствии оборудования по измерению единичного параметра (например, какогото специфического свойства материала или среды), вычислитель задает разумные значения недостающих данных. Альтернативный подход, подразумевает задание распределений вероятностей неизмеренного параметра и его отклонений. На основе сделанных предположений выполняется серия вычислений при вариации неизвестного параметра. Затем полученное вероятностное распределение выходных данных сравнивается с результатами эксперимента.

На сегодняшний день в доступных описаниях “бенчмарк-экспериментов” отсутствует значительное число параметров. Дело в том, что раньше эксперименты проводились с целью лучшего понимания изучаемых физических явлений или определения интересующих параметров, но не для валидации вычислительных моделей. То есть результаты экспериментов 
использовались индуктивно для построения математических моделей физических явлений, а не для дедуктивной оценки их адекватности.

\section{7. Технология валидации газодинамических кодов}

Главным результатом процесса валидации является так называемая валидационная база данных, которая содержит воспроизводимые доказательства того, что компьютерная реализация обеспечивает определенную точность моделирования для рассматриваемого класса задач. Кроме этого, модель, прошедшая некоторый уровень валидации, должна обладать определенной прогностической силой. Последнее дает возможность использовать вычислительный код для прогнозирования состояния физической системы в условиях, для которых он не был валидирован. Взаимосвязь между валидацией и предсказательным моделированием поясняет рис. 6.

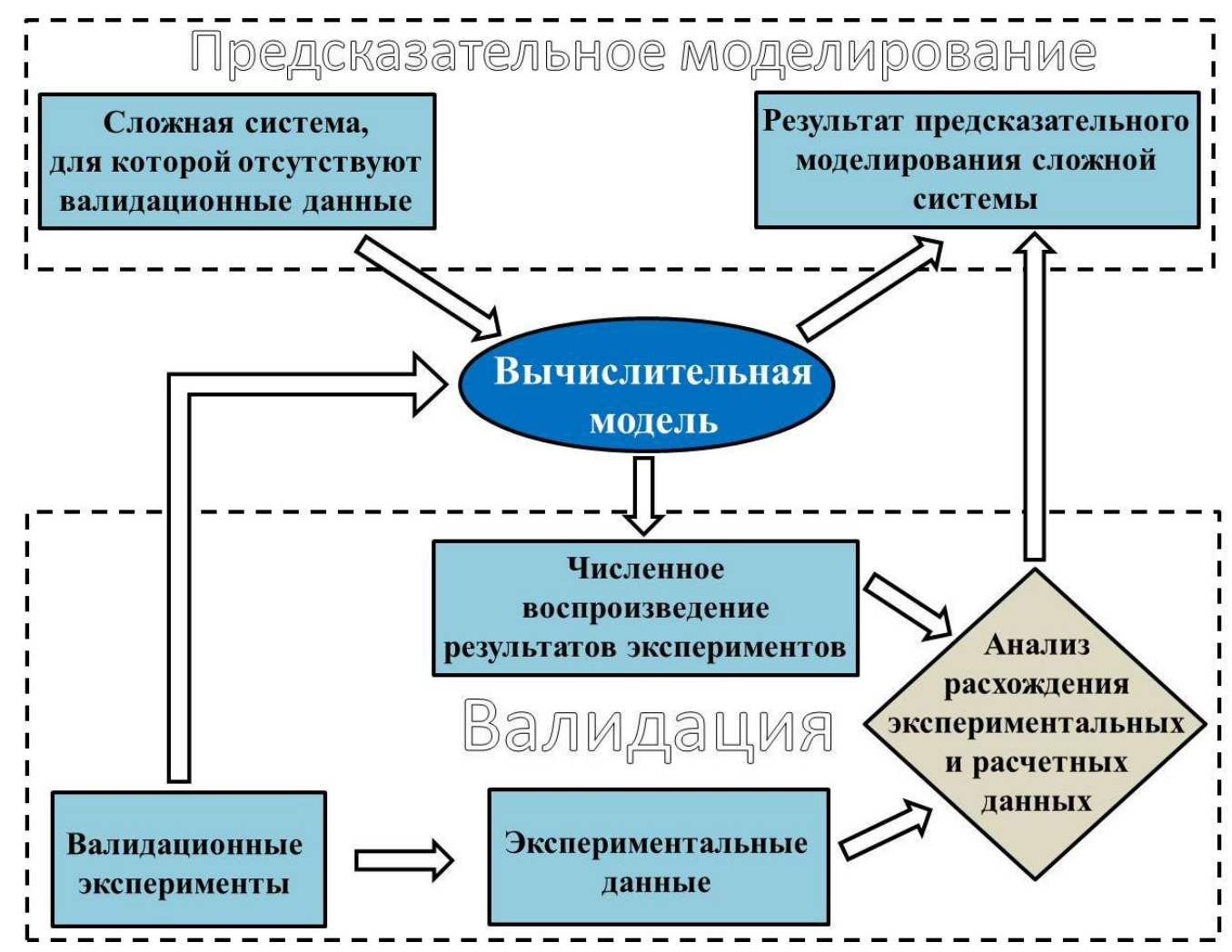

Рис. 6. Взаимосвязь между валидацией и предсказательным моделированием

Нижняя часть рисунка иллюстрирует процесс валидации. Блок “Валидационные эксперименты” содержит результаты ограниченной серии экспериментов, отражающих объективную реальность. Физические условия валидационных экспериментов используются в качестве входных параметры для численного моделирования, как начальные и граничные условия. Таким образом, компьютерная модель численно воспроизводит экспериментальные результаты. Полученные расчетные данные сопоставляются с результатами экспериментов, количественно оценивается их различие. Основываясь на величине этих расхождений и, исходя из понимания исследуемых физических процессов, делаются соответствующие выводы.

Верхняя часть рисунка посвящена прогностическому моделированию. Слева схематически изображена сложная система, адекватное моделирование которой является конечной целью всех усилий вычислителя. Для рассматриваемых комплексных систем экспериментальные реконструкции (валидационные данные) для интересующих условий, как правило, отсутствуют. В этом случае проводится предсказательное моделирование комплексной системы с использованием вычислительной модели при заданных условиях моделирования. 
Вопрос доверия к расчетным данным решается на основе расхождения численных и эмпирических результатов, полученных для валидационных задач, с учетом понимания сущности изучаемых явлений.

Таким образом, численная модель и ее валидационная база данных (валидированная численная модель) подобна классической научной теории. Однако, прогностическая сила численных моделей существенно слабее. Принципы компьютерного моделирования строятся на основе тех же логических законов, что и традиционные физические теории. В отличие от физика-теоретика, физику-вычислителю приходится сталкиваться с дополнительными проблемами разработки алгоритмов дискретизации и качества расчетных сеток, а также решать практические вопросы, связанные с компьютерным оборудованием, программным обеспечением, операционной системой, надежностью исходного кода. Кроме этого вычислитель должен обладать навыками аналитика.

Адекватность численной модели напрямую зависит от степени понимания моделируемого физического процесса и его сложности. Чем глубже понимание физических и математических аспектов моделируемого явления, тем надежнее численное решение.

В качестве примера можно привести ламинарное, несжимаемое, однофазное, одножидкостное, нетеплопроводное, нереагирующее, ограниченное стенкой ньютоновское течение. Для неподвижной геометрии (фиксированных границ) в математической постановке рассматриваемой задачи остается только один безразмерный параметр - число Рейнольдса. Однако даже для такого простого потока (поскольку число Рейнольдса и геометрия могут изменяться), характер течения может существенно трансформироваться - стать трехмерным и нестационарным. Суть в том, что по мере усложнения процесса предсказательная сила валидированной с использованием доступной базы данных численной модели ослабевает, причем, практически непредсказуемым образом.

В ходе планирования валидационной деятельности необходимо решить следующие задачи:

1) Задать количественную меру сравнения численных результатов и экспериментальных данных.

2) Выбрать или провести наиболее подходящие валидационные эксперименты.

3) Определить параметры валидационного эксперимента.

Для определения количественной меры несоответствия численных и эмпирических данных используются понятия "валидационная ошибка" и “неопределенность". Пусть $u$ произвольная величина отклика системы, которая вычисляется и измеряется экспериментально, а $\Delta$ - разность между объективным (истинным) значением $u_{\text {nature }}$ и численным результатом $u_{\text {discrete }}[122]$ :

$$
\Delta=\left(u_{\text {nature }}-u_{\text {discrete }}\right)
$$

Эта разность может быть представлена следующим образом [122]:

$$
\Delta=\left(u_{\text {nature }}-u_{\text {exp }}\right)+\left(u_{\text {exp }}-u_{\text {exact }}\right)+\left(u_{\text {exact }}-u_{\text {discrete }}\right)
$$

Здесь $u_{\text {eхр }}$ - величина, измеренная в эксперименте; $u_{\text {eхасt }}$ - точное решение системы уравнений математической модели для заданного набора начальных и граничных условий; $u_{\text {discrete }}-$ численное решение дискретной аппроксимации данных уравнений при тех же условиях, полученное с использованием конкретного кода на определенной вычислительной машине. Последняя разность в (13) представляет собой погрешность численного решения (2), которая была определена ранее. С учетом (2) соотношение (13) может быть представлено в виде

$$
\begin{gathered}
\Delta=E_{1}+E_{2}+E_{3}+E_{4}, \\
E_{1}=\left(u_{\text {nature }}-u_{\exp }\right), \quad E_{2}=\left(u_{\exp }-u_{\text {exact }}\right),
\end{gathered}
$$




$$
E_{3}=\left(u_{\text {exact }}-u_{h, \tau \rightarrow 0}\right), \quad E_{4}=\left(u_{h, \tau \rightarrow 0}-u_{h, \tau, I, c}\right)
$$

Слагаемое $E_{1}$ представляет собой ошибку, вносимую в процессе экспериментального измерения физического параметра. В соотношении (14) под истинным значением $u_{\text {nature }}$ понимается детерминированная величина, которая полностью определяется и неизменно реализуется при заданном фиксированном наборе физических условий (явления бифуркации и хаотичности не рассматриваются). На самом деле $u_{\text {nature }}$ будет принимать случайные значения, так как один и тот же комплекс физических условий не может воссоздаться повторно. Например, при выполнении продувок в аэродинамических трубах, входные и выходные параметры потока, как правило, поддаются достаточно точному контролю, однако они не являются детерминированными. В летных экспериментах атмосферные условия являются полностью неконтролируемыми, и в данном случае возникает необходимость в максимально точной оценке параметров состояния атмосферы, которые варьируются по времени и пространству. Величина $u_{\text {exр }}$ является явно случайной, вследствие неопределенности, которая неизбежно возникает в ходе инструментального измерения.

Таким образом, значение $E_{1}$ рассматривается как случайное, однако оно может быть отличным от нуля из-за смещения среднего значения вследствие случайных или систематических ошибок. Если первые остаются постоянными или закономерно изменяются при повторных измерениях одной и той же величины, то вторые меняются случайным образом.

Источниками систематических ошибок в газодинамических измерениях могут быть: температурный дрейф датчика давления, погрешность усилителя и др.

Слагаемое $E_{2}$ учитывает все ошибки и неопределенности, возникающие при попытке описать экспериментально воспроизводимое явление с помощью системы непрерывных дифференциальных уравнений в частных производных. Последняя включает набор граничных и начальных условий, а также другие параметры, необходимые для моделирования соответствующего эксперимента. Данную составляющую обычно называют ошибкой моделирования. Большинство таких ошибок - это вводимые аппроксимации и принимаемые допущения (например, приближения сплошной среды, несжимаемости низкоскоростного течения, предположение о постоянстве числа Прандтля в турбулентном потоке, приближения различных моделей турбулентности и т.п.). В рассматриваемое слагаемое так же входят: неучтенные погрешности; неопределенность задания скоростей химических реакций, параметров турбулентности, шероховатости поверхности и т.п.; ошибки задания входных параметров, определяющих термодинамические свойства среды; неопределенность задания необходимых для расчета исходных величин, неизмеренных в эксперименте.

\section{8. Планирование валидационных экспериментов}

Задачи классических опытных исследований существенно отличаются от задач валидационных экспериментов, что должно учитываться при планировании и проведении последних. Традиционные эксперименты, в свою очередь, можно разделить на три группы. К первой категории относятся исследования, направленные на проверку понимания фундаментальных физических процессов, например, измерение характеристик турбулентности, детальное изучение кинетики и механизмов химических реакций в процессах горения, исследование течений в условиях термохимической неравновесности и т.д. Главная цель второго типа классических экспериментов - создание новых или развитие существующих математических моделей для достаточно изученных явлений. В качестве примеров можно привести следующие эксперименты: измерение параметров, характеризующих интенсивность протекания химических реакций в реагирующем потоке; определение пульсационных параметров потока для последующего моделирования турбулентности; определение излучательной способности частиц примеси или поверхностей. К третьему классу относятся испытания и тестирование, которые посвящены обеспечению и повышению надежности, безопасности, эффективности 
сложных технических объектов, их подсистем и отдельных компонентов (испытания газотурбинного или ракетного двигателя, новой конструкции камеры сгорания). К последней группе также следует отнести продувки в аэродинамических трубах с целью определения аэродинамических сил и моментов, действующих на поверхность летательного аппарата и создаваемых его управляющими поверхностями.

В отличие от рассмотренных выше традиционных эмпирических исследований в процессе валидационного эксперимента получают данные, с помощью которых устанавливается адекватность, определяется предсказательная точность, оцениваются возможности вычислительной модели. Таким образом, деятельность по планированию, проведению и анализу результатов валидационного эксперимента осуществляется с целью определения количественных показателей способности математической модели и ее программной реализации моделировать хорошо изученный физический процесс.

До недавнего времени вычислительные методы рассматривались как вспомогательный инструмент, обеспечивающий математическое сопровождение экспериментальных исследований. В последнее десятилетие в области новых технологий наблюдаются тенденции к использованию инженерных систем, полностью спроектированных с применением численного моделирования, при этом эксперимент все чаще стоит на службе вычислительной газовой динамики. Следовательно, разработка единой концепции проведения валидационных экспериментов является первостепенной задачей сообщества исследователей в данной области.

Руководящие принципы планирования и проведения валидационной деятельности сформулированы в работах $[42,93,111-113]$. Несмотря на то, что предложенные методические рекомендации создавались в рамках совместных расчетно-экспериментальных исследований в аэродинамической трубе, они справедливы для широкого класса валидационных экспериментов [89-92, 111-113, 160]. Разработанную концепцию можно кратко изложить в виде ряда положений.

Планирование валидационного эксперимента должно проводиться в тесном сотрудничестве между экспериментаторами, создателями математической модели, разработчиками и пользователями компьютерного кода. При этом все участники кооперации должны быть полностью осведомлены о недостатках, слабых и сильных сторонах каждого подхода. На первый взгляд данное требование может показаться легко выполнимым, однако его крайне сложно осуществить на практике. Если исследовательские команды вычислителей и экспериментаторов принадлежат разным организациям и их совместная деятельность скреплена контрактом, то в условиях жесткой конкуренции и борьбы за финансирование, владельцы испытательных стендов будут очень неохотно раскрывать информацию о недостатках и дефектах оборудования.

В ходе валидирующего эксперимента необходимо отслеживать и регистрировать все ключевые процессы и входные данные, которые задают набор начальных и граничных условий, требуемых для полного определения задачи численного моделирования. При этом учитываются: пространственная размерность, геометрическая сложность и характер временной зависимости параметров верифицируемой модели. Следует отметить, что в реальном физическом эксперименте приходится иметь дело с трехмерными объектами и нестационарными процессами, а стационарность, двухмерность, осесимметричность или сферическая симметрия - всего лишь упрощающие теоретические допущения. Поэтому, задача экспериментатора - максимально точно воспроизвести поведение именно данной модели, реализованной в программном коде. Инструментальное оснащение эксперимента должно быть достаточным для получения необходимого набора данных и обеспечения достаточной точности и пространственного разрешения. Вычислитель, в свою очередь, должен учитывать ограниченность возможностей эксперимента и оборудования, создавая максимально реалистичные и полные математические модели, определять физически реализуемые граничные условия. Глубокое по- 
нимание и преодоление трудностей, с которыми приходится сталкиваться экспериментаторам и вычислителям, возможно только при совместном планировании валидационных экспериментов.

Главной причиной, по которой ранее опубликованные экспериментальные работы имеют ограниченное применение для валидации газодинамических кодов, является недостаточность информации, необходимой для подготовки исходных данных численного моделирования, задания начальных и граничных условий. Подробные университетские или корпоративные отчеты, которые не ограничены форматами научной статьи или тезисов, как правило, в открытом доступе не выкладываются.

Понятие о требуемом уровне детализации можно получить из приведенного ниже списка необходимых количественно фиксируемых условий валидационного эксперимента при выполнении продувки летательного аппарата в аэродинамической трубе и соответствующего расчета. Таким образом, неполный перечень измеряемых параметров будет выглядеть следующим образом:

1) Точно измеренные фактические габариты модели, а не номинальные размеры объекта с соответствующими отклонениями от круглости, прямолинейности и т.п., указанные в чертежах (к последним вычислители не всегда имеют доступ).

2) Шероховатость поверхности, включая мелкие несоответствия и незначительные дефекты, всегда присутствующие на реальном изделии.

3) Положение точки ламинарно-турбулентного перехода во всем диапазоне моделируемых условий полета (параметров набегающего потока, углов атаки, отклонениях управляющих поверхностей).

4) Параметры турбулентности набегающего потока.

5) Фактические места размещения всех измерительных приборов и датчиков, а не их положения, указанные в рабочих чертежах.

6) Точка измерения параметров невозмущенного потока в аэродинамической трубе (при дозвуковых скоростях обтекания).

7) Давление на стенке рабочей камеры аэродинамической трубы в месте, соответствующем границе области численного решения рассматриваемой задачи.

8) Точные размеры стоек, державок и других крепежных элементов, необходимых для установки и перемещения модели летательного аппарата в рабочей части аэродинамической трубы.

Задачу усложняют деформации, которым подвергаются корпус, крылья и другие элементы конструкции аппарата под воздействием аэродинамических нагрузок, в том числе аэродинамического нагрева (для сверхзвуковых скоростей).

В процессе продувок важно обеспечить точный и надежный контроль параметров набегающего потока. Обычно это следующие величины, усредненные по объему рабочей камеры: число Маха, полное и статическое давление, полная и статическая температура. Для турбулентных течений должны быть измерены такие характеристики, как масштаб вихревых структур, интенсивность и частота турбулентных пульсаций. Уровень турбулентности определяет качество потока, формируемого аэродинамической установкой, поэтому владельцы экспериментальных стендов неохотно делятся соответствующей информацией. Однако для сравнения экспериментальных данных с расчетными результатами, полученными с использованием сложных моделей турбулентности и моделей ламинарно-турбулентного перехода, эти параметры имеют решающее значение. Для сверхзвуковых аэродинамических труб экспериментально измеренная неоднородность входного потока может учитываться при моделировании в виде пространственно-зависимых граничных условий перед фронтом головной ударной волны. Хотя для экспериментальных установок, формирующих качественное течение, такая предосторожность является излишней, учет неоднородности просто необходим в случае высокоэнтальпийных аэродинамических труб. 
Для дозвуковых аэродинамических установок постановка граничных условий представляет еще более сложную проблему. Вычислителю нужно решить - моделировать течение во всей рабочей камере или принять ее размеры бесконечно большими. Для этого необходимо оценить различие в измеренных экспериментально и полученных путем численного моделирования значениях соответствующих параметров в обоих случаях.

В ходе проведения валидационного эксперимента желательно достичь наиболее полной интеграции экспериментального и вычислительного методов. Тесная интеграция обеспечивает взаимную компенсацию недостатков и повышение эффективности каждого подхода и приводит к качественно новым результатам. Данные, полученные с использованием одного подхода (будь то натурный или вычислительный эксперимент), позволяют повысить точность и возможности другой методики, а также добиться лучшего понимания исследуемых процессов.

В качестве примера такой синергии можно рассмотреть расчетно-экспериментальные исследования в сверхзвуковой аэродинамической трубе, способной воссоздать условия ламинарного обтекания объектов, как простой, так и сложной конфигурации потоком совершенного газа. Привлечение газодинамических кодов позволяет получить высокоточные решения для случая простой геометрии при малых углах атаки. Численные результаты используются для калибровки входного потока, а также обнаружения и устранения дефектов аэродинамической установки, неисправностей измерительных приборов и систем регистрации данных. При переходе к продувке реальной конфигурации летательного аппарата результаты экспериментального исследования сложного трехмерного течения будут обладать большей степенью достоверности, чем расчетные данные и могут использоваться для тестирования вычислительных моделей и расчетных кодов. Возможности вычислительного подхода могут быть использованы при планировании эксперимента с целью совершенствования конструкции установки. Кроме этого, предсказание положения зон максимальных тепловых нагрузок и областей взаимодействия падающих ударных волн с пограничным слоем помогает оптимально размесить контрольно-измерительную аппаратуру.

Тесное взаимодействие при подготовке валидационного эксперимента не должно отражаться на независимости получения экспериментальных и расчетных результатов. На этапе валидации стадия калибровки параметров вычислительной модели предполагается пройденной, поэтому подгонка значений под экспериментальные данные является недопустимой. Команде вычислителей предоставляется полная информация о параметрах численного моделирования, начальных и граничных условиях. Обмен результатами исследований, сравнительная оценка и анализ проводятся на заключительной фазе валидации с участием обеих исследовательских групп.

Обычно в результате сопоставления отмечается удовлетворительное согласие опытных и расчетных данных по нескольким позициям, по остальным параметрам выявляются расхождения. После выяснения и устранения причин несоответствия проводится еще одна серия совместных расчетно-экспериментальных исследований. Такой итеративный процесс чрезвычайно полезен как вычислителям, так и экспериментаторам для более глубокого понимания опытных и расчетных результатов.

Валидационный эксперимент должен предусматривать иерархическую организацию экспериментальных измерений, что подразумевает определение как интегральных, так и локальных (распределенных) параметров. Очевидно, что при переходе от интегральных величин к местным сложность поставленной перед вычислителями и экспериментаторами задачи значительно возрастает. При валидирующих экспериментах на аэродинамических трубах обычно используется следующая иерархия:

1) Нахождение интегральных аэродинамических сил и моментов, действующих на летательный аппарат.

2) Определение сил и моментов, создаваемых посредством отклонения управляющих поверхностей на различные углы. 
3) Получение распределений давления по поверхности.

4) Измерение тепловых потоков и касательных напряжений.

5) Определение давления, температуры и компонент скорости в возмущенном поле течения вблизи обтекаемого тела.

6) Получение распределений рейнольдсовых напряжений в возмущенном потоке.

Каждый следующий уровень предложенной структуры дает более детальное понимание исследуемого процесса и вытекает из предыдущего, являясь его составной частью, либо временной или пространственной производной. Данная классификация может использоваться для оценки достоверности валидируемой модели. При этом достигнутая степень адекватности определяется соответствующим набором параметров, по которым получено неплохое согласие. Например, при удовлетворительном соответствии расчетных и экспериментальных данных по нормальной силе, полученные значения тепловых потоков могут существенно различаться. Хорошо известно, что для большинства конфигураций нормальные силы удается вычислить достаточно точно, даже в рамках модели невязкого течения, в то время как для расчета величин тепловых потоков необходима более высокая физическая точность (особенно для турбулентных течений). Для достижения одинаковой степени точности при определении этих двух величин нужно использовать сетки различной подробности. Для относительно простого случая сжимаемого, ламинарного, безотрывного обтекания треугольного крыла под малым углом атаки, расчет тепловых потоков потребует 100-1000-кратного увеличения числа узлов [160]. Неопределенность измерения величин, классифицированных в соответствии с изложенным выше принципом, возрастает приблизительно в два раза [160] при переходе к следующему уровню иерархии.

Разработка относительно новых экспериментальных методик, таких как PIV-метод $[161,162]$ и PLIF-технология [163] позволили существенно расширить возможности измерения распределенных величин в потоке и увеличить потенциал валидирующих экспериментов. Лазерная анемометрия по изображениям частиц или так называемый PIV (Particle Image Velocimetry)-технология обеспечила визуализацию двумерных векторных полей скорости потоков жидкости или газа путем цифровой обработки изображений введенных в поток частиц. Метод PLIF (Planar Laser Induced Fluorescence) позволил регистрировать мгновенное распределение температуры рабочей среды в плоском сечении потока по интенсивности свечения растворенного в ней флуоресцентного красителя, освещаемого лазерным ножом.

Следует еще раз подчеркнуть, что валидационный эксперимент должен включать измерения величин, относящихся к нескольким уровням приведенной выше иерархии, что особенно критично при исследовании сложных комплексных процессов.

В ходе валидационного эксперимента должны проводиться оценка и анализ случайных и систематических ошибок. Консультативной группой НАТО по аэрокосмическим исследованиям и разработкам AGARD (Advisory Group for Aerospace Research and Development) coздана стандартная методика оценки погрешности измерения в аэродинамических трубах [82], опубликованная в официальном документе [164] и работе [165]. Методология предполагает поэтапное отслеживание и определение случайной и систематической составляющих ошибки измерений на каждой стадии преобразования потока данных - от входного сигнала, подаваемого на датчик, до конечного результата измерений.

Альтернативный подход к определению экспериментальной погрешности разработан группой исследователей Сандийских национальных лабораторий США [89,90,112,160]. Предложенная технология подразумевает проведение многократных испытаний и большого числа измерений интересующей физической величины. На основании полученных результатов вычисляются как случайные, так и систематические ошибки. Анализ данных проводится с использованием классических статистических методов [166]. Таким образом, традиционный подход $[164,165]$ можно рассматривать как априорный, в то время как альтернативная технология $[89,90,112,160]$ ориентирована на апостериорную оценку погрешности. 
Интуитивно понятно, что множественные измерения, выполненные в идентичных условиях, позволяют уменьшить влияние случайных погрешностей на результат измерений, так как приблизительно равные по модулю положительные и отрицательные отклонения компенсируют друг друга. Кроме того, становится возможным оценить систематическую составляющую погрешности, которая, как правило, превышает случайную ошибку.

Альтернативная методика также предполагает дублирование валидационного эксперимента на различном оборудовании. При этом получение удовлетворительного согласия между результатами продувок одной и той же модели при аналогичных параметрах набегающего потока в двух различных аэродинамических установках может гарантировать отсутствие систематических ошибок, вызванных неучтенными факторами. В ходе испытаний в аэродинамических трубах источниками систематических ошибок обычно являются: конденсация, скос потока и т.п. Использование различных установок также помогает выявить и устранить неточности калибровки входного потока.

В рамках экспериментов $[89,90,112,160]$ были проведены исследования поверхностного распределения давления и интегральных аэродинамических характеристик обтекаемой модели на двух аэродинамических установках научно-исследовательского инженерного центра ВВС США им. Арнольда и аэродинамической трубе Сандийских национальных лабораторий. Результаты продувок показали, что даже для этих аэродинамических установок, которые характеризуются высоким качеством потока, самая большая составляющая общей погрешности была обусловлена неравномерностью скорости рабочей среды. Значение ошибки, вносимой неоднородностью течения в рабочей части, могло в три раза превышать величину погрешности средств измерений. К последним были отнесены ошибки, вызванные: нелинейностью и гистерезисом тензорезисторного датчика силы; температурным смещением чувствительности, температурным смещением нуля; погрешностью преобразования данных; неточностью ориентации модели по углу тангажа, крена, рыскания; вариацией параметров входного потока в рабочей секции от запуска к запуску; погрешностью преобразователей давления.

\section{9. Количественная оценка неопределенности вычислений в процессе валидации}

Как упоминалось выше, валидационный процесс включает обязательное определение погрешностей и неопределенностей как экспериментального, так и численного подходов. При моделировании валидационного эксперимента часто возникают ситуации, когда какойнибудь параметр в исходной системе уравнений или граничных условиях не может быть определен экспериментально с достаточной точностью. Например, при выполнении серии летных экспериментов приходится сталкиваться с неконтролируемыми параметрами окружающей атмосферы. В этом случае имеет место стохастическая параметрическая неопределенность исходных данных численного моделирования, которая требует проведения, так называемых недетерминированных расчетов.

Один из стандартных подходов предполагает выбор разумного значения недостающего параметра на основании тех или иных предположений. Однако этот метод применим, если диапазон изменения параметра невелик, а результаты не очень чувствительны к его значениям. Если несколько величин не поддаются опытному определению, то синтез соответствующих погрешностей может значительно повлиять на конечный результат.

Простейший метод учета неопределенности задания входного параметра в процессе моделирования включает три этапа [167]. На первом шаге неопределенность интересующей величины представляется в виде некоторой заданной функции распределения вероятностей. Распределение может быть вычислено непосредственно при наличии достаточного объема экспериментальных данных.

На втором этапе с использованием методов Монте-Карло формируют ансамбль входных данных - выборку случайных значений рассматриваемого недетерминированного пара- 
метра, который подчиняется заданному закону распределения [167,168]. Полученные значения используются для проведения серии расчетов. Реализация метода подразумевает выполнение так называемых ансамблевых вычислений, вместо единичного или точечного моделирования, что требует больших вычислительных затрат. Ввиду сложности современных инженерных задач развитие данного подхода сталкиваются со значительными трудностями.

На заключительной, третьей, стадии проводится количественная оценка неопределенности выходных параметров. Данный этап включает анализ результатов расчетной серии методом статистического вывода, который позволяет получить распределение вероятностей значений интересующей выходной переменной на основе стохастических распределений одного или нескольких входных параметров. Такие статистические оценки используются для сравнения результатов вычислений с экспериментальными измерениями. Одной из наиболее значимых характеристик найденного распределения вероятностей является математическое ожидание, которое вычисляется как среднее значение данной величины. Последнее удобно использовать для сопоставления с усредненным результатом нескольких измерений. Не менее важно определить дисперсию распределения выходного параметра, которая характеризует разброс его значений относительно математического ожидания и может интерпретироваться как мера неопределенности отклика системы (мера рассеяния вычислительного результата) на заданную неопределенность входных данных.

При этом ошибочно считать, что математическое ожидание рассматриваемого выходного параметра можно получить на основании одного набора средних значений случайных входных величин, заданных соответствующими распределениями вероятностей. Искомая характеристика может быть найдена лишь посредством выполнения целого ансамбля вычислений с использованием специальных рациональных методик статистической оценки средних значений [169]. Следует также учитывать неточность определения среднего, вносимую допущением о том, что принятая вычислительная модель является адекватной, а используемые вероятностные распределения входных параметров достаточно точно описывают поведение последних.

Попытки количественной оценки вычислительной неопределенности на основе анализа чувствительности вычислительной модели и ее реализации были предприняты в работах [170-173]. Анализ чувствительности проводился на основании серии расчетов и состоял в определении влияния изменения одного из входных параметров модели на конечный результат (выбранные выходные переменные). В ходе оценки чувствительности решения на возмущения исходных данных вычислялся диапазон вариации выходных величин при конкретном изменении выбранной входной переменной и постоянстве остальных входных параметров. Кроме этого исследовалась чувствительность заданного выходного параметра к возмущениям различных исходных величин.

На сегодняшний день изложенная методика недетерминированных вычислений широко применяется в климатологии, при моделировании распространения токсичных отходов и загрязняющих веществ [174-177], однако ее использование для решения современных задач вычислительной газовой динамики ограничено высокой вычислительной сложностью последних.

\section{0. Метод проверки статистических гипотез}

На заключительном этапе процесса валидации необходимо проанализировать полученное расхождение экспериментальных и расчетных данных с учетом выполненных оценок ошибок и неопределенностей. На основе результатов анализа дается заключение об успешности проверки достоверности вычислительной модели.

В статистическом анализе принятие подобных решений о том, противоречит ли теоретическое утверждение или модель наблюдаемой выборке экспериментальных данных осуществляется на основе хорошо развитой и общепринятой методики, называемой проверкой статистических гипотез [178]. Обычно данный подход применяется при выборе между двумя 
конкурирующими моделями для минимизации риска получения ошибочного решения. Для проверки гипотезы используют статистические критерии, позволяющие принять или опровергнуть гипотезу. Простой пример - это проверка статистической гипотезы о симметрии монеты путем ее подбрасывания. Гипотеза о том, что монета "идеальная" предполагает равновероятное выпадение "орла" и "решки”. Конкурирующая гипотеза основывается на том, что монета “неправильная". Анализ результата эксперимента по бросанию монеты (процентное соотношение “орлов” и “решек”) с определенным числом попыток $N$ позволяет оценить уровень доверия (доверительную вероятность) одной или другой гипотезы. При этом с ростом $N$ достоверность вывода возрастает.

При проверке статистических гипотез существует риск прийти к неверным выводам. В процессе проверки неподтвержденных гипотез могут быть допущены ошибки двух типов. К ошибкам первого типа относятся риски получения ложных выводов о неадекватности вычислительной модели в процессе валидации. Проблема может быть обусловлена как теоретическими, так и экспериментальными аспектами. Например, если неверный численный результат был получен из-за недостаточной сеточной сходимости, то неудовлетворительный итог сравнения с экспериментом приведет к неверным выводам относительно достоверности модели. Неудачный результат сопоставления опытных и расчетных данных может быть получен также вследствие неучтенной систематической погрешности эксперимента.

Ошибка второго рода приводит к принятию модели, которая на самом деле является неадекватной. Причина ошибок второго типа также может крыться как в расчете, так и в эксперименте. В этом случае хорошее согласие расчетных и опытных данных может быть получено вследствие допущения взаимно компенсирующих ошибок в вычислениях, что часто встречается на практике при моделировании сложных процессов. Похожая ситуация может наблюдаться и в экспериментальных исследованиях, когда неучтенная систематическая ошибка нивелирует недопустимое расхождение данных.

Ошибки первого и второго типов - две стороны одной и той же медали, однако их последствия могут быть в корне отличны. Реализация рисков второго рода представляет несравнимо большую опасность. Ошибки второго рода создают ложную уверенность в правильности и надежности модели. Ситуацию усугубляет то, что ошибки второго рода более вероятны. Дело в том, что в условиях жесткой конкуренции и сжатых сроков, редкий разработчик будет подвергать сомнению и перепроверке модель, адекватность которой подтверждается экспериментально, затрачивая на это дополнительные денежные средства и временные ресурсы.

\section{1. Верификация и валидация газодинамического кода для численного моделирования высокоскоростных сжимаемых течений совершенного газа}

Ниже подробно рассматривается один из этапов многопланового процесса верификации компьютерного кода. В качестве объекта тестирования рассматривается программная реализация относительно простой газодинамической модели, которая является составным элементом интегрированных моделей сложных физико-химических процессов, разрабатываемых в ИПМех РАН. Компьютерный код основан на интегрировании двумерной системы уравнений Эйлера на неструктурированной сетке [2,20,21] с использованием метода установления на базе нестационарной схемы сквозного счета. Моделирование проводится без предварительного выделения поверхностей разрывов на основе хорошо известного метода расщепления по физическим процессам $[179,180]$ с применением неструктурированных сеточных моделей.

Использование данного метода на нерегулярных сетках позволяет получить общие характеристики потока сжимаемого газа для широкого диапазона скоростей, и дает возможность изучать картину течения со сложной конфигурацией ударных волн. 
Рассматривается класс двумерных задач течения химически нереагирующей, невязкой совершенной сжимаемой среды, которая описывается уравнениями Эйлера, в которых не учитывается вязкость и теплопроводность газа. Для столбца консервативных переменных $\mathbf{w}=(\rho, \rho u, \rho v, \rho E)^{T}$ система уравнений газовой динамики может быть записана в векторном виде

$$
\frac{\partial \mathbf{w}}{\partial t}+\frac{\partial \mathbf{F}^{\mathbf{x}}(\mathbf{w})}{\partial x}+\frac{\partial \mathbf{F}^{\mathbf{y}}(\mathbf{w})}{\partial y}=0,
$$

где $\mathbf{F}^{\mathbf{x}}=\left(\rho u, \rho u^{2}+p, \rho u v, \rho u E+p u\right)^{T}, \mathbf{F}^{\mathbf{y}}=\left(\rho v, \rho u v, \rho v^{2}+p, \rho v E+p v\right)^{T}-$ проекции вектора конвективного потока; $\rho$ - плотность; $p$ - давление; $u, v$ - компоненты вектора скорости; $E$ - удельная полная энергия газа. Система уравнений Эйлера используется совместно с уравнением состояния совершенного газа.

Основная идея метода состоит в расщеплении по физическим процессам исходной нестационарной системы уравнений (15). Стационарное решение задачи, если оно существует, получается в результате установления. Расчет каждого временного шага разбивается на несколько этапов. На первом этапе определяются промежуточные значения параметров потока без учета эффектов переноса. На втором этапе вычисляются эффекты переноса, учитывающие обмен между элементами - рассчитываются потоки массы через границы расчетных ячеек. На третьем этапе определяются в новый момент времени окончательные значения газодинамических параметров потока на основе законов сохранения массы, импульса и энергии для каждого элемента и всей системы в целом.

Для аппроксимации уравнений на каждом этапе применяются элементы метода конечных объемов. В рамках данного подхода определяются усредненные значения производных по контрольному объему (в качестве контрольного объема, например, рассматривается треугольная расчетная ячейка исходного сеточного разбиения).

$$
\begin{aligned}
\left\langle\frac{\partial f}{\partial \alpha}\right\rangle_{S_{i}} & =\frac{1}{S_{i}} \int_{S_{i}} \frac{\partial f}{\partial \alpha} \mathrm{d} S=\frac{1}{S_{i}} \int_{L_{i}} f \mathbf{i} \cdot \mathrm{d} \mathbf{L} \approx \frac{1}{S_{i}} \sum_{j=1}^{3} L_{i}^{j} n_{i, j}^{\alpha} f_{i}^{j}, \\
f & =(u, v, p), i=1 \ldots N, j=1 \ldots 3, \alpha=x, y
\end{aligned}
$$

Здесь $S_{i}$ - площадь $i$-го расчетного элемента; $L_{i}^{j}$ - длина $j$-го ребра $i$-го элемента; $n_{i, j}^{\alpha}-x$ или $y$ составляющая единичной нормали, задающая ориентацию $j$-го ребра $i$-го элемента.

Реализация метода расщепления по физическим процессам подразумевает решение большого количества однотипных подзадач по вычислению потоков через грани контрольных объемов (ребра треугольных элементов).

Рассматриваемая схема имеет большой запас устойчивости, которая обеспечивается наличием значительной аппроксимационной вязкости. При использовании классического варианта метода расщепления, обладающего первым порядком аппроксимации, на неортогональных сетках схемная вязкость приводит к сильной диффузии ударных волн. Бесконечно тонкие в невязком газе гидродинамические разрывы в численном решении приобретают толщину и физически необоснованно увеличивают область возмущенного течения. Последнее обстоятельство вносит погрешность решения, неустранимую простым измельчением ячеек, и может привести к неадекватному описанию динамики потока невязкого газа [19].

Для повышения порядка точности численной схемы с сохранением ее устойчивости, в рамках метода расщепления по физическим процессам, применялся метод аппроксимации потоков AUSM (Advection Upstream Splitting Method) [29,181], основанный на расщеплении вектора потоков искомых параметров на границах расчетных ячеек. Используемый подход обеспечивает аппроксимацию второго порядка точности по пространству (за исключением зон больших градиентов, где возможно снижение порядка аппроксимации до первого). 
Верификация газодинамической модели проводится на базе нескольких тестовых задач, описание и постановка которых приводится в стандарте [19]. Задачи формулируются для невязкого течения сжимаемого совершенного газа с постоянным показателем адиабаты $\gamma$ и молекулярным весом $M_{A}$ при отсутствии внешних массовых и поверхностных сил. Это позволяет проводить верификацию результатов численного моделирования путем сравнения с точными решениями и условно эталонными численными решениями, многократно подтвержденными разными авторами с использованием разнообразных численных схем [19].

В качестве первой задачи рассматривается процесс обтекания стенки с изломом (рис. 7). Равномерный сверхзвуковой поток натекает на острую кромку выпуклого излома плоской стенки и поворачивается на угол $\theta$. Возмущения, вносимые в поток изломом стенки, распространяются в виде центрированного в точке излома веера волн разрежения Прандтля - Майера $[35,182,183]$. Радиально расходящийся веер волн разделяет две области однородного течения. Постановка задачи представлена на рис. 7. Здесь схематически показана центрированная волна разрежения и две области равномерного течения, характеризующиеся набором параметров: давлением, плотностью и скоростью (числом Маха). Контур стенки задается двумя лучами, исходящими из одной точки и образующими развернутый угол $\left(180^{\circ}+\theta\right)$. Один из лучей совпадает с осью абсцисс прямоугольной декартовой системы координат, второй - расположен под осью. На верхней границе - прямой параллельной оси $x$, задаются условия открытой границы, на которую сносятся текущие параметры потока. На стенке ставятся условия непротекания (равенство нулю потоков массы и энергии по нормали к поверхности). Слева и справа расчетная область ограничена прямыми параллельными оси ординат.

Сверхзвуковой поток, направленный по оси $x$, втекает в расчетную область через левую границу, на которой задаются неизменные во времени параметры набегающего потока: давление $p_{\mathrm{I}}$, плотность $\rho_{\mathrm{I}}$ и скорость $V_{\mathrm{I}}$ или число Маха $\mathrm{M}_{\mathrm{I}}=V_{\mathrm{I}} / c_{\mathrm{I}}$. Для расчета скорости звука используется соотношение $c_{\mathrm{I}}=\sqrt{\gamma p_{\mathrm{I}} / \rho_{\mathrm{I}}}$. Входные параметры полностью определяют состояние газа $p_{\text {II }}, \rho_{\text {II }}, V_{\text {II }}\left(\mathrm{M}_{\text {II }}\right)$ во второй области за веером волн разрежения. Правая граница объявляется открытой. Для реализации течения Прандтля-Майера должно выполняться условие $\mathrm{M}_{\mathrm{I}} \geq 1$, при котором неизменно $\mathrm{M}_{\mathrm{II}} \geq \mathrm{M}_{\mathrm{I}}$. Величина угла разворота потока $\theta$ выбирается в пределах значений, обеспечивающих безотрывное обтекание стенки с изломом [182]. Конкретное положение границ области не влияет на решение, если они находятся на значительном расстоянии от кромки излома. Протяженность области интегрирования должна быть достаточно большой (относительно размера расчетных ячеек) для формирования обширных зон однородного течения (I и II) и хорошо развитого веера волн разрежения.

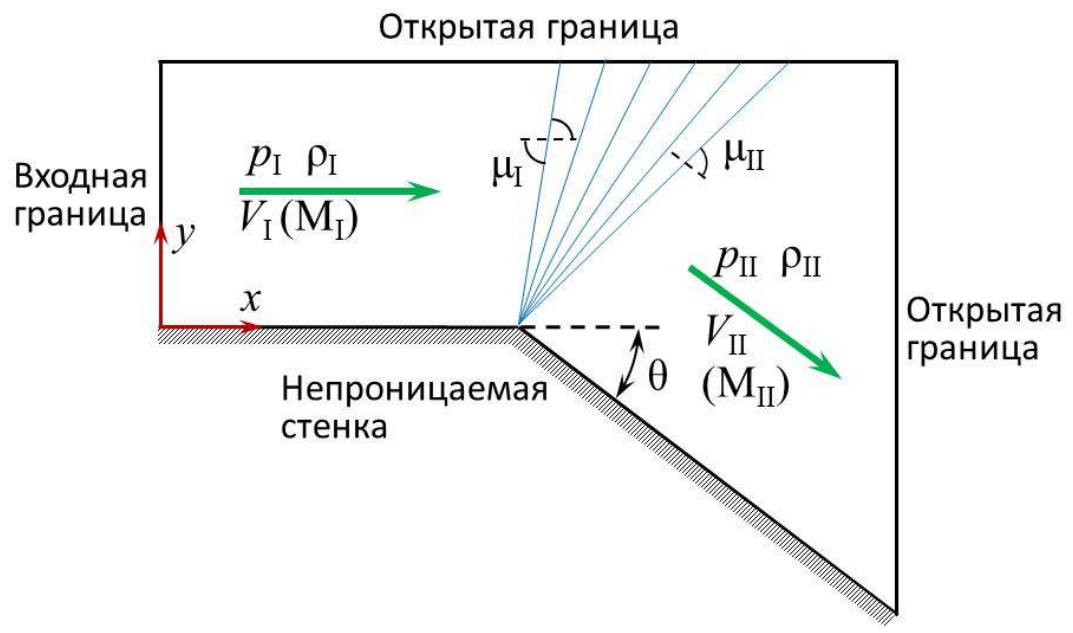

Рис. 7. Задача сверхзвукового обтекания стенки с изломом в двумерной постановке 
Рассматриваемая тестовая задача предназначена для верификации газодинамического кода, реализующего интегрирование двумерной системы уравнений Эйлера (15), и имеет точное аналитическое решение. Набор параметров, определяющих соответствие между точным решением и данными численного моделирования (так называемых метрик погрешности математической модели), должен включать все аспекты исследуемого процесса. Среди последних: расположение и конфигурация веера волн разрежения, изменение свойств потока при прохождении через веер волн разрежения, характеристики однородного течения за волной разрежения Прандтля-Майера. В частности, для параметров $V_{\text {II }}, p_{\text {II }}$ и $\rho_{\text {II }}$ должны выполняться изоэнтропические соотношения [32,183].

$$
\frac{\rho_{\mathrm{I}}}{\rho_{\mathrm{II}}}=\left(\frac{1+\frac{\gamma-1}{2} \mathrm{M}_{\mathrm{II}}^{2}}{1+\frac{\gamma-1}{2} \mathrm{M}_{\mathrm{I}}^{2}}\right)^{\frac{1}{\gamma-1}}, \quad \frac{p_{\mathrm{I}}}{p_{\mathrm{II}}}=\left(\frac{1+\frac{\gamma-1}{2} \mathrm{M}_{\mathrm{II}}^{2}}{1+\frac{\gamma-1}{2} \mathrm{M}_{\mathrm{I}}^{2}}\right)^{\frac{\gamma}{\gamma-1}}, \quad \frac{V_{\mathrm{I}}}{V_{\mathrm{II}}}=\frac{\mathrm{M}_{\mathrm{I}}}{\mathrm{M}_{\mathrm{II}}}\left(\frac{1+\frac{\gamma-1}{2} \mathrm{M}_{\mathrm{II}}^{2}}{1+\frac{\gamma-1}{2} \mathrm{M}_{\mathrm{I}}^{2}}\right)^{\frac{1}{2}}
$$

При этом вектор скорости $V_{\text {II }}$ должен быть параллелен направлению стенки за кромкой излома. Результат верификации можно считать положительным, только при условии высокой степени соответствия численного и аналитического решений. Расхождение не должно превышать 1-3\% даже на грубой сетке.

Реализующееся вблизи выпуклого излома сверхзвуковое течение представляет собой бесконечный набор волн разрежения (центрированный веер волн), в каждой из которых поток изоэнтропически поворачивается на малый угол (см. рис.7) в диапазоне от $\mu_{\mathrm{I}}=\arcsin \left(1 / \mathrm{M}_{\mathrm{I}}\right)$ до $\mu_{\mathrm{II}}=\arcsin \left(1 / \mathrm{M}_{\mathrm{II}}\right)$.

Для числа Маха в потоке за изломом $\mathrm{M}_{\mathrm{II}}$, числа Маха в набегающем потоке $\mathrm{M}_{\mathrm{I}}$ и угла поворота течения $\theta$ справедлива формула $[32,183]$

$$
\begin{gathered}
\theta=\sqrt{\frac{\gamma+1}{\gamma-1}} \cdot \arctan \left(\sqrt{\frac{\gamma-1}{\gamma+1}\left(\mathrm{M}_{\mathrm{II}}^{2}-1\right)}\right)-\arctan \left(\sqrt{\mathrm{M}_{\mathrm{II}}^{2}-1}\right)- \\
-\sqrt{\frac{\gamma+1}{\gamma-1}} \cdot \arctan \left(\sqrt{\frac{\gamma-1}{\gamma+1}\left(\mathrm{M}_{\mathrm{I}}^{2}-1\right)}\right)+\arctan \left(\sqrt{\mathrm{M}_{\mathrm{I}}^{2}-1}\right)
\end{gathered}
$$

Выше была дана общая математическая постановка задачи о сверхзвуковом течении около стенки с изломом. В качестве конкретного примера рассматривается случай:

$\gamma=1.4, \quad M_{A}=29 \times 10^{-3}$ кг $/$ моль $, \quad \mathrm{M}_{\mathrm{I}}=2, \quad \theta=10.0001^{\circ}, \quad \rho_{\mathrm{I}}=1.27540 \mathrm{\kappa г} / \mathrm{M}^{3}, \quad p_{\mathrm{I}}=10^{5}$ Па , $V_{\mathrm{I}}=\mathrm{M}_{\mathrm{I}} \cdot \sqrt{\gamma p_{\mathrm{I}} / \rho_{\mathrm{I}}}=662.630 \mathrm{~m} / \mathrm{c}$. Для данного варианта, с использованием зависимостей (17), (18), может быть получено точное решение:

$$
\begin{aligned}
& \mathrm{M}_{\mathrm{II}}=2.38489, \rho_{\mathrm{II}}=\rho_{\mathrm{I}} / 1.53675=0.82993 \kappa \Gamma / \mathrm{M}^{3}, p_{\mathrm{II}}=p_{\mathrm{I}} / 1.82493=5.47966 \times 10^{4} \text { Па }, \\
& V_{\mathrm{II}}=V_{\mathrm{I}} / 0.91387=725.084 \mathrm{M} / \mathrm{c}, \mu_{\mathrm{I}}=30.0000^{\circ}, \mu_{\mathrm{II}} \approx 24.7908^{\circ} .
\end{aligned}
$$

Результаты численного моделирования представлены на рис. 8-11 и помещены в табл. 2 .

Вычисления проводились на неструктурированных расчетных сетках различной подробности с применением численных схем первого и второго порядка точности по пространству. Разрешение сеточных моделей последовательно увеличивалось путем деления каждого ребра триангуляции на две части и последующего разбиения каждой расчетной ячейки на четыре треугольника (рис. 8). 


\section{Таблича 2}

Характеристики двух однородных областей, разделенных веером волн разрежения. Результаты моделирования, полученные с использованием схем первого и второго порядка на сетках различной размерности

\begin{tabular}{|c|c|c|c|c|c|c|c|}
\hline & \multicolumn{2}{|c|}{$E=16032$} & \multicolumn{2}{|c|}{$E=64128$} & \multicolumn{2}{|c|}{$E=256512$} & \multirow{2}{*}{$\begin{array}{c}\text { Точное } \\
\text { решение }\end{array}$} \\
\hline Порядок & 1-й & 2-й & 1-й & 2-й & 1-й & 2-й & \\
\hline $\mathrm{M}_{\mathrm{I}}$ & 2.00001 & 2.00001 & 2.00001 & 2.00001 & 2.00001 & 2.00001 & 2.00000 \\
\hline $\mathrm{M}_{\mathrm{II}}$ & 2.37703 & 2.38434 & 2.38022 & 2.38480 & 2.38216 & 2.38487 & 2.38489 \\
\hline$p_{\text {I }}$ & 100000 & 100000 & 100000 & 100000 & 100000 & 100000 & 100000 \\
\hline$p_{\text {II }}$ & 54858 & 54824 & 54830 & 54801 & 54815 & 54797 & 54796.6 \\
\hline$\rho_{\mathrm{I}}$ & 1.27541 & 1.27541 & 1.27541 & 1.27541 & 1.27541 & 1.27541 & 1.27540 \\
\hline$\rho_{\text {II }}$ & 0.82797 & 0.83014 & 0.82871 & 0.82996 & 0.82922 & 0.82994 & 0.82993 \\
\hline$V_{\mathrm{I}}$ & 662.630 & 662.630 & 662.630 & 662.630 & 662.630 & 662.630 & 662.630 \\
\hline$V_{\mathrm{II}}$ & 723.955 & 725.006 & 724.418 & 725.073 & 724.686 & 725.076 & 725.084 \\
\hline
\end{tabular}
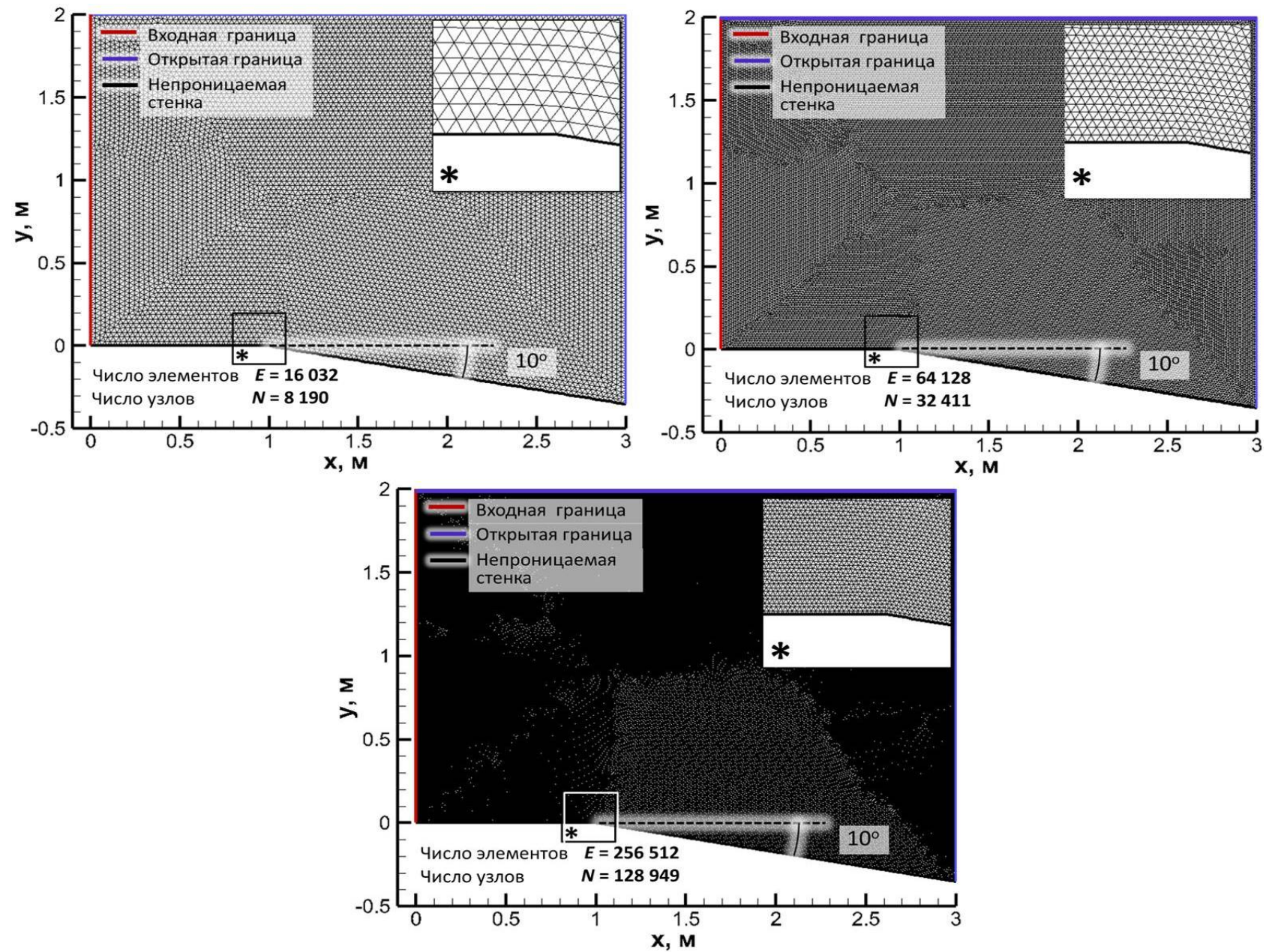

Рис. 8. Расчетная область с граничными условиями, дискретизированная с использованием различного количества ячеек. Разрешение неструктурированных сеток увеличивается путем последовательного четырехкратного увеличения числа треугольных элементов 
На рис. 9 показаны расчетные распределения чисел Маха вблизи стенки с изломом для различных численных схем и сеточных моделей. Из представленных данных видно, что качество разрешения особенностей структуры течения возрастает с увеличением подробности расчетных сеток и повышением порядка аппроксимации численного метода. В частности, изолинии поля чисел Маха становятся предельно близкими к прямым линиям. Ширина центрированной волны разрежения, возрастающая как функция расстояния до кромки излома, а также значения параметров потока за веером волн разрежения приближаются к теоретически предсказанным (см. табл. 2). В рассмотренном случае повышение порядка точности намного эффективней, чем сгущение расчетной сетки.
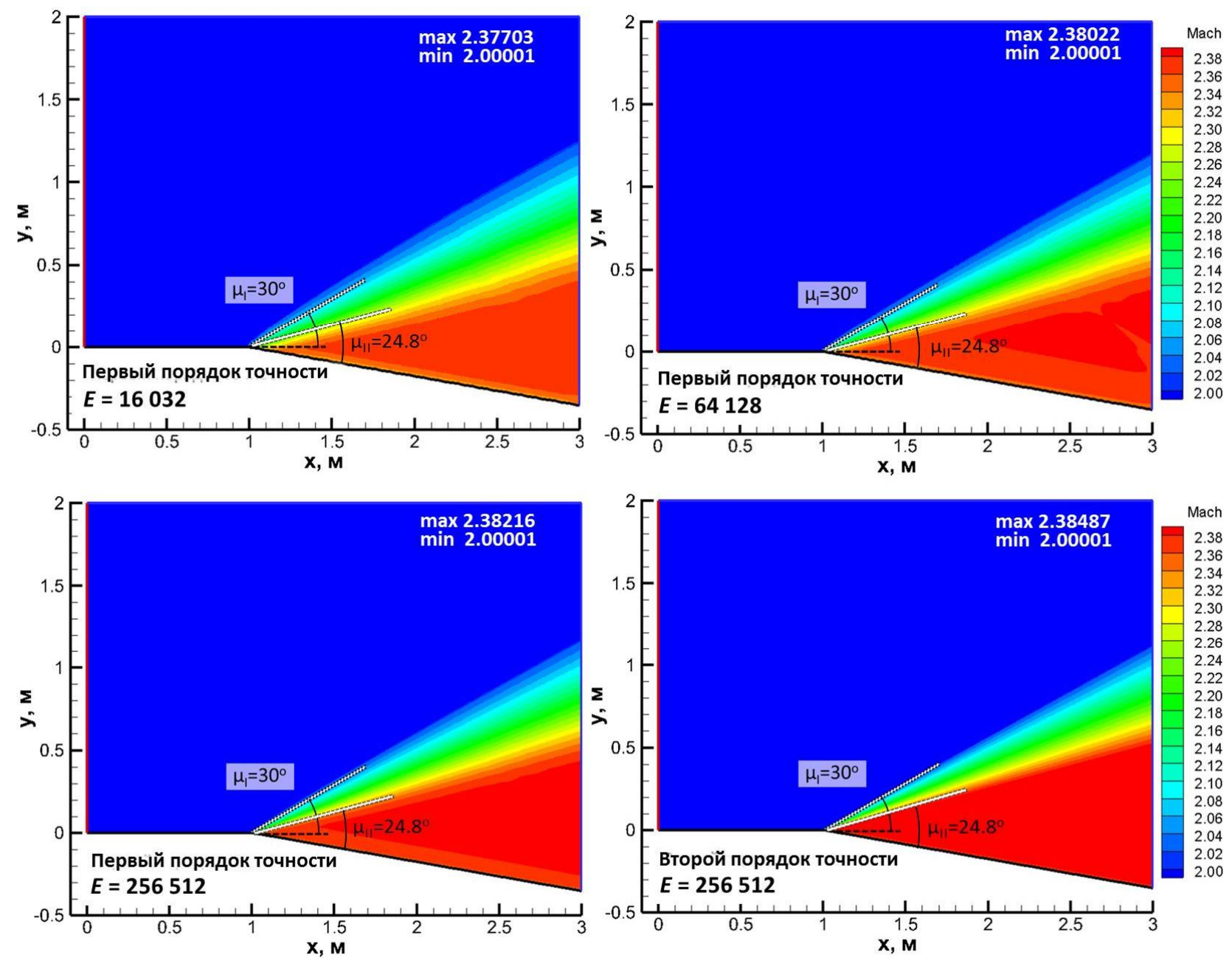

Рис. 9. Поля чисел Маха. Результаты вычислений, проведенных с первым и вторым порядком точности по пространству на неструктурированных сетках различной подробности

Исследуемое течение Прандтля-Майера является изоэнтропическим. Однако при отсутствии вязкости и теплопроводности в рассматриваемой модели условие постоянства энтропии будет нарушаться вследствие наличия схемной вязкости. Поведение (изменение) энтропии в волне разрежения может служить индикатором корректности кода и выявить степень искажения решения вызываемого искусственной вязкостью. На рис. 10 показан характер изменения энтропии во всем поле течения для численных решений, представленных на рис.9, а также остальных расчетных случаев. Возрастание энтропии, которое является следствием диссипации поступательной механической энергии, наблюдается в области, прилегающей к наклонному участку обтекаемой стенки. Область генерации энтропии расположена вниз по потоку от кромки излома и ограничена пристеночным слоем толщиной порядка десяти слоев расчетных ячеек. 



Рис. 10. Поля изменения удельной энтропии $\Delta S$, Дж/(кг $\mathrm{K})$ в расчетной области при течении внутри тупого угла. Результаты моделирования, проведенного с первым и вторым порядком точности по пространству на неструктурированных сетках различной подробности

Увеличение подробности сеточной модели приводит к уменьшению толщины области, однако максимальный прирост удельной энтропии почти не зависит от разрешения сетки. При переходе от первого порядка точности ко второму наибольшее изменение энтропии снижается от 24 Дж/(кг $\cdot \mathrm{K})$ до 17 Дж/(кг $\cdot \mathrm{K})$. Нарушение условия постоянства энтропии обусловлено наличием схемной вязкости, характерной для используемых численных методик. Изменение энтропии рассчитывалось из следующего соотношения:

$$
\Delta S=S-S_{\mathrm{I}}=\left(\frac{\gamma R}{\gamma-1}\right) \ln \left(\frac{T}{T_{\mathrm{I}}}\right)-R \ln \left(\frac{p}{p_{\mathrm{I}}}\right)
$$


Здесь $R=R_{0} / M_{A}$ - удельная газовая постоянная, равная отношению универсальной газовой постоянной $R_{0}=8.314$ Дж/(моль $\left.\cdot \mathrm{K}\right)$ и молярной массы газа $M_{A}$. Точным аналитическим решением поставленной задачи является полностью изоэнтропическое течение, поэтому любое увеличение энтропии - это паразитный численный эффект, который не имеет отношение к моделируемому физическому процессу.

На рис. 11 представлены распределения давления вдоль линии тока, которая начинается на входной границе в точке $y=0.25$ м. Профили были получены с использованием схем первого и второго порядков точности, а также сеток различной подробности. Рисунок показывает характер изменения параметра при прохождении веера волн разрежения. Из графиков видно, что центр расчетного профиля давления (на участке его падения) в веере волн разрежения не совпадает с центром профиля, соответствующего точному решению. Последнее можно объяснить неравнозначностью проявления эффектов численной диффузии в направлениях вверх и вниз по потоку. В остальном аналитическое решение воспроизводится достаточно точно даже на грубых неструктурированных сетках. При измельчении расчетных элементов и повышении порядка используемых численных схем точность решения монотонно возрастает, асимптотически приближаясь к точному решению. На основе полученных результатов можно сделать вывод о сеточной сходимости.
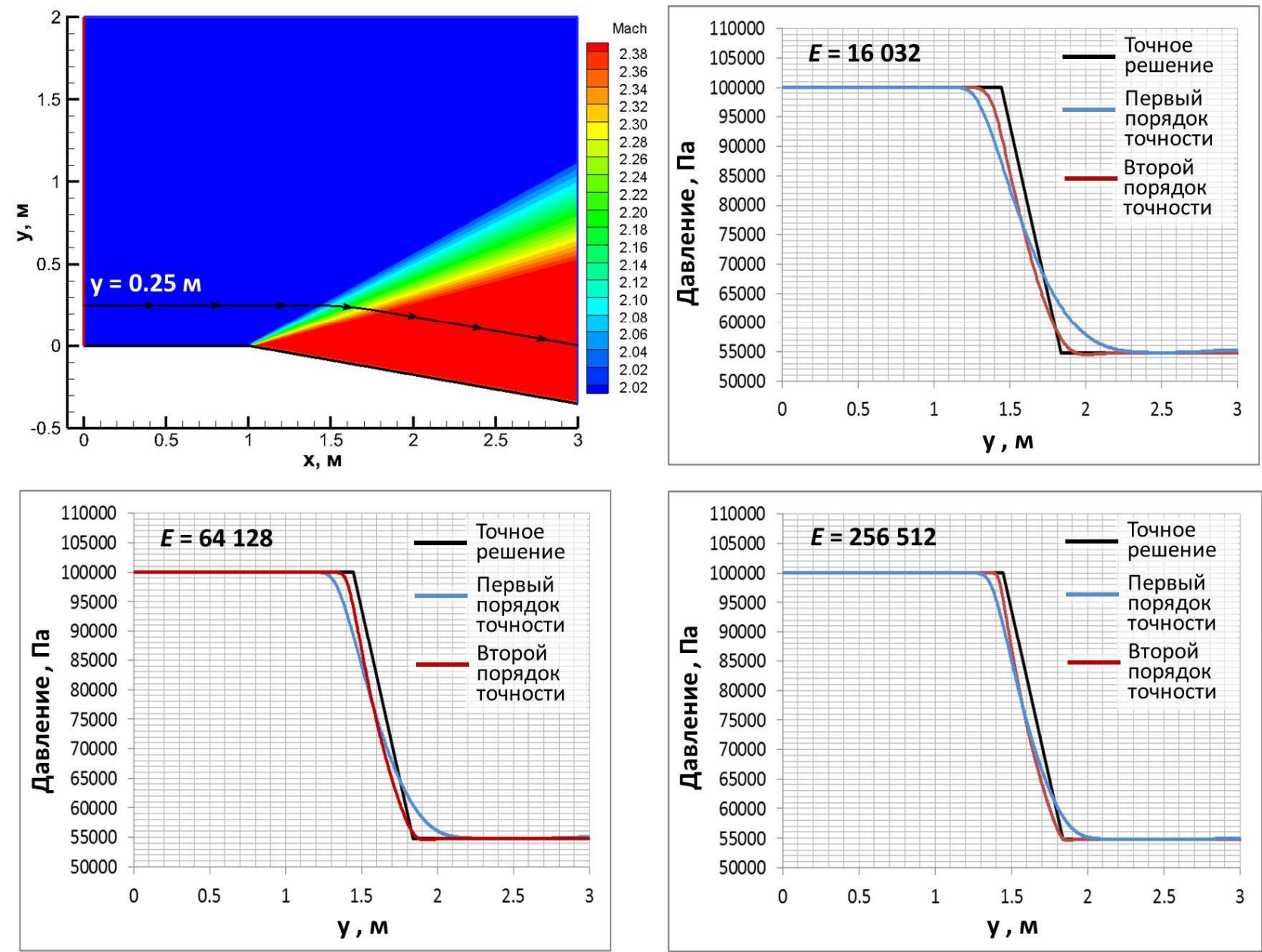

Рис. 11. Распределения давления вдоль линии тока, полученные на сетках различной подробности с использованием расчетных схем первого и второго порядка точности

Оценка степени сходимости итерационного процесса проводилась с использованием невязок - функций, характеризующих соответствие численного решения исходным дифференциальным уравнениям с определенными граничными условиями. В ходе итерационного 
процесса вычислений с первым порядком точности невязка монотонно убывала, а установившиеся значение данного критерия не превышало $10^{-12}$ (рекомендуемый предел для газодинамических вычислений с числами двойной точности) для каждого из четырех уравнений газовой динамики (15). Переход к схемам второго порядка отрицательно влиял на сходимость (особенно на самой подробной сетке), но значение соответствующего критерия, в любом случае, не превышало $10^{-6}$.

Для рассмотренного тестового случая валидация не требовалась, так как на этапе верификации исследуемой численной модели использовались точные аналитические решения.

В качестве второй верификационной задачи рассматривается продольное обтекание однородным сверхзвуковым потоком плоского клина с углом раствора $2 \cdot \theta$. Контур клина задается двумя лучами, исходящими из одной точки. Скорость потока параллельна биссектрисе угла раскрытия клина и направлена вдоль оси абсцисс [19]. При замене нулевой линии тока твердой стенкой поставленную задачу можно свести к задаче о течении газа внутри тупого угла (рис. 12). Таким образом, сверхзвуковой поток, параллельный стенке и оси $x$ на входе, в вершине угла меняет свое направление и двигается вдоль наклонного луча. В результате натекания на поверхность сжатия образуется косой скачек уплотнения, исходящий из вершины угла. Наклонная ударная волна разделяет две области однородного течения, которые характеризуются параметрами: давлением, плотностью, скоростью (числом Маха) $p_{\mathrm{I}}, \rho_{\mathrm{I}}$, $V_{\mathrm{I}}\left(\mathrm{M}_{\mathrm{I}}\right)$ и $p_{\mathrm{II}}, \rho_{\mathrm{II}}, V_{\mathrm{II}}\left(\mathrm{M}_{\mathrm{II}}\right)$ соответственно. Первый набор параметров определяет постоянные во времени свойства набегающего потока, втекающего через левую границу расчетной области. Рисунок дает представление о граничных условиях, а также структуре течения.

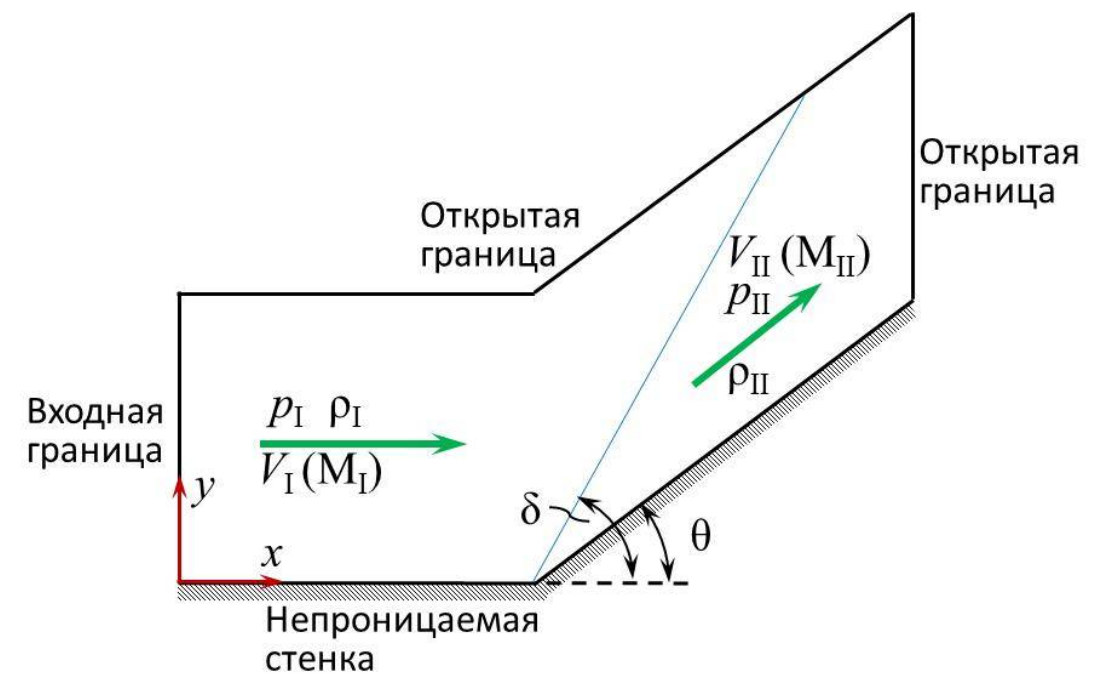

Рис. 12. Задача плоского сверхзвукового течения внутри тупого угла

Расчетная область снизу ограничена стенкой, на которой определяются условия непротекания (равенство нулю потоков массы и энергии по нормали к поверхности). Верхняя граница задается в виде ломаной линии, состоящей из отрезка, направленного вдоль оси абсцисс и сегмента, параллельного наклонному участку обтекаемой поверхности (см. рис. 12). Слева на входе и справа на выходе расчетная область ограничена прямыми, параллельными оси ординат [19]. На верхней и выходной границах ставятся условия открытой границы, на которую сносятся текущие параметры потока. Неизменные параметры потока, втекающего через входную границу $\left(p_{\mathrm{I}}, \rho_{\mathrm{I}}, V_{\mathrm{I}}\right.$ или $\left.\mathrm{M}_{\mathrm{I}}\right)$ и геометрия расчетной области (угол наклона стенки $\theta$ ) полностью определяют структуру течения (угол наклона ударной волны $\delta$ ), а также состояние газа $\left(p_{\mathrm{II}}, \rho_{\mathrm{II}}, V_{\mathrm{II}}\right.$ или $\left.\mathrm{M}_{\mathrm{II}}\right)$ во второй области за косым скачком. Для формирования наклонной ударной волны необходимо выполнение следующего условия: $\mathrm{M}_{\mathrm{I}} \cdot \sin \delta \geq 1$. Рас- 
стояние от вершины тупого угла до границ расчетной области не оказывает влияния на результат моделирования, однако выбирается достаточным для формирования обширных зон однородного течения.

Рассматриваемая тестовая задача также предназначена для верификации двумерной газодинамической модели, основанной на интегрировании системы уравнений Эйлера (15). Результат верификации можно считать положительным, только при условии высокой степени соответствия численного и аналитического решений. Допустимое различие не должно превышать 1-3\% даже на грубой сетке.

В ходе тестирования проводилось сопоставление результатов моделирования с точным аналитическим решением по ряду параметров, таких как угол наклона ударной волны $\delta$, характеристики однородного потока за косым скачком $V_{\mathrm{II}}, p_{\mathrm{II}}$ и $\rho_{\mathrm{II}}$.

Точное решение описывается соотношениями [184]

$$
\begin{aligned}
& \mathrm{M}_{\mathrm{II}}^{2}=\frac{1+\frac{\gamma-1}{2} \mathrm{M}_{\mathrm{I}}^{2}}{\gamma \cdot \mathrm{M}_{\mathrm{I}}^{2} \sin ^{2} \delta-\frac{\gamma-1}{2}}+\frac{\mathrm{M}_{\mathrm{I}}^{2} \cos ^{2} \delta}{1+\frac{\gamma-1}{2} \mathrm{M}_{\mathrm{I}}^{2} \sin ^{2} \delta} ; \\
& \frac{1}{\tan \theta}=\left[\left(\frac{\gamma+1}{2}\right)\left(\frac{\mathrm{M}_{\mathrm{I}}^{2}}{\mathrm{M}_{\mathrm{I}}^{2} \sin ^{2} \delta-1}\right)-1\right] \tan \delta \text {; } \\
& \frac{p_{\mathrm{II}}}{p_{\mathrm{I}}}=\left(\frac{2 \gamma}{\gamma+1}\right) \mathrm{M}_{\mathrm{I}}^{2} \sin ^{2} \delta-\left(\frac{\gamma-1}{\gamma+1}\right) \text {; } \\
& \frac{\rho_{\mathrm{II}}}{\rho_{\mathrm{I}}}=\frac{(\gamma+1) \mathrm{M}_{\mathrm{I}}^{2} \sin ^{2} \delta}{2+(\gamma+1) \mathrm{M}_{\mathrm{I}}^{2} \sin ^{2} \delta} \text {; } \\
& \frac{V_{\mathrm{II}}}{V_{\mathrm{I}}}=\frac{\sin \delta}{\sin (\delta-\theta)}\left[\frac{2}{(\gamma+1) \mathrm{M}_{\mathrm{I}}^{2} \sin ^{2} \delta}+\left(\frac{\gamma-1}{\gamma+1}\right)\right]
\end{aligned}
$$

При этом вектор скорости $V_{\text {II }}$ должен быть параллелен наклонному участку обтекаемой поверхности за вершиной тупого угла.

Для полной постановки задачи были выбраны следующие геометрические параметры расчетной области и свойства набегающего потока:

$\gamma=1.4, \quad M_{A}=29 \times 10^{-3}$ кг моль $, \quad \mathrm{M}_{\mathrm{I}}=3, \quad \theta=15.0000^{\circ}, \quad \rho_{\mathrm{I}}=1.27540 \kappa \Gamma / \mathrm{M}^{3}, \quad p_{\mathrm{I}}=10^{5} \Pi$ Па $V_{\mathrm{I}}=\mathrm{M}_{\mathrm{I}} \cdot \sqrt{\gamma p_{\mathrm{I}} / \rho_{\mathrm{I}}}=993.944 \mathrm{~m} / \mathrm{c}$. Для данного варианта, с использованием зависимостей (20) может быть получено точное решение:

$\mathrm{M}_{\mathrm{II}}=2.25490, \delta=32.2404^{\circ}, p_{\mathrm{II}}=2.82156 \cdot p_{\mathrm{I}}=282156$ Па, $\rho_{\mathrm{II}}=2.03245 \cdot \rho_{\mathrm{I}}=2.59219 \kappa \Gamma / \mathrm{M}^{3}$, $V_{\text {II }}=0.885608 \cdot V_{\mathrm{I}}=880.245 \mathrm{M} / \mathrm{c}$.

Вычисления выполнялись на неструктурированных сетках различной размерности с использованием численных схем первого и второго порядка точности по пространству. Число элементов сеточных моделей последовательно увеличивалось путем деления каждого ребра триангуляции на две части и последующего разбиения каждой расчетной ячейки на четыре треугольника (рис. 13).

Расчетные данные и результаты их сопоставления с точным решением помещены в табл. 3, а также представлены на рис. 14-16. Здесь отчетливо видны проявления эффектов численной диффузии в виде размытия фронта наклонной ударной волны. Некоторое искажение решения наблюдается в окрестности пересечения фронта с обтекаемой поверхностью (в вершине тупого угла) и выходной границей. С ростом порядка аппроксимации численных 
схем и увеличением разрешения сеточных моделей ширина размытия монотонно убывает. При этом теоретически предсказанные параметры потока за скачком уплотнения воспроизводятся с высокой степенью точности (на достаточном удалении от фронта ударной волны и обтекаемой поверхности) даже на относительно грубых сетках (табл.3).
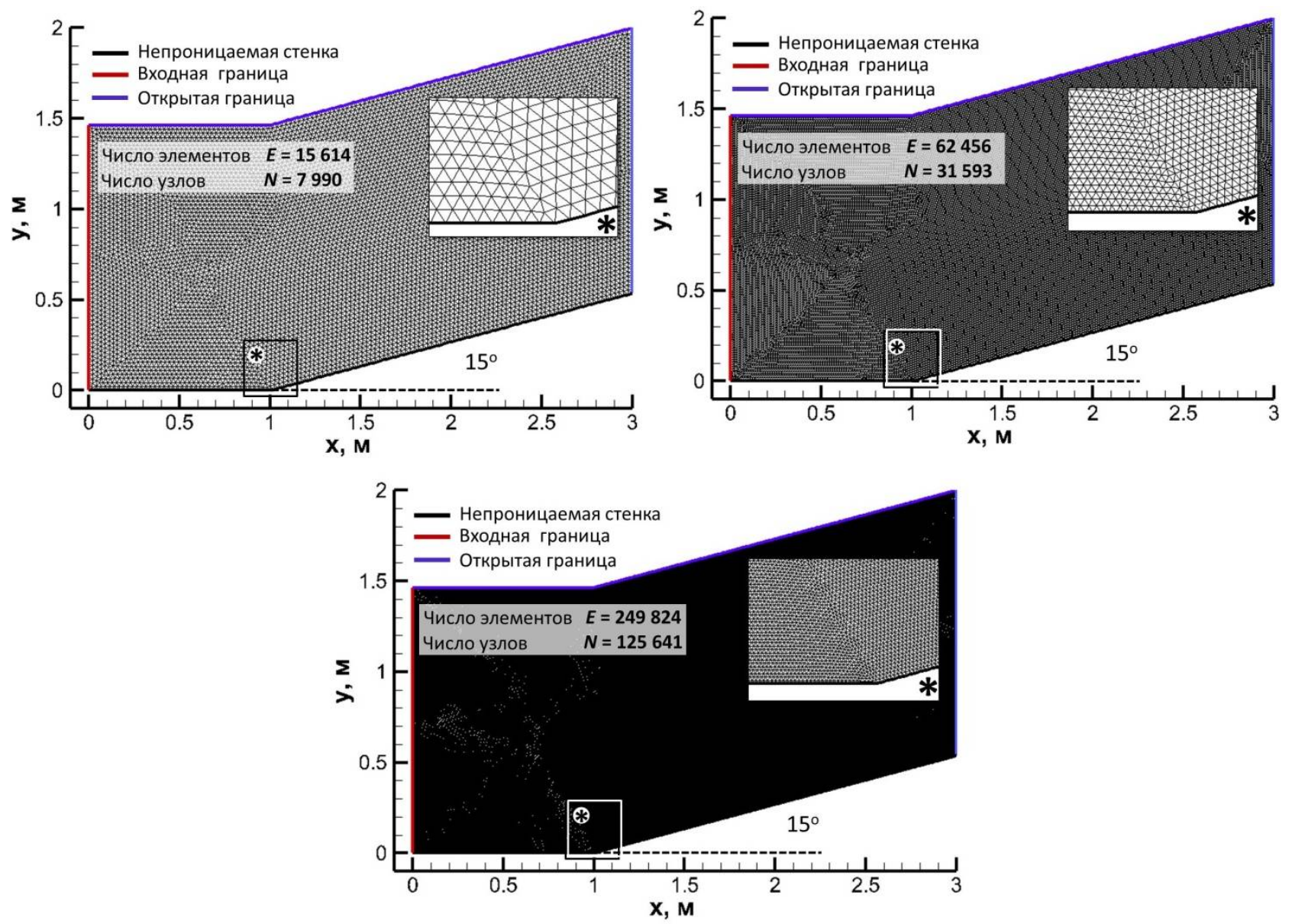

Рис. 13. Сеточные модели различной подробности с граничными условиями для моделирования течения внутри тупого угла. Разрешение неструктурированных сеток увеличивается путем последовательного разбиения каждого треугольного элемента на четыре ячейки

Таблийа 3

Параметры потока в однородных областях перед и за фронтом косой ударной волны. Результаты моделирования, полученные с использованием схем первого и второго порядка на сетках различной размерности

\begin{tabular}{|c|c|c|c|c|c|c|c|}
\hline & \multicolumn{2}{|c|}{$E=15614$} & \multicolumn{2}{c|}{$E=62456$} & \multicolumn{2}{c|}{$E=249860$} & \multirow{2}{*}{$\begin{array}{c}\text { Точное } \\
\text { решение }\end{array}$} \\
\cline { 1 - 6 } Порядок & 1 -й & 2 -й & 1 -й & 2-й & 1 -й & 2-й & \\
\hline $\mathrm{M}_{\mathrm{I}}$ & 3.00000 & 3.00000 & 3.00000 & 3.00000 & 3.00000 & 3.00000 & 3.00000 \\
\hline $\mathrm{M}_{\text {II }}$ & 2.24773 & 2.25408 & 2.25348 & 2.25507 & 2.25517 & 2.25487 & 2.25490 \\
\hline$p_{\text {I }}$ & 100000 & 100000 & 100000 & 100000 & 100000 & 100000 & 100000 \\
\hline$p_{\text {II }}$ & 282134 & 282219 & 282217 & 282114 & 282169 & 282160 & 282156 \\
\hline$\rho_{\text {I }}$ & 1.27540 & 1.27540 & 1.27540 & 1.27540 & 1.2754 & 1.27540 & 1.27540 \\
\hline$\rho_{\text {II }}$ & 2.58369 & 2.59178 & 2.59068 & 2.59241 & 2.59207 & 2.59220 & 2.59219 \\
\hline$V_{\text {I }}$ & 993.944 & 993.944 & 993.944 & 993.944 & 993.944 & 993.944 & 993.944 \\
\hline$V_{\text {II }}$ & 878.852 & 880.091 & 880.040 & 880.206 & 880.389 & 880.236 & 880.245 \\
\hline
\end{tabular}


На рис. 14 показаны поля чисел Маха, полученные на сетках различной подробности с использованием схем первого и второго порядков точности для скорости набегающего потока $\mathrm{M}=3$. Представленные данные демонстрируют хорошее согласование с аналитическим решением по структуре поток

На всех рисунках видны две однородные области течения, разделенные косым скачком. Качество разрешения указанных структур монотонно возрастает с увеличением числа расчетных элементов и при повышении порядка точности численных схем. Искажения численного решения вблизи вершины тупого угла за фронтом ударной волны, вызванные проявлением схемной вязкости, дают заниженные значения чисел Маха за скачком. На достаточном отдалении от кромки излома числа Маха приближаются к теоретически предсказанной величине $\left(\mathrm{M}_{\mathrm{II}}=2.25490\right)$.
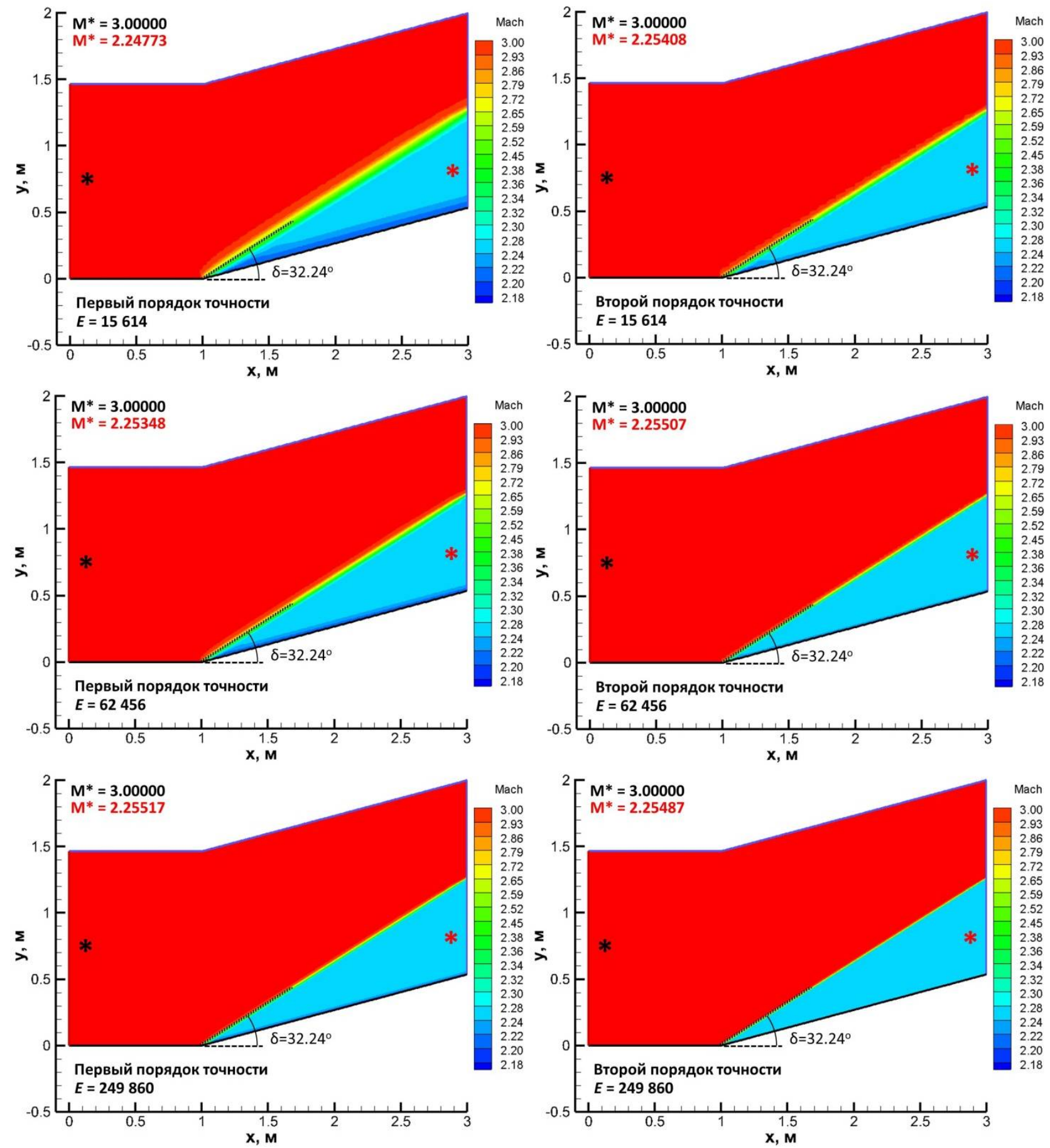

Рис. 14. Поля чисел Маха. Результаты вычислений, проведенных с первым и вторым порядком точности по пространству на неструктурированных сетках различной подробности 
Соответствующее изменение удельной энтропии потока в расчетной области для рассмотренных случаев представлено на рис. 15. Течения со скачками уплотнения не являются изоэнтропическими. Из точного решения прирост энтропии на фронте ударной волны составляет

$$
\left.\Delta S=S_{\mathrm{II}}-S_{\mathrm{I}}=\left(\frac{\gamma R}{\gamma-1}\right) \ln \left(\frac{p_{\mathrm{II}} / R \rho_{\mathrm{II}}}{p_{\mathrm{I}} / R \rho_{\mathrm{I}}}\right)-R \ln \left(\frac{p_{\mathrm{II}}}{p_{\mathrm{I}}}\right)=31.79 \text { Дж/(кГ } \cdot \mathrm{K}\right)
$$
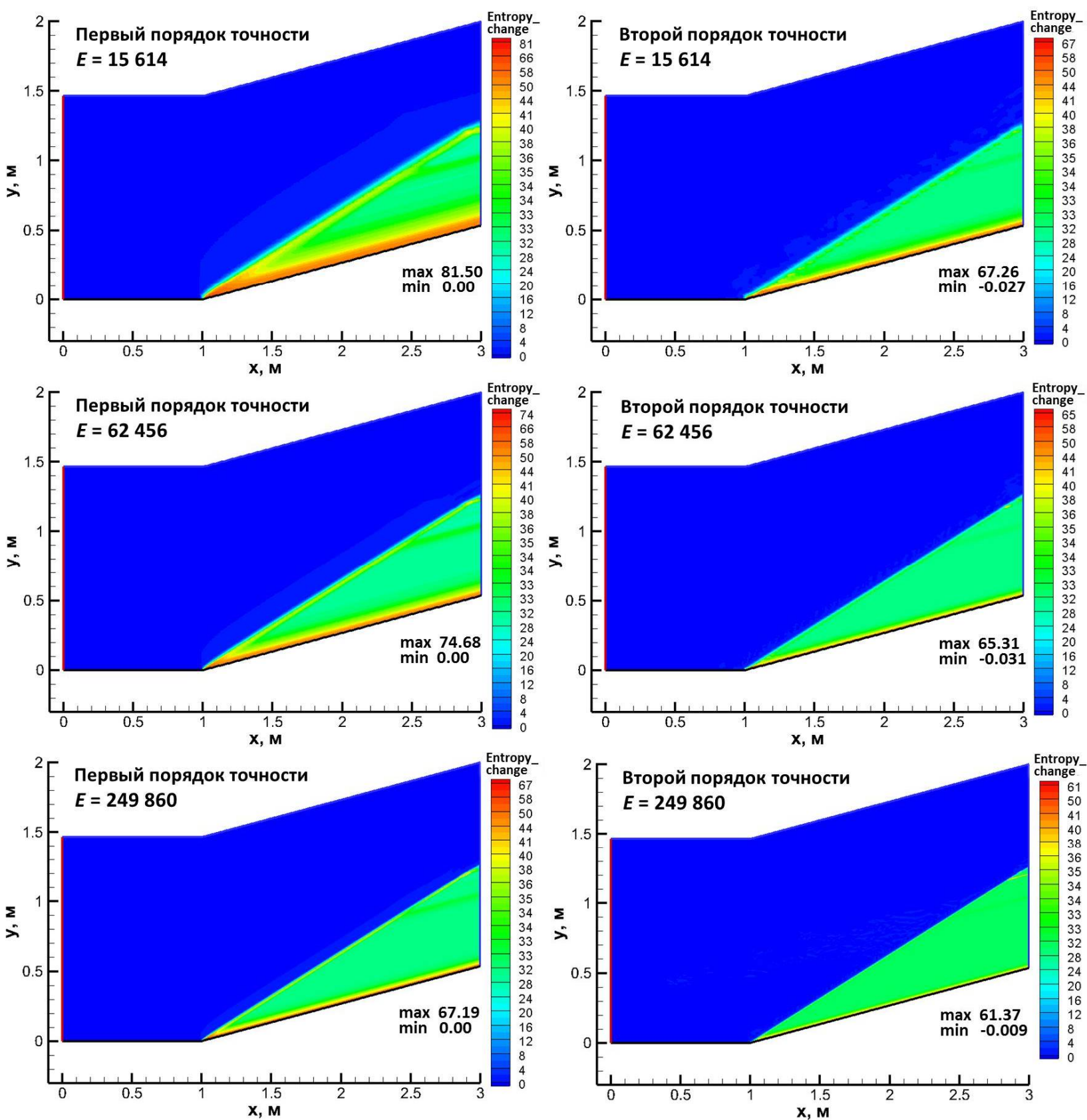

Рис.15. Характер изменения удельной энтропии $\Delta S$, Дж/(кг К) в расчетной области при течении внутри тупого угла. Результаты моделирования, проведенного с первым и вторым порядком точности по пространству на неструктурированных сетках различной подробности

Из рис. 15 видно, что ударная волна генерирует однородный скачек энтропии вдоль ее фронта, который количественно согласуется с аналитическим решением. На представленных иллюстрациях хорошо заметны проявления схемной диссипации (особенно при использова- 
нии первого порядка аппроксимации), которая вызывает дополнительное возрастание энтропии вблизи разрыва, а также ее локальное производство около вершины тупого угла и в области пересечения скачка с выходной границей. Искажение решения у кромки излома обтекаемой поверхности сносится вниз по течению, повышая значения энтропии в пристеночном слое. Указанные численные эффекты накладываются на физический скачек параметра на ударной волне, приводя к значительному суммарному приросту энтропии во всей однородной области течения за ее фронтом.

При повышении порядка аппроксимации численного метода до второго, прирост численно сгенерированной энтропии на расчетной сетке той же подробности снижается на 20 30 \%. При этом области локального производства энтропии (окрестность кромки излома обтекаемой поверхности и узкий пристеночный слой, истекающий из вершины тупого угла) уменьшаются в размерах. Аналогичные эффекты наблюдаются при увлечении подробности сеточных моделей. Небольшой отрицательный прирост энтропии (см. рис. 15, справа), возможно, обусловлен неустойчивостями, характерными для численных схем второго порядка точности.

Рис. 16 демонстрирует степень соответствия численных данных, полученных с использованием схем первого и второго порядка аппроксимации на сетках различной размерности, аналитическому решению. Результаты представлены в виде линейных графиков, отражающих изменение давления вдоль линии тока $y=0.25$ м при пересечении фронта косого скачка.
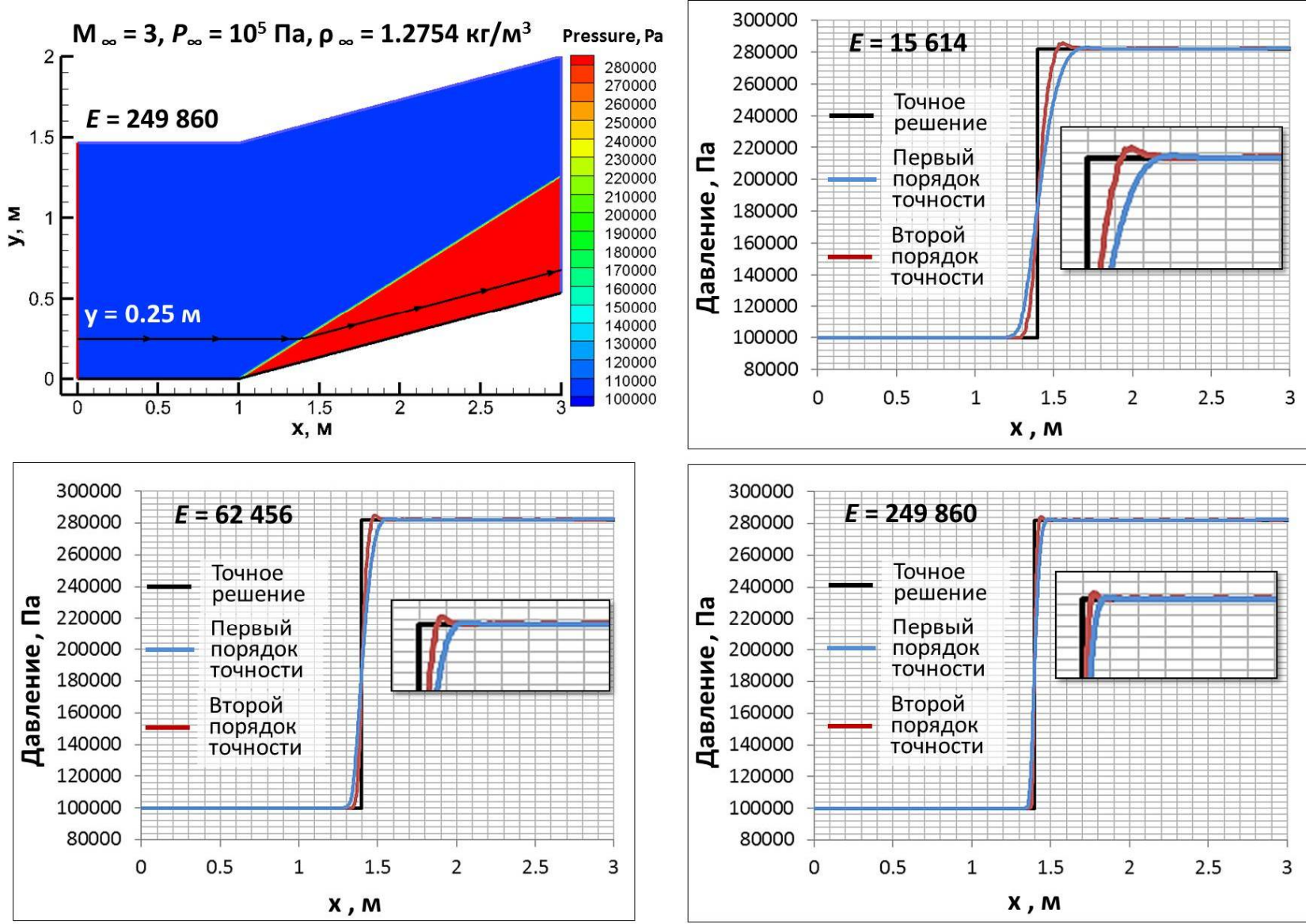

Рис. 16. Распределения давления вдоль линии тока $y=0.25$ м, полученные на сетках различной подробности с использованием расчетных схем первого и второго порядка точности

Переход на второй порядок аппроксимации обеспечивает точность, которая превосходит точность решения, полученного на треугольной сетке с учетверенным числом расчетных 
ячеек. С другой стороны, повышение порядка численной схемы вызывает осцилляции решения (особенно при увеличении подробности сеточной модели), что хорошо заметно на линейных графиках давления в однородной области за скачком уплотнения (см. рис. 16). При возрастании количества треугольных элементов численное решение асимптотически приближается к точному, что говорит о достижении сеточной сходимости.

Во всех расчетных случаях была достигнута высокая степень итерационной сходимости. В ходе вычислений с применением схем первого порядка значения невязок снижались на двенадцать порядков (рекомендуемый предел для газодинамических вычислений с числами двойной точности) для каждого из четырех уравнений газовой динамики (15). При расчетах на наиболее подробных сетках с использованием схем второго порядка наблюдалось возникновение неустойчивостей и ухудшение сходимости, однако значения невязок не превышали $10^{-4}$.

Для рассмотренного класса тестовых задач верификация может проводиться на базе точных решений, и не требует последующей валидации.

Изложенная технология валидации для тестируемого газодинамического кода реализуется на примере валидационной задачи внешнего обтекания крылового профиля NACA0012 с использованием экспериментальных данных [185], полученных в криогенной трансзвуковой аэродинамической трубе исследовательского центра НАСА Лэнгли. В аэродинамической установке были созданы условия для продувки модели профиля (крыла бесконечного размаха), обеспечивающие двумерность и однородность поля течения в рабочей части, что позволило максимально точно воспроизвести поведение двумерной численной модели. В работе [185] приводятся результаты экспериментальных исследований плоского течения около профиля NACA0012 в виде распределенных и интегральных аэродинамических характеристик поверхности крыла в широком диапазоне параметров набегающего потока.

Моделировалось двумерное течение невязкого сжимаемого совершенного газа около конфигурации NACA0012. В качестве примера рассматривался трансзвуковой режим обтекания со скоростью, соответствующей $\mathrm{M}_{\infty}=0.8$ под углом атаки $\alpha=1.25^{\circ}$.

Классическая геометрия данного крылового профиля задается уравнением [186]

$$
y= \pm 0.6\left(0.2969 \sqrt{x}-0.1260 x-0.3516 x^{2}+0.2843 x^{3}-0.1015 x^{4}\right)
$$

Соотношение (22) определяет симметричное крыло с нулевой толщиной задней кромки и длиной хорды $c_{0}=1.008930411$, поэтому для построения обтекаемой поверхности используется модифицированная формула, описывающая уменьшенную в $c_{0}=1.008930411$ раз модель крыла

$$
\bar{y} \cdot c_{0}= \pm 0.6 \cdot\left(\begin{array}{l}
0.2969 \cdot \sqrt{c_{0}} \cdot \sqrt{\bar{x}}-0.1260 \cdot c_{0} \cdot \bar{x}-0.3516 \cdot c_{0}^{2} \cdot \bar{x}^{2}+ \\
+0.2843 \cdot c_{0}^{3} \cdot \bar{x}^{3}-0.1015 \cdot c_{0}^{4} \cdot \bar{x}^{4}
\end{array}\right), \bar{x} \in[0,1],
$$

где $\bar{y}=y / c_{0}, \bar{x}=x / c_{0}$.

Окончательно получаем

$$
\bar{y}= \pm 0.594689181 \cdot\left(\begin{array}{l}
0.298222773 \sqrt{\bar{x}}-0.127125232 \bar{x}-0.357907906 \bar{x}^{2}+ \\
+0.291984971 \bar{x}^{3}-0.105174606 \bar{x}^{4}
\end{array}\right)
$$

Конфигурация профиля представлена на рис. 17.

Внешняя граница расчетной области составлена из сторон прямоугольника и дуги окружности радиусом 500 хорд с центром, находящимся на середине хорды (рис. 18). На удаленных внешних границах задаются граничные условия дальнего поля. Обтекаемая поверхность представляет собой непроницаемую твердую стенку, на которой ставится граничное условие проскальзывания. Для моделирования применялись неструктурированные сетки с 
различным числом треугольных элементов. Последовательное сгущение сеток проводилось методом деления каждой ячейки триангуляции на четыре треугольника (рис. 18).

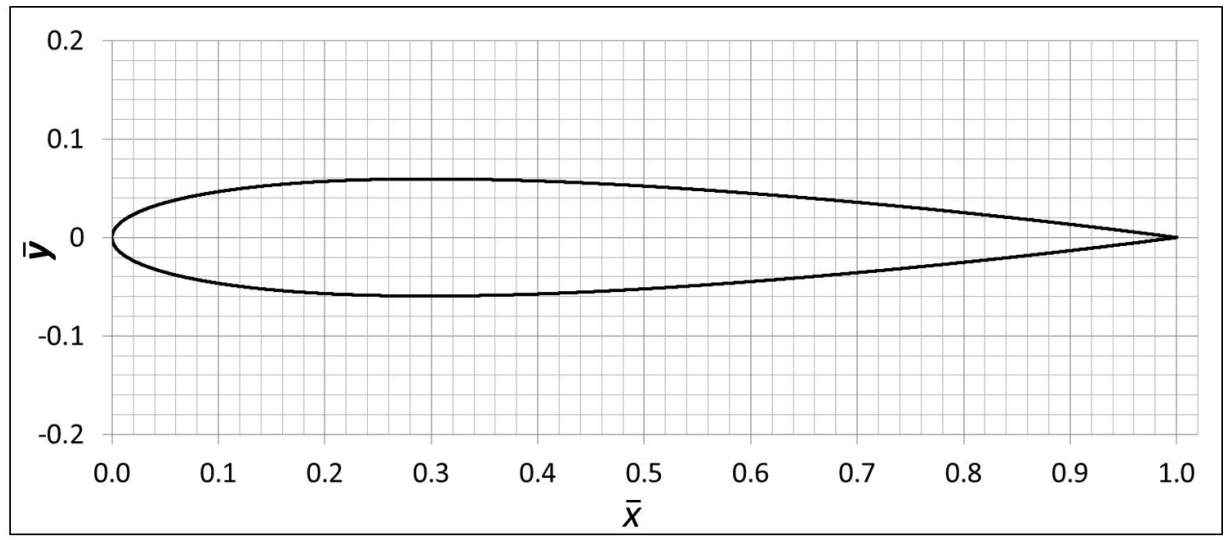

Рис. 17. Крыловой профиль NACA0012

В расчетах использовались параметры набегающего потока совершенного невязкого сжимаемого газа $(\gamma=1.4)$, которые соответствовали условиям серии экспериментов [185]. Исходные данные для численного моделирования (табл.4) рассчитывались из следующих соотношений:

$$
\begin{gathered}
p_{\infty}=\frac{p_{t}}{\left(1+\frac{\gamma-1}{2} \cdot \mathrm{M}_{\infty}^{2}\right)^{\frac{\gamma}{\gamma-1}}}, \quad T_{\infty}=\frac{T_{t}}{1+\frac{\gamma-1}{2} \cdot \mathrm{M}_{\infty}^{2}}, \quad \rho_{\infty}=\frac{p_{\infty}}{R \cdot T_{\infty}}, \\
c_{36}=\sqrt{\gamma R T_{\infty}}, \quad V_{\infty}=\mathrm{M}_{\infty} \cdot c_{36}
\end{gathered}
$$

Таблийа 4

Условия валидирующего эксперимента [185], исходные данные для расчета и свойства среды

\begin{tabular}{|c|c|}
\hline Параметры & Эксперимент \\
\hline Хорда крылового профиля $c, \mathrm{~m}$ & 0.1524 \\
\hline Расположение центра тяжести & $0.25 c$ \\
$-\begin{array}{c}\text { расстояние от носка вдоль оси } x \\
\text { - расстояние от носка вдоль оси } y\end{array}$ & 0 \\
\hline Число Маха $\mathrm{M}_{\infty}$ & 0.7980 \\
\hline Угол атаки $\alpha$ & 1.2516 \\
\hline Давление торможения $p_{t}$, Па & 165075.72 \\
\hline Температура торможения $T_{t}, \mathrm{~K}$ & 195.7614 \\
\hline & Численное моделирование \\
\hline Молярная масса $M_{A}, \mathrm{\kappa г} /$ моль & $29 \times 10^{-3}$ \\
\hline Удельная газовая постоянная $R$, Дж/(кг·К) & 286.7 \\
\hline Статическое давление $p_{\infty}$, Па & 108508.30 \\
\hline Статическая температура $T_{\infty}, \mathrm{K}$ & 173.6457 \\
\hline Плотность $\rho_{\infty}$, кг/м ${ }^{3}$ & 2.179650 \\
\hline Скорость звука $c_{3 в}, \mathrm{~m} / \mathrm{c}$ & 263.9989 \\
\hline Скорость потока $V_{\infty}, \mathrm{M} / \mathrm{c}$ & 210.6711 \\
\hline
\end{tabular}



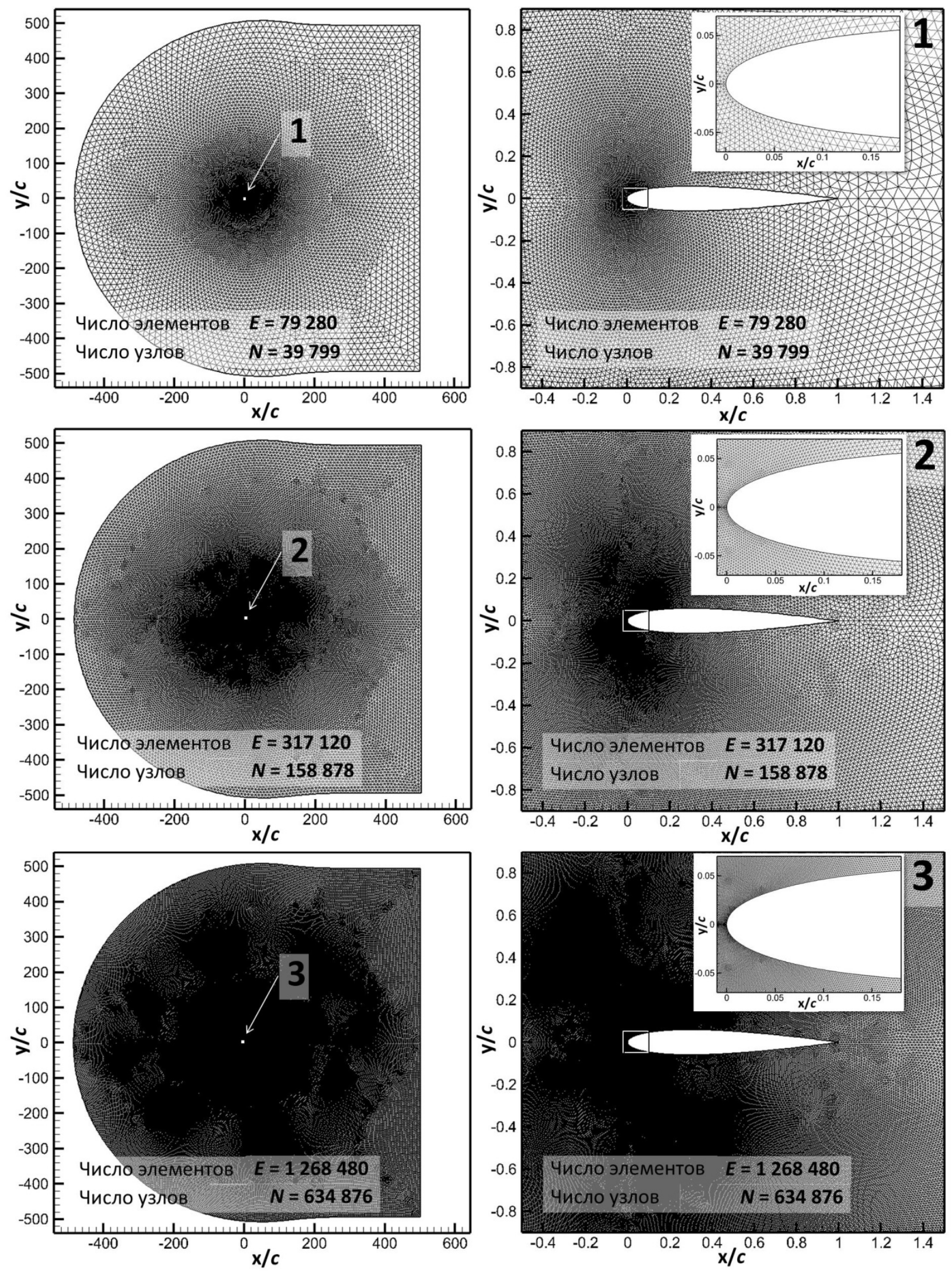

Рис. 18. Расчетная область с сетками различной размерности при последовательном увеличении числа ячеек в четыре раза

В начальный момент времени во всей области задавалось однородное поле течения с параметрами набегающего потока. 
В качестве валидационных критериев были выбраны распределенные и интегральные аэродинамические характеристики, доступные для экспериментального измерения [185].

Результаты численного моделирования представлены на рис. 19, 20 в виде полей чисел Маха и давления вблизи обтекаемого тела, а также линейных распределений коэффициентов давления $C_{P}$ по поверхности крыла. Полученные значения интегральных аэродинамических характеристик профиля NACA0012, таких как коэффициенты аэродинамических сил $C_{L}, C_{D}$, помещены в табл. 5.

Таблиия 5

\begin{tabular}{|c|c|c|c|c|}
\hline & \multicolumn{3}{|c|}{ Численное моделирование } & \multirow{2}{*}{$\begin{array}{c}\text { Экспериментальные } \\
\text { данные } \\
\end{array}$} \\
\hline & $E=79280$ & $E=317120$ & $E=1268480$ & \\
\hline $\begin{array}{l}\text { Коэффициент подъемной } \\
\text { силы, } C_{L}\end{array}$ & 0.3417 & 0.3466 & 0.3507 & 0.3529 \\
\hline $\begin{array}{l}\text { Коэффициент силы лобо- } \\
\text { вого сопротивления, } C_{D}\end{array}$ & 0.02374 & 0.02332 & 0.02298 & 0.02275 \\
\hline
\end{tabular}

Рис. 19, на котором представлены изолинии чисел Маха для сеток различной размерности, дает общее представление о структуре течения. Качество разрешения последней возрастает с увеличением подробности сеточных моделей.

Значения коэффициентов давления и аэродинамических сил вычислялись из соотношений

$$
C_{P}=\frac{p-p_{\infty}}{0.5 \rho_{\infty} V_{\infty}^{2}}, \quad C_{L}=\frac{F_{y}}{0.5 \rho_{\infty} V_{\infty}^{2} \cdot S_{\text {ref }}}, \quad C_{D}=\frac{F_{x}}{0.5 \rho_{\infty} V_{\infty}^{2} \cdot S_{\text {ref }}}
$$

В формулах (26) использованы следующие обозначения:

$$
F_{x}=F^{\|} \cdot \cos \alpha+F^{\perp} \cdot \sin \alpha, \quad F_{y}=-F^{\|} \cdot \sin \alpha+F^{\perp} \cdot \cos \alpha
$$

- сила лобового сопротивления и подъемная сила, соответственно; $\alpha$ - угол атаки; $S_{\text {ref }}=0.1524$ м $^{2}-$ характерное значение площади, которое для рассматриваемого двумерного случая принималось равным длине хорды; $F^{\|}, F^{\perp}$ - аксиальная и нормальная составляющие полной аэродинамической силы, которые рассчитывались по известному распределению давлений $p$ по поверхности обтекаемого тела

$$
\begin{gathered}
F^{\|}=\int_{L_{i}}\left[-\left(p-p_{\infty}\right) \cdot \cos \left(\vec{n}_{i}, x^{\|}\right)\right] \mathrm{d} L, \\
F^{\perp}=\int_{L_{i}}\left[\left(p-p_{\infty}\right) \cdot \cos \left(\vec{n}_{i}, y^{\perp}\right)\right] \mathrm{d} L
\end{gathered}
$$

Здесь $L_{i}$ - длина $i$-го ребра, аппроксимирующего геометрию крылового профиля; $\vec{n}_{i}-$ нормаль к соответствующему ребру.

На рис. 20 представлены распределения коэффициентов давления на верхней и нижней поверхностях крыла для двух наиболее подробных сеточных моделей. Оба представленных численных решения хорошо соотносятся с экспериментальными данными как качественно, так и количественно. В частности, достаточно точно предсказываются положения двух ударных волн - сильной и слабой, формирующихся, соответственно, на верхней и нижней поверхностях профиля. Однако интенсивность слабого скачка получается несколько заниженной. Чем выше размерность расчетной сетки, тем лучше разрешаются указанные структуры 
течения. Максимальное разрежение на подветренной поверхности крыла, полученное на максимально подробной сетке, соответствует величине $C_{P}=-1.123$, что неплохо согласуется с эмпирическим (эталонным) значением $C_{P}=-1.128$. На наветренной поверхности пиковое падение давления $C_{P}=-0.593$ несколько хуже соотносится с экспериментом $C_{P}=-0.619$. При этом погрешность определения коэффициента подъемной силы не превышает $3 \%$, силы лобового сопротивления - $5 \%$ даже на относительно грубой сетке. В целом, качественное и количественное сопоставление опытных и расчетных данных выявляет допустимое расхождение, которое объясняется пренебрежением важными свойствами реальных физических течений (трехмерностью, вязкостью, теплопроводностью и т.д.).

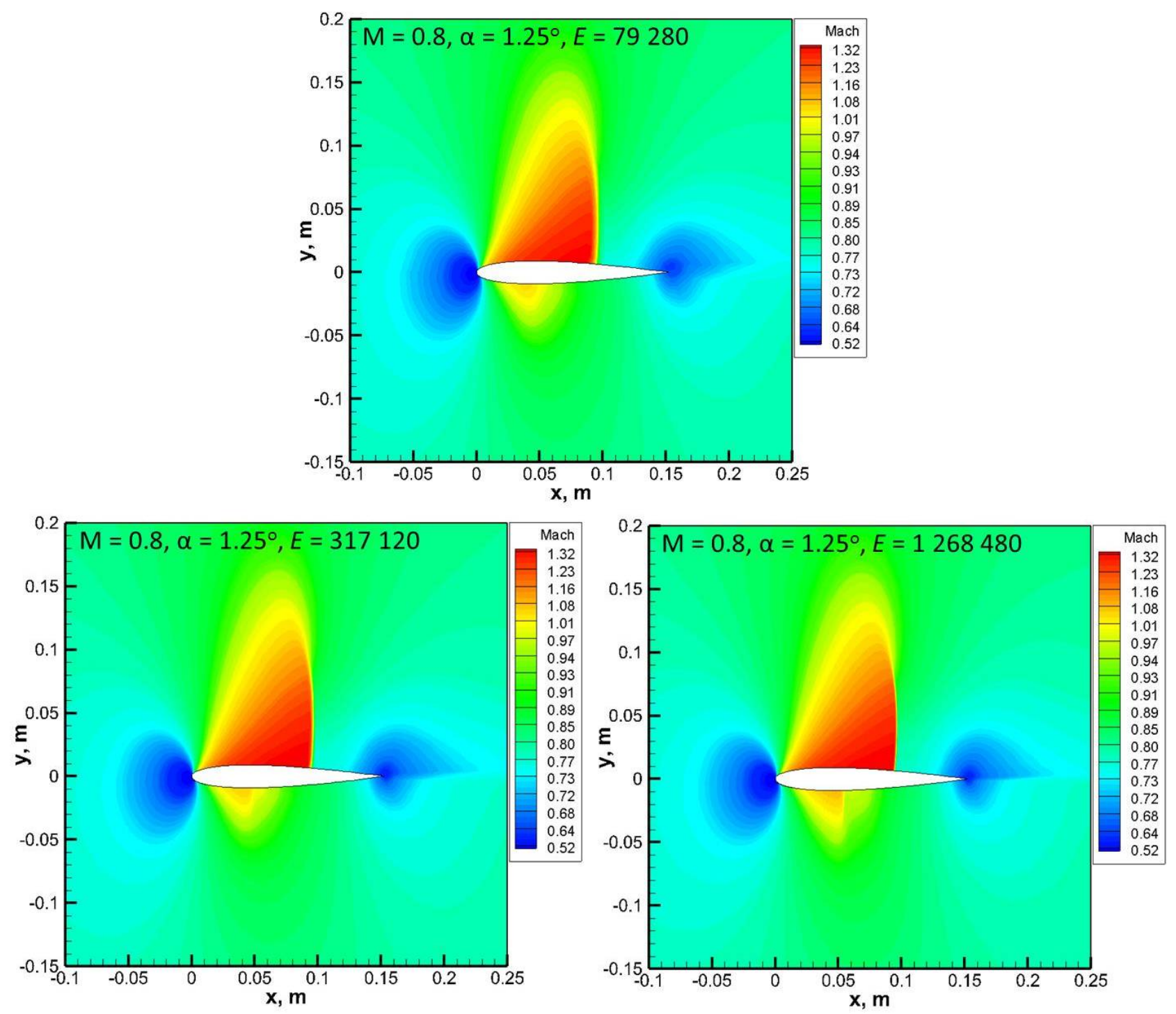

Рис. 19. Расчетная область с сетками различной размерности при последовательном увеличении числа ячеек в четыре раза

\section{2. Заключение}

Работа выполнена на основе анализа обширного пласта англоязычной и отечественной литературы. Анализ публикаций показал, что в литературе, посвященной численным методам решения задач газовой динамики, проблемам верификации и валидации предлагаемых кодов уделяется недостаточно внимания. Большая часть работ носит исследовательский характер и сосредотачивается на таких аспектах моделирования, как вычислительные алгоритмы, построение расчетных сеток, визуализация и т.д. 


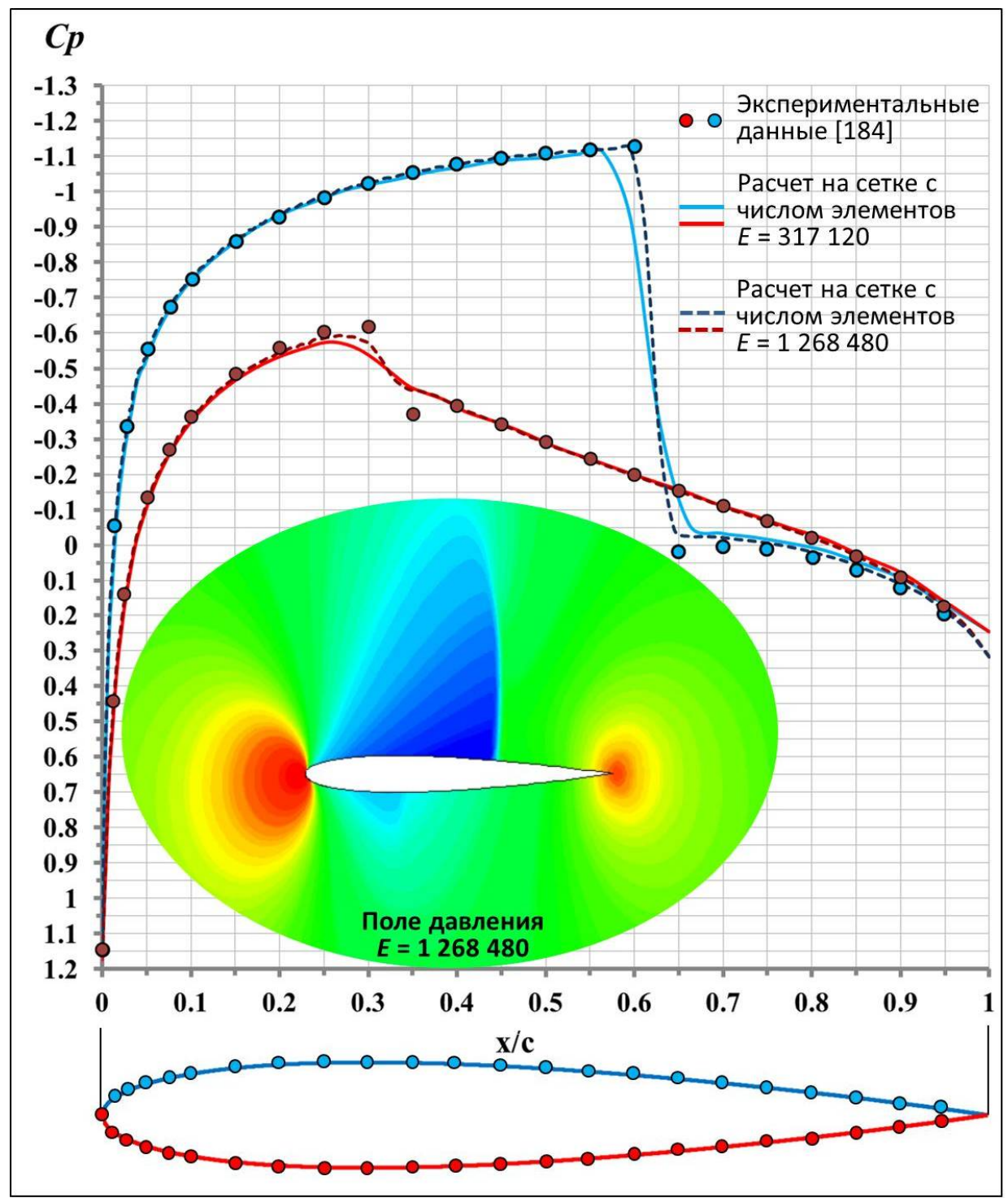

Рис. 20. Сопоставление расчетных профилей коэффициентов давления на поверхности крыла, полученных на наиболее подробных сетках, с экспериментальными данными

Выполненная работа по обобщению многолетнего зарубежного опыта по организации исследований в области верификации и валидации газодинамического ПО может быть полезна для инициации аналогичных отечественных проектов. В задачи последних должно входить: 1) выполнение масштабных расчетно-экспериментальных проектов, задействующих научные, образовательные и производственные организации; 2) проведение специализированных конференций по проблемам технологии верификации и валидации в вычислительной газовой динамике и выпуск специальных серий высокорейтинговых журналов, посвященных данному вопросу; 3) создание общедоступных интернет-ресурсов, содержащих исчерпывающее описание и постановку тестовых задач, а также графические материалы и файлы с расчетными сетками и результатами.

Изложенные технологии верификаии и валидации применены для тестирования двумерной вычислительной модели невязкого течения совершенного газа. Приведено краткое описание концептуальной модели и некоторые детали ее численной реализации в программном коде. Дана постановка тестовых задач, включающая начальные и граничные условия, задание геометрии области моделирования, построение сеточных моделей. Освещены вопросы устойчивости, сеточной и итерационной сходимости. Исследовано влияние аппроксимационной вязкости применяемых численных схем первого и второго порядка точности на 
результаты моделирования. Верификация проводилась на основе сопоставления расчетных данных с аналитическими решениями, доступными для рассматриваемого класса задач. Результаты подтверждают высокую точность генерируемых численных решений.

Для валидации компьютерного кода использовались данные, полученные в ходе натурных испытаний, которые достаточно точно воспроизводят класс плоских ламинарных внешних течений, описываемых реализованной математической моделью. В процессе этих экспериментов обеспечивалась приемлемая точность контроля входных параметров и точность измерения валидационных метрик, которые включали как интегральные, так и распределенные аэродинамические характеристики обтекаемой поверхности профиля NACA0012. Выбранная конфигурация обдуваемого тела, с одной стороны, точно определена, а с другой стороны, не является тривиальной. На основании количественной и качественной оценки расхождения опытных данных с результатами расчетов можно сделать вывод, о возможности ограниченного применения модели для описания узкого круга рассматриваемых реальных физических процессов. Наблюдаемое расхождение объясняется упрощенностью модели и пренебрежением эффектами вязкости, теплопроводности и др.

Несмотря на то, что методологические основы верификации и валидации достаточно хорошо развиты, потребуется затратить значительные усилия на формализацию и оптимизацию процедур корректной количественной оценки адекватности численных реализаций. При этом строгие стандарты верификации и валидации компьютерных кодов газовой динамики только начинают создаваться. Для решения сопряженных задач сложных течений необходимо разрабатывать универсальные эффективные методы количественной оценки точности комплексных вычислительных моделей.

Основные усилия нужно направить на укрепление доверия к вычислительным моделям и уверенности в их прогностических способностях, что может оправдать затраты на верификационную и валидационную деятельность.

Хотя априорная оценка погрешностей вычислений незаменима для широкого класса упрощенных задач, для современных приложений вычислительной газовой динамики возможности данной методики при анализе точности решений очень ограничены. Поэтому основную ставку необходимо сделать на развитие математических методов оценки апостериорных ошибок. Именно на основании известных апостериорных погрешностей можно получить максимально полное представление об эффективности данной численной реализации (компьютерного кода) для конкретной нелинейной системы дифференциальных уравнений в частных производных при определенных начальных и граничных условиях. Наиболее распространенный подход апостериорной оценки погрешности вычислений предполагает использование нескольких численных решений, полученных на ряде последовательно сгущающихся сеток. Учитывая, что при моделировании современных инженерных систем даже однократное сгущение сетки не представляется возможным вследствие ограниченности вычислительных ресурсов, возникает необходимость в разработке методов оценки ошибок на основе результатов, полученных на единственной сетке.

Особое внимание необходимо уделить созданию верификационных и валидационных баз высокоточных данных, а также укреплению кооперации между экспериментаторами, вычислителями, разработчиками кодов и пользователями в развитии технологий верификации и валидации моделей газовой динамики.

Автор выражает благодарность своему научному руководителю, академику РАН, проф. С.Т. Суржикову за идеи, положенные в основу работы.

Работа выполнена в Лаборатории радиационной газовой динамики ИПМех РАН в рамках Программы фундаментальных исследований Российской академии наук, при поддержке гранта РФФИ 16-01-00379. 


\section{Литература}

1. Харитонов А.М. О верификации и валидации моделей и методов численного моделирования пространственных течений // Материалы Международной конференция “Современные проблемы прикладной математики и механики: теория, эксперимент и практика”, Новосибирск, 30 мая - 4 июня, 2011.

2. Железнякова А.Л., Суржиков С.Т. На пути к созданию модели виртуального ГЛА. І. - М.: ИПМех РАН, 2013. - $160 \mathrm{c}$.

3. Wilcox D.C. Formulation of the k-omega Turbulence Model Revisited // AIAA Journal, 2008, V.46, №11, pp. 2823-2838.

4. Wilcox D.C. Turbulence Modeling for CFD. 3rd edition, DCW Industries, Inc., La Canada CA, 2006.

5. Launder B.E., Spalding D.B. The numerical computation of turbulent flows // Computer Methods in Applied Mechanics and Engineering, V.3, №2, 1974, pp. 269-289.

6. Menter F.R. Two-Equation Eddy-Viscosity Turbulence Models for Engineering Applications // AIAA Journal, V.32, №8, August 1994, pp. 1598-1605.

7. Волков К.Н., Емельянов В.Н. Вычислительные технологии в задачах механики жидкости и газа.- М.: Физматлит, 2012. - 468 с.

8. Кулямин В.В. Методы верификации программного обеспечения. - М.: Институт Системного Программирования РАН, 2008. - 111 с.

9. Алексеев А.К., Бондарев А.Е. Применение сопряженных уравнений в задачах верификации и валидации расчетов. - М.: ИПМ им. М. В. Келдыша, 2011. - 15 с.

10. Карпов Ю.Г. Верификация параллельных и распределенных программных систем. - СПб.: БХВ-Петербург, 2010. - 560 с.

11. Вельдер С.Э., Лукин М.А., Шалыто А.А., Яминов Б.Р. Верификация автоматных программ. СПбГУ ИТМО, 2011. - 242 с.

12. Исаев А.И., Скоробогатов С.В. Гидродинамическая верификация и валидация численных методов расчета течения в камере сгорания газотурбинного двигателя // Труды МАИ, 2017, № 97, $26 \mathrm{c}$.

13. Surzhikov S.T. Validation of computational code UST3D by the example of experimental aerodynamic data // Journal of Physics: Conf. Series, 2017, V. 815, 12 p.

14. ГОСТ Р ИСО 9000-2015. Системы менеджмента качества. Основные положения и словарь. Национальный стандарт Российской Федерации.

15. ГОСТ Р ИСО 9001-2015. Системы менеджмента качества. Требования. Национальный стандарт Российской Федерации.

16. ISO 9000:2015. Quality management systems. Fundamentals and vocabulary.

17. ISO 9001:2015. Quality management systems. Requirements.

18. ГОСТ Р 57188-2016. Численное моделирование физических процессов. Термины и определения. 2016, 12 c.

19. ГОСТ Р 57700.12-2018. Численное моделирование сверхзвуковых течений невязкого газа. Верификация ПО / Национальный стандарт РФ по численному моделированию физических процессов. 2018, 20 с.

20. Железнякова А.Л., Суржиков С.Т. Расчет гиперзвукового обтекания тел сложной формы на неструктурированных тетраэдральных сетках с использованием схемы AUSM // TBT, 2014, т. 52, № 2 , с. 283-293.

21. Железнякова А.Л., Суржиков С.Т. Применение метода расщепления по физическим процессам для расчета гиперзвукового обтекания пространственной модели летательного аппарата сложной формы // ТВТ, 2013, том 51, № 6, с. 897-911.

22. Иванов И.Э., Крюков И.А. Метод расчета вязких пространственных течений на нерегулярных сетках // Материалы IX Международной конференции по неравновесным процессам в соплах и струях (NPNJ-2012), Алушта, 25-31 мая, 2012. Изд-во “МАИ-ПРИНТ”, Москва, 2012, с. $240,241$.

23. Ермаков М.К. Многопроцессорное моделирование аэродинамики гиперзвукового летательного аппарата на трехмерных неструктурированных сетках // Материалы XXIII Научно-технической конференции по аэродинамике, п. Володарского Московской обл., 1-2 марта 2012, с. 104,105. 
24. Ермаков М.К. Моделирование гиперзвукового обтекания летательного аппарата на суперкомпьютере “Ломоносов” // 6-я Всероссийская школа-семинар “Аэрофизика и физическая механика классических и квантовых систем”: Сборник научных трудов. - М: ИПМех РАН, 2012, c. $18-23$.

25. Железнякова А.Л., Кузенов В.В., Петрусев А.С., Суржиков С.Т. Расчет аэротермодинамики двух типов моделей спускаемых космических аппаратов. // Физико-химическая кинетика в газовой динамике, 2010, T.9. http://chemphys.edu.ru/media/published/025.pdf

26. Железнякова А.Л., Суржиков С.Т. Численное моделирование гиперзвукового обтекания модели летательного аппарата Х-43 // Вестник МГТУ им. Н.Э. Баумана. - Сер. “Машиностроение”, 2010, №1, c. 3-19.

27. Суржиков С.Т. Аналитические методы построения конечно-разностных сеток для расчета аэротермодинамики спускаемых космических аппаратов // Вестник МГТУ им. Н.Э. Баумана. - Сер. “Машиностроение", 2004, № 2, с. 24-50.

28. Суржиков С.Т. Радиационно-конвективный теплообмен космического аппарата сферической формы в углекислом газе // ТВТ, 2011, Т.49, № 1, с. 92-107.

29. Котов Д.В., Суржиков С.Т. Расчет течений вязкого и невязкого газа на неструктурированных сетках с использованием схемы AUSM // Вычислительная механика сплошных сред, 2011, T.4, №1, c. 36-54.

30. Иванов М.Я., Крайко А.Н. Метод сквозного счета для двумерных и пространственных сверхзвуковых течений // ЖВМ и МФ. 1972, № 3, с. 805-813.

31. Годунов С.К., Забродин А.В., Иванов М.Я., Крайко А.Н., Прокопов Г.П. Численное решение многомерных задач газовой динамики. М.: Наука, 1976. 400 с.

32. Черный Г.Г. Газовая динамика. - М.: Наука. 1988. - 424c.

33. Куликовский А.Г., Погорелов Н.В., Семенов А.Ю. Математические вопросы численного решения гиперболических систем уравнений. М.: Физматлит, 2001. 608 с.

34. Белоцерковский О.М., Давыдов Ю.М. Метод крупных частиц в газовой динамике. - М.: Наука, 1982. - $391 \mathrm{c.}$

35. Крайко А.Н. Теоретическая газовая динамика: классика и современность. - М.: “Торус пресс”, 2010. - 440 c.

36. Popper K.R. The Logic of Scientific Discovery, Basic Books, New York, 1959.

37. Popper K.R. Conjectures and Refutations: The Growth of Scientific Knowledge, Routledge and Kegan, London, 1969.

38. Carnap R. Testability and Meaning // Philosophy of Science, 1963, V.3, pp. 420-468.

39. Kleindorfer G.B., O’Neill L., Ganeshan R. Validation in Simulation: Various Positions in the Philosophy of Science // Management Science, 1998, V.44, №8, pp. 1087-1099.

40. Balci O., Sargent R.G. A Bibliography on the Credibility Assessment and Validation of Simulation and Mathematical Models // Simuletter, 1984, V.15, №3, pp. 15-27.

41. Hamilton M.A. Model Validation: An Annotated Bibliography // Communications in Statistics'-Theory and Methods, 1991, V.20, №7, pp. 2207-2266.

42. Oberkampf W.L. Bibliography for Verification and Validation in Computational Simulation, Sandia National Laboratories, SAND98-2041, Albuquerque, NM, 1998.

43. Вентцель Е.С. Исследование операций: задачи, принципы, методология: учебное пособие. - 5е изд. - М.: КНОРУС, 2013.

44. Barkley D., Henderson R.D. Three-Dimensional Floquet Stability Analysis of the Wake of a Circular Cylinder // Journal of Fluid Mechanics, 1996, V.322, pp. 215-241.

45. Karniadakis G.E., Triantafyllou G.S. Three-Dimensional Dynamics and Transition to Turbulence in the Wake of Bluff Objects // Journal of Fluid Mechanics, 1992, V.238, pp. 1 -30.

46. Verhoff A. Far-Field Computational Boundary Conditions for Three-Dimensional External Flow Problems, AIAA-96-0892 / AIAA 34th Aerospace Sciences Meeting, Reno, NV, 1996.

47. Verhoff A., Cary A. Analytical Euler Solutions for 2D Flows with Corners using Asymptotic Methods, AIAA-98-2687 / 2nd AIAA Theoretical Fluid Mechanics Meeting, Albuquerque, NM, 1998.

48. Roache P.J. Scaling of High-Reynolds-Number Weakly Separated Channel Flows / in Numerical Aspects of Physical Aspects of Aerodynamic Flows, T. Cebecci Ed. Springer-Verlag, New York, 1982, pp. $87-98$.

49. Roache P.J. Need for Control of Numerical Accuracy // Journal of Spacecraft and Rockets, 1990, V.27, №2, pp. 98-102. 
50. Roache P.J. Quantification of Uncertainty in Computational Fluid Dynamics / in Annual Review of Fluid Mechanics, J.L. Lumley and M. Van Dyke Eds. Annual Reviews, Inc., Palo Alto, CA, 1997, pp. 126-160.

51. Roache P.J. Verification and Validation in Computational Science and Engineering, Hermosa Publishers, Albuquerque, NM, 1998.

52. Roache P.J., Knupp P.M. Completed Richardson Extrapolation// Communications in Numerical Methods in Engineering, 1993, V.9, pp. 365-374.

53. Celik I., Hassan Y., Hughes D., Johnson R., Sommerfeld M. Experimental and Computational Aspects of Validation of Multiphase Flow CFD Codes / FED-Vol. 180, The American Society of Mechanical Engineers, United Engineering Center, New York, 1994.

54. Ferziger J.H., Peric M. Further Discussion of Numerical Errors in CFD // International Journal for Numerical Methods in Fluids, 1996, V.23, pp. 1263-1274.

55. Blackwell B.F., Armaly B.F. Computational Aspects of Heat Transfer: Benchmark Problems / ASME HTD-V.258, American Society of Mechanical Engineers, New York, 1993.

56. Blackwell B.F., Pepper D.W. Benchmark Problems for Heat Transfer Codes / ASME HTD-V.222, American Society of Mechanical Engineers, New York, 1992.

57. Mittal R., Balachandar S. Effect of Three-Dimensionality on the Lift and Drag of Nominally TwoDimensional Cylinders // Physics of Fluids, 1995, V.7, №8, pp. 1841-1865.

58. Mittal R., Balachandar S. Vortical Structures in Bluff Body Wakes, AIAA-95-0867 // AIAA 33rd Aerospace Sciences Meeting, Reno, NV, 1995.

59. Blottner F.G. Variable Grid Scheme Applied to Turbulent Boundary Layers // Computer Methods in Applied Mechanics and Engineering, 1974, V.4, pp. 179-194.

60. Blottner F.G. Investigation of Some Finite-Difference Techniques for Solving the Boundary Layer Equations // Computer Methods in Applied Mechanics and Engineering, 1975, V.6, pp. 1-30.

61. Blottner F.G. Accurate Navier-Stokes Results for the Hypersonic Flow over a Spherical Nosetip // Journal of Spacecraft and Rockets, 1990, V.27, №2, pp. 113-122.

62. Shih T.M., Tan C.H., Hwang B.C. Effects of Grid Staggering on Numerical Schemes, International Journal for Numerical Methods in Fluids, 1989, V.9, pp. 193 -212.

63. Zhang X.D., Pelletier D., Trepanier J.Y., Camarero R. Verification of Error Estimators for the Euler Equations, AIAA-2000-1001 / 38th AIAA Aerospace Sciences Meeting, Reno, NV, 2000.

64. Zhang X.D., Trepanier J.Y., Camarero R. An A Posteriori Error Estimation Method Based on Error Equations, AIAA-97-1889 / 13th Computational Fluid Dynamics Conference, Snowmass Village, CO, 1997, pp. 383-397.

65. Ethier C.R., Steinman D.A. Exact Fully 3D Navier-Stokes Solutions for Benchmarking // International Journal for Numerical Methods in Fluids, 1994, V.19, pp. 369 -375.

66. Terrill R.M., Colgan T. Some Simple Analytic Solutions of the Navier-Stokes Equations // International Journal of Engineering Science, 1991, V.29, №1, pp. 55-68.

67. Grinstein F.F. Open Boundary Conditions in the Simulation of Subsonic Turbulent Shear Flows // Journal of Computational Physics, 1994, V.115, pp. $43-55$.

68. Nordstrom J. Accurate Solutions of the Navier-Stokes Equations Despite Unknown Outflow Boundary Data // Journal of Computational Physics, 1994, V.120, pp. 184-205.

69. Boerstoel J.W. Numerical Accuracy Assessment // AGARD-CP-437, Fluid Dynamics Panel Symposium: Validation of Computational Fluid Dynamics, Lisbon, Portugal, 1988.

70. Yoshizawa A. Laminar Viscous Flow Past a Semi-Infinite Flat Plate // Journal of the Physical Society of Japan, 1970, V.28, №3, pp. 776-779.

71. DiMascio A., Paciorri R., Favini B. Convergence of Two Numerical Schemes for Turbulent Boundary Layer Computations, AIAA 98-3009 // 29th AIAA Fluid Dynamics Conference, Albuquerque, NM, 1998.

72. Venkateswaran S., Merkle C.L. Evaluation of Artificial Dissipation Models and Their Relationship to the Accuracy of Euler and Navier-Stokes Computations / Sixteenth International Conference on Numerical Methods in Fluid Dynamics, Arcachon, France, 1998, pp. 427-432.

73. Shih T.M. A Procedure to Debug Computer-Programs // International Journal for Numerical Methods in Engineering, 1985, V.21, №6, pp. 1027-1037.

74. Davis R.T. Laminar Incompressible Flow Past a Semi-Infinite Flat Plate // Journal of Fluid Mechanics, 1967, V.27, №4, pp. $691-704$. 
75. Jameson A., Martinelli L. Mesh Refinement and Modeling Errors in Flow Simulation // AIAA Journal, 1998, V.36, №5, pp. 676 -686.

76. Botta E.F.F., Dijkstra D., Veldman A.E.P. The Numerical Solution of the Navier-Stokes Equations for Laminar, Incompressible Flow Past a Parabolic Cylinder // Journal of Engineering Mathematics, 1972, V.6, №1, pp. 63-81.

77. Shimazaki K., Himeno Y., Baba N. Quantification of Uncertainty in Computational Fluid Dynamics / FED-Vol.-158, Fluids Engineering Conference, Washington, DC, 1993, pp. 19-28.

78. Van De Vooren A.I., Dijkstra D. The Navier-Stokes Solution for Laminar Flow Past a Semi-Infinite Flat Plate // Journal of Engineering Mathematics, 1970, V.4, №1, pp. 9-27.

79. Barragy E., Carey G.F. Stream Function-Vorticity Driven Cavity Solution Using p Finite Elements // Computers and Fluids, 1997, V.26, №5, pp. 453-468.

80. Wang C.Y. Exact Solutions of the Steady-State Navier-Stokes Equations / in Annual Review of Fluid Mechanics, J.L. Lumley and M. Van Dyke Eds. Annual Reviews, Inc., Palo Alto, CA, 1991, p pp. $159-177$.

81. Zingg D.W. Grid Studies for Thin-Layer Navier-Stokes Computations of Airfoil Flowfields // AIAA Journal, 1992, V.30, №10, pp. 2561-2564.

82. AGARD. Quality Assessment for Wind Tunnel Testing, NATO Advisory Group for Aerospace Research \& Development (AGARD), AGARD-AR-304, 1994.

83. AGARD. A Selection of Experimental Test Cases for the Validation of CFD Codes, NATO Advisory Group for Aerospace Research \& Development, AGARD-AR-303-Vol. II, 1994.

84. AGARD. A Selection of Experimental Test Cases for the Validation of CFD Codes, NATO Advisory Group for Aerospace Research \& Development, AGARD-AR-303-Vol. I, 1994.

85. Marvin J.G. Accuracy Requirements and Benchmark Experiments for CFD Validation // AGARD-CP437, Fluid Dynamics Panel Symposium: Validation of Computational Fluid Dynamics, Lisbon, Portugal, 1988.

86. Marvin J.G. CFD Validation Experiments for Hypersonic Flows, AIAA-92-4024 // AIAA 17th Aerospace Ground Testing Conference, Nashville, TN, 1992.

87. Marvin J.G. Perspective on Computational Fluid Dynamics Validation // AIAA Journal, Vol. 33, No. 10, 1995; 1778-1787.

88. Barber T.J. Role of Code Validation and Certification in the Design Environment // AIAA Journal, Vol. 36, No. 5, 1998; 752-758.

89. Oberkampf W.L., Aeschliman D.P. Joint Computational/Experimental Aerodynamics Research on a Hypersonic Vehicle: Part 1, Experimental Results // AIAA Journal, 1992, V.30, №8, pp. 2000 -2009.

90. Oberkampf W.L., Aeschliman D.P., Henfling J.F., Larson D.E. Surface Pressure Measurements for CFD Code Validation in Hypersonic Flow, AIAA Paper 95-2273 // 26th AIAA Fluid Dynamics Conf., San Diego, CA, 1995.

91. Oberkampf W.L., Aeschliman D.P., Henfling J.F., Larson D.E., Payne J.L. Surface Pressure Measurements on a Hypersonic Vehicle, AIAA Paper 96-0669 // 34th Aerospace Sciences Meeting, Reno, NV, 1996.

92. Oberkampf W.L., Blottner F.G. Issues in Computational Fluid Dynamics Code Verification and Validation // AIAA Journal, 1998, V.36, №5, pp. 687-695.

93. Oberkampf W.L., Blottner F.G., Aeschliman D.P. Methodology for Computational Fluid Dynamics Code Verification/Validation, AIAA Paper 95-2226 // 26th AIAA Fluid Dynamics Conf., San Diego, CA, 1995.

94. Bradley R.G. CFD Validation Philosophy // AGARD-CP-437, Fluid Dynamics Panel Symposium: Validation of Computational Fluid Dynamics, Lisbon, Portugal, 1988.

95. Kammeyer M.E. Wind Tunnel Facility Calibrations and Experimental Uncertainty, AIAA 98-2715 // 20th AIAA Advanced Measurement and Ground Testing Technology Conference, Albuquerque, NM, 1998.

96. Mehta U.B. Computational Requirements for Hypersonic Flight Performance Estimates // Journal of Spacecraft and Rockets, 1990, V.27, №2, pp. 103-112.

97. Mehta U.B. Guide to Credible Computational Fluid Dynamics Simulations, AIAA Paper 95-2225 // 26th AIAA Fluid Dynamics Conference, San Diego, CA, 1995.

98. Mehta U.B. Guide to Credible Computer Simulations of Fluid Flows // Journal of Propulsion and Power, 1996, V.12, № 5, pp. 940 - 948. 
99. Deiwert G.S. Issues and Approach to Develop Validated Analysis Tools for Hypersonic Flows: One Perspective / NASA Ames Research Center, NASA-TM-103937, 1992.

100. Van Wie D.M., Rice T. Quantification of Data Uncertainties and Validation of CFD Results in the Development of Hypersonic Airbreathing Engines, AIAA 96-2028 // 27th AIAA Fluid Dynamics Conference, New Orleans, LA, 1996.

101. Cosner R.R. The Role of Validation in the CFD Process at McDonnell Douglas/St. Louis, AIAA-962273 // 19th AIAA Advanced Measurement and Ground Testing Technology Conference, New Orleans, LA, 1996.

102. Cosner R.R. Experimental Data Needs for Risk Management in CFD Applications, AIAA Paper 98$2781 / / 20$ th AIAA Advanced Measurement and Ground Testing Technology Conference, Albuquerque, NM, 1998.

103. Martellucci A. The Challenging Process of Validating CFD Codes, AIAA-90-1402 // AIAA 16th Aerodynamic Ground Testing Conference, Seattle, WA, 1990.

104. Paciorri R., Dieudonne W., Degrez G., Charbonnier J.-M., Deconinck H. Exploring the Validity of the Spalart-Allmaras Turbulence Model for Hypersonic Flows // Journal of Spacecraft and Rockets, 1998, V.35, №2, pp. 121-126.

105. Holden M.S., Moselle J.R., Sweet S.J., Martin S.C. A Database of Aerothermal Measurements in Hypersonic Flow for CFD Validation, AIAA Paper 96-4597 // AIAA 7th International Space Planes and Hypersonic Systems and Technologies Conf., Norfolk, VA, 1996.

106. Gosman A.D. Quality Assurance for Industrial CFD Codes, AIAA 98-2637 // 29th AIAA Fluid Dynamics Conference, Albuquerque, NM, 1998.

107. Springer A.M. Comparison of the Aerodynamic Characteristics of Similar Models in two Different Size Wind Tunnels at Transonic Speeds, AIAA-98-2875 // 20th AIAA Advanced Measurement and Ground Testing Technology Conference, Albuquerque, NM, 1998.

108. Benek J.A., Kraft E.M., Lauer R.F. Validation Issues for Engine - Airframe Integration // AIAA Journal, 1998, V.36, №5, pp. 759-764.

109. Reed H.L., Haynes T.S., Saric W.S. Computational Fluid Dynamics Validation Issues in Transition Modeling // AIAA Journal, 1998, V.36, №5, pp. 742-751.

110. Walker M.A., Oberkampf W.L. Joint Computational/Experimental Aerodynamics Research on a Hypersonic Vehicle: Part 2, Computational Results, AIAA Journal, 1992, V.30, №8, pp. 2010-2016.

111. Aeschliman D.P., Oberkampf W.L. Experimental Methodology for Computational Fluid Dynamics Code Validation / Sandia National Laboratories, SAND95-1189, Albuquerque, NM, 1997.

112. Aeschliman D.P., Oberkampf W.L. Experimental Methodology for Computational Fluid Dynamics Code Validation // AIAA Journal, 1998, V.36, №5, pp. 733-741.

113. Aeschliman D.P., Oberkampf W.L., Blottner F.G. A Proposed Methodology for CFD Code Verification, Calibration, and Validation / Paper 95-CH3482-7, 16th International Congress on Instrumentation for Aerospace Simulation Facilities, Dayton, OH, 1995.

114. Dolling D.S. Problems in the Validation of CFD Codes Through Comparison with Experiment // AGARD-CP-514, Fluid Dynamics Panel Symposium: Theoretical and Experimental Methods in Hypersonic Flows, Torino, Italy, 1992, pp. 1-19.

115. Dolling D.S. High-Speed Turbulent Separated Flows: Consistency of Mathematical Models and Flow Physics // AIAA Journal, 1998, V.36, №5, pp. 725-732.

116. Sindir M.M., Lynch E.D. Overview of the State-of-Practice of Computational Fluid Dynamics in Advanced Propulsion System Design, AIAA Paper 97-2124 // 28th AIAA Fluid Dynamics Conference, Snowmass, CO, 1997.

117. Veazey D.T., Hopf J.C. Comparison of Aerodynamic Data Obtained in the Arnold Engineering Development Center Wind Tunnels 4T and 16T, AIAA 98-2874 // 20th AIAA Advanced Measurement and Ground Testing Technology, Albuquerque, NM, 1998.

118. Coleman H.W., Stern F. Uncertainties and CFD Code Validation // Journal of Fluids Engineering, 1997, V.119, pp. 795-803.

119. Bertin J.J., Martellucci A., Neumann R.D., Stetson K.F. Developing a Data Base for the Calibration and Validation of Hypersonic CFD Codes - Sharp Cones, AIAA Paper 93-3044 // 24th AIAA Fluid Dynamics Conf., Orlando, FL, 1993.

120. Settles G.S., Dodson L.J. Supersonic and Hypersonic Shock/Boundary-Layer Interaction Database // AIAA Journal, 1994, V.32, №7, pp. 1377-1383.

121. Schlesinger S. Terminology for Model Credibility // Simulation, Vol. 32, No. 3, 1979; 103-104. 
122. Guide for the Verification and Validation of Computational Fluid Dynamics Simulations, American Institute of Aeronautics and Astronautics, AIAA-G-077-1998, Reston, VA, 1998.

123. Годунов С.К., Рябенький В.С. Разностные схемы. Введение в теорию. 2-е изд. - М.: Наука, 1977. $-440 \mathrm{c}$.

124. Рябенький В.С., Филиппов А.Ф. Об устойчивости разностных уравнений. - М.: Гостехиздат, 1956. - $172 \mathrm{c}$.

125. Lax P.D., Richtmyer R.D. Survey of the stability of linear finite difference equations // Comm. Pure Appl. Math, 1956. V.9, pp. 267-293.

126. Г.Дж. Майерс. Искусство тестирования программ. - М.: Финансы и статистика, 1982. - 176 с.

127. Г.Дж. Майерс. Надежность программного обеспечения. - М: Мир, 1980. - 359 с.

128. Laney C.B. Computational Gasdynamics, Cambridge University Press, 2007. - 613 p.

129. Yee H.C., Sweby P.K. Aspects of Numerical Uncertainties in Time Marching to Steady-State Numerical Solutions // AIAA Journal, 1998, V.36, №5, pp. 712-724.

130. Ferziger J. H., Peric M. Computational Methods for Fluid Dynamics, 3rd edition, Berlin: Springer, 2002. $-431 \mathrm{p}$.

131. Richtmeyer R.D., Morton K.W. Difference Methods for Initial-Value Problems, Interscience, New York, 1967. $-420 \mathrm{c}$.

132. von Neumann J. Proposal and Analysis of a Numerical Method for the Treatment of Hydrodynamical Shock Problems / Nat. Res. Com. Report AM-551, 1944.

133. Hirsch C. Numerical Computation of Internal and External Flows: Fundamentals of Numerical Discretization, John Wiley, New York, NY, 1988.

134. Hirsch C. Numerical Computation of Internal and External Flows: Computational Methods for Inviscid and Viscous Flows, John Wiley, New York, 1990. - 714 p.

135. Alvin K.F., Oberkampf W.L., Rutherford B.M., Diegert K.V. Methodology for Characterizing Modeling and Discretization Uncertainties in Computational Simulation, Sandia National Laboratories, SAND2000-0515, Albuquerque, NM, 2000.

136. Carpenter M.H., Casper J.H. Accuracy of Shock Capturing in Two Spatial Dimensions // AIAA Journal, 1999, V.37, №9, pp. 1072-1079.

137. Roy C.J., McWherter-Payne M.A., Oberkampf W.L. Verification and Validation for Laminar Hypersonic Flowfields, AIAA 2000-2550, Fluids 2000 Conference, Denver, CO, 2000.

138. de Vahl Davis G. Natural Convection of Air in a Square Cavity: A Benchmark Numerical Solution // International Journal for Numerical Methods in Fluids, 1983, V.3, pp. 249-264.

139. Hatton L. The T Experiments: Errors in Scientific Software // IEEE Computational Science and Engineering, 1997, V.4, №2, pp. 27-38.

140. ГОСТ Р ИСО/МЭК 25001-2017. Информационные технологии (ИТ). Системная и программная инженерия. Требования и оценка качества систем и программного обеспечения (SQuaRE). Планирование и управление. Национальный стандарт Российской Федерации.

141. ГОСТ Р ИСО/МЭК 25010-2015. Информационные технологии (ИТ). Системная и программная инженерия. Требования и оценка качества систем и программного обеспечения (SQuaRE). Moдели качества систем и программных продуктов. Национальный стандарт Российской Федерации.

142. ISO/IEC 25000:2014. Systems and software engineering. Systems and software Quality Requirements and Evaluation (SQuaRE). Guide to SQuaRE.

143. ISO/IEC 25010:2011. Systems and software engineering. Systems and software Quality Requirements and Evaluation (SQuaRE). System and software quality models.

144. Wallace D.R., Ippolito L.M., Cuthill B.B. Reference Information for the Software Verification and Validation Process / Rept. 500-234, 1996.

145. Стандартный глоссарий терминов, используемых в тестировании программного обеспечения. Версия 2.3 (от 9 июля 2014 года) / ред. Erik van Veenendaal, А. Александров. Russian Software Testing Qualifications Board.

146. IEEE 610.12:1990. Standard Glossary of Software Engineering Terminology.

147. IEEE 1028:1997. Standard for Software Reviews and Audits.

148. Роуч П. Вычислительная гидродинамика, 1977. - 606 с.

149. Nishida H., Satofuka N. Higher-Order Solutions of Square Driven Cavity Flow Using a Variable-Order Multi-Grid Method // International Journal for Numerical Methods in Engineering, 1992, V. 34, № 2, pp. 637-653. 
150. Gervais J.J., Lemelin D., Pierre R. Some Experiments with Stability Analysis of Discrete Incompressible Flow in the Lid-Driven Cavity // International Journal for Numerical Methods in Fluids, 1997, V.24, №5, pp. 477-492.

151. Schreiber F., Keller H.B. Driven Cavity Flows by Efficient Numerical Techniques // Journal of Computational Physics, 1983, V.49, №2, pp. 310-333.

152. Guerrero J.S.P., Cotta R.M. Integral Transform Solution for the Lid-Driven Cavity Flow Problem in Streamfunction-Only Formulation // International Journal for Numerical Methods in Fluids, 1992, V.15, №4, pp. 399-409.

153. Hansen E.B., Kelmanson M.A. An Integral Equation Justification of the Boundary Conditions of the Driven-Cavity Problem // Computers and Fluids, 1994, V.23, №1, pp. 225-240.

154. Balachandar S., Mittal R., Najjar F.M. Properties of the Mean Recirculation Region in the Wakes of Two-Dimensional Bluff Bodies // Journal of Fluid Mechanics, 1997, V.351, pp. 167-199.

155. Baber R. The Spine of Software. Designing Provably Correct Software: Theory and Practice, Wiley, New York, 1987. - 316 p.

156. Encyclopedia of Software Engineering / Marciniak J.J. ed. Wiley, New York, 1994. - 1929 p.

157. DeMillo R.A., McCracken W.M., Martin R.J., Passafiume J.F. Software Testing and Evaluation, Benjamin/Cummings, Menlo Park, CA, 1987.

158. Rook P. Software Reliability Handbook, Elsevier Science Publishers, New York, 1990. - 564 p.

159. Dahl O. Verifiable Programming. Prentice-Hall, Englewood Cliff, NJ, 1992.

160. Oberkampf W.L., Aeschliman D.P., Tate R.E., Henfling J.F. Experimental Aerodynamics Research on a Hypersonic Vehicle / Sandia National Laboratories, SAND92-1411, Albuquerque, NM, 1993.

161. Adrian R.J. Scattering particle characteristics and their effect on pulsed laser measurements of fluid flow: speckle velocimetry vs. particle image velocimetry // Applied Optics, 1984, V.23, pp. 1690 1691.

162. Алексеенко С.В., Бильский А.В., Маркович Д.М. Применение метода цифровой трассерной визуализации для анализа турбулентных потоков с периодической составляющей // Приборы и техника эксперимента, 2004, №. 5, с. 145-153.

163. Seitzman J.M., Hanson R.K. Planar Fluorescence Imaging in Gases, Chapter 6 in Instrumentation for Flows with Combustion, ed. A.M.K.P. Taylor, Academic Press, London, 1993.

164. AIAA. Assessment of Experimental Uncertainty With Application to Wind Tunnel Testing, American Institute of Aeronautics and Astronautics, S-071 A-1999, Reston, VA, 1999.

165. Coleman H.W., Steele W.G. Experimentation and Uncertainty Analysis for Engineers / 2nd ed., John Wiley \& Sons, New York, 1999. - 205 p.

166. Box G.E.P., Hunter W.G., Hunter J.S. Statistics for Experimenters: An Introduction to Design, Data Analysis, and Model Building, Wiley, New York, 1978.

167. Cullen A.C., Frey H.C. Probabilistic Techniques in Exposure Assessment: A Handbook for Dealing with Variability and Uncertainty in Models and Inputs, Plenum Press, New York, 1999. - 336 p.

168. Gamerman D. Markov Chain Monte Carlo, Chapman \& Hall, London, 1997. - 342 p.

169. Kleijnen J.P.C. Statistical Tools for Simulation Practitioners, Marcel Dekker, Inc., New York, 1987. $-430 \mathrm{p}$.

170. Mehta U.B. Guide to Credible Computer Simulations of Fluid Flows // Journal of Propulsion and Power, 1996, V.12, №5, pp. 940-948.

171. Cukier R.I., Levine H.B., Shuler K.E. Nonlinear Sensitivity Analysis of Multiparameter Model Systems // Journal of Computational Physics, 1978, V.26, №1, pp. 1-42.

172. Iman R.L., Helton J.C. An Investigation of Uncertainty and Sensitivity Analysis Techniques for Computer Models // Risk Analysis, 1988, V.8, №1, pp. 71-90.

173. Saltelli A., Scott M. The Role of Sensitivity Analysis in the Corroboration of Models and Its Link to Model Structural and Parametric Uncertainty // Reliability Engineering and System Safety, 1997, V.57, pp. $1-4$.

174. Beck M.B. Water Quality Modeling: A Review of the Analysis of Uncertainty // Water Resources Research, 1987, V.23, №8, pp. 1393-1442.

175. Chlond A., Wolkau A. Large-Eddy Simulation of a Nocturnal Stratocumulus-Topped Marine Atmospheric Boundary Layer: An Uncertainty Analysis // Boundary-Layer Meteorology, 2000, V.95, №1, pp. 31-55.

176. Du J., Mullen S.L., Sanders F. Short-Range Ensemble Forecasting of Quantitative Precipitation // Monthly Weather Review, 1997, V.125, №10, pp. 2427-2459. 
177. Palmer T.N. Predicting Uncertainty in Forecasts of Weather and Climate // Reports on Progress in Physics, 2000, V.63, pp.71-116.

178. Lehmann E.L., Romano J.P. Testing Statistical Hypotheses, 3rd edition, Springer, New York, 2005. $-786 \mathrm{p}$.

179. Белоцерковский О.М., Давыдов Ю.М. Метод крупных частиц в газовой динамике. - М.: Наука, 1982. - 391 с.

180. Марчук Г.И. Методы расщепления. - М: Наука, 1988. - 263 с.

181. Liou M. S., Steffen C. A New Flux Splitting Scheme - J. Comput. Phys., Vol. 107, 23-39, 1993.

182. Овсянников Л.В. Лекции по основам газовой динамики. - М.: Ижевск, 2003. -336 с.

183. Крайко А.Н. Краткий курс теоретической газовой динамики. - М.: МФТИ, 2007. - 300 с.

184. Лойцянский Л.Г. Механика жидкости и газа. - М.-Л.: Гостехиздат, 1950. -676 с.

185. Ladson C.L., Hill A.S., Johnson W.G. Pressure Distributions from High Reynolds Number Transonic Tests of an NACA 0012 Airfoil in the Langley 0.3-Meter Transonic Cryogenic Tunnel / NASA TM 100526. $1987.398 \mathrm{p}$.

186. Abbott I.H., von Doenhoff A.E. Theory of wing sections: including a summary of aerofoil data. McGraw-Hill Book Company. 1949. 